\title{
DEVELOPMENT OF MICROFLUIDIC ACOUSTIC FLOW CYTOMETRY BASED ON SIMULTANEOUS ULTRASOUND BACKSCATTER AND PHOTOACOUSTICS FOR MICRON AND SUB-MICRON SIZE OBJECTS
}

\author{
by \\ Vaskar Gnyawali \\ Master of Science in Microsystems Engineering, University of Freiburg, Germany, 2013 \\ Bachelor of Computer Engineering, Tribhuvan University, Nepal, 2009
}

\author{
A dissertation \\ presented to Ryerson University \\ in partial fulfillment of the \\ requirements for the degree of \\ Doctor of Philosophy \\ in the Program of \\ Mechanical Engineering
}

Toronto, Ontario, Canada, 2018

CCVaskar Gnyawali 2018 



\section{AUTHOR'S DECLARATION FOR ELECTRONIC SUBMISSION OF A DISSERTATION}

I hereby declare that I am the sole author of this dissertation. This is a true copy of the thesis, including any required final revisions, as accepted by my examiners.

I authorize Ryerson University to lend this dissertation to other institutions or individuals for the purpose of scholarly research.

I further authorize Ryerson University to reproduce this dissertation by photocopying or by other means, in total or in part, at the request of other institutions or individuals for the purpose of scholarly research.

I understand that my dissertation may be made electronically available to the public. 

Development of microfluidic acoustic flow cytometry based on simultaneous ultrasound backscatter and photoacoustics for micron and sub-micron size objects

Doctor of Philosophy 2018

Vaskar Gnyawali

Mechanical Engineering

Ryerson University

\begin{abstract}
I developed a flow cytometer based on simultaneous detection of ultra-high frequency ultrasound backscatter and photoacoustic waves from individual micron scale objects, such as, cells, microparticles, and microbubbles flowing in a microfluidic channel.
\end{abstract}

Individual micron scale objects are flow focused through a focal zone, where both ultrasound and laser pulses focus, in a microchannel of a polydimethylsiloxane (PDMS) based microfluidic device. At the focal zone, the objects are simultaneously insonified by ultrasound (center frequency $375 \mathrm{MHz}$ ) and irradiated by nanosecond laser (532 nm wavelength) pulses. The interactions generate ultrasound backscatter and photoacoustic signals from the individual objects, which are strongly dependent on their size, morphology, and biomechanical properties, such as the Young's modulus, and optical absorption properties. These parameters can be extracted by analyzing the unique spectral features of the detected signals. At frequencies less than $100 \mathrm{MHz}$, the signals from the micron scale objects do not contain these unique spectral signatures, thus higher frequencies are required.

Cell analysis is the main application of interest using the acoustic flow cytometer. Combining ultrasound backscatter and photoacoustics results in sufficient information about a single cell that can be used for single cell analysis and for diagnostics applications. However, the usage of this system is not 
limited to biological cells. This system can also be used for analyzing individual microbubbles, which are used as ultrasound contrast agents. During my research, a novel microfluidic technique is developed to generate microbubbles of desired sizes by shrinking microbubbles from $O(100) \mu \mathrm{m}$ by applying a suitable vacuum pressure. These shrunken bubbles of different sizes can be used as samples to validate the acoustic flow system for microbubble analysis.

The flow cytometry technique presented in this thesis can be used to identify and count label-free red blood cells (RBCs) and white blood cells (WBCs) in a sample of blood. The results from acoustic flow cytometer agree with the results obtained from the fluorescence-tagged samples in a fluorescenceactivated cell sorting (FACS) system. I anticipate that this novel label-free technique will allow for a rapid analysis of individual label-free cells for diagnostic applications. 


\section{Acknowledgements}

Firstly, I would like to express my sincere gratitude to my supervisors, Dr. Scott Tsai, Department of Mechanical and Industrial Engineering, and Dr. Michael Kolios, Department of Physics, Ryerson University, Canada for providing close supervision, expert guidance, support and patience throughout my study. Their encouragement helped me to explore myself and shape my abilities as a researcher.

My sincere thanks to my examining committee chair Dr. Ahmad Ghasempoor, and members Drs. Marcello Papini and Ziad Saghir.

Also, I would like to thank Dr. Eric Strohm for his suggestions and help as a colleague and for his expert advice regarding experiments, knowledge, and manuscript correction. I am very thankful to all the past and present members of the LoFFI and the Kolios lab for making my four years a fun, memorable and enjoyable. I would like to remember Dr. Byeong-Ui Moon, Steven jones, Brian Battaglia, Nevetha Yogarajha, Niki Abbasi, Morteza Jeyhani, Huma Inayat, Maryam Navi, Stephanie Buryk, Mohhamadali Saremi, Jennifer Keida, Ali Salari and Jiang Xu from LoffI. Similarly, Dr. Krishnan Sathiyamoorthy, Michael Moore, Yanjie Wang, and Dr. Christopher Smeenk from the Kolios Lab. Many of the discussions with them have directly and indirectly contributed to this research. In particular, I would like to acknowledge Devin Ostrom (technical officer, Mechanical Engineering department) for his help in CNC

machining and 3D printing the molds, Elizabeth Berndl for extensive biological related help, Arthur Worthington, and Kevin Liu.

My cordial Acknowledgement goes to my family: my parents, wife, sister, brother-in-law, uncles, aunts, cousin brothers and sisters, and in-laws for their continuous support, love, and faith in me. 



\section{Dedication}

To my loving family and friends 



\section{Contents}

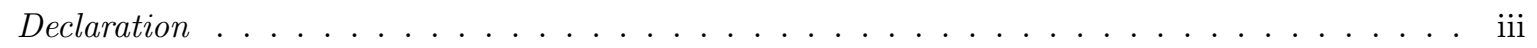

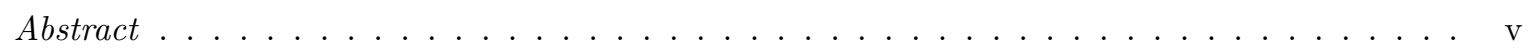

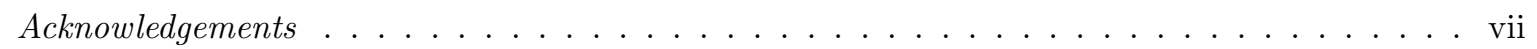

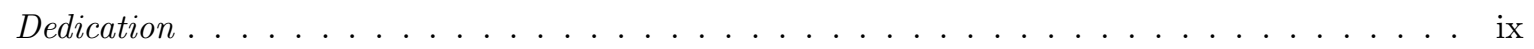

List of Tables . . . . . . . . . . . . . . . . . . . . . . . . xiii

List of Figures . . . . . . . . . . . . . . . . . . . . . . xv

\begin{tabular}{lll}
\hline & Introduction & 1
\end{tabular}

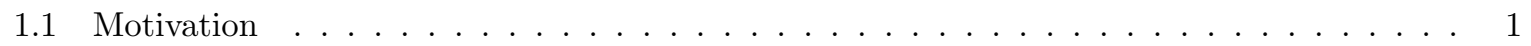

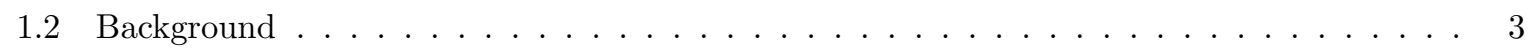

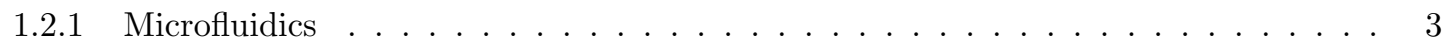

1.2 .2 Ultrasound and photoacoustics $\ldots \ldots \ldots \ldots \ldots \ldots \ldots$

1.2 .3 Microbubbles . . . . . . . . . . . . . . . . . . . . . . 9

1.3 Objective and hypothesis $\ldots \ldots \ldots \ldots \ldots \ldots \ldots \ldots \ldots$

1.4 Overview of the dissertation $\ldots \ldots \ldots \ldots \ldots \ldots \ldots$

1.5 Author's contribution in the context of collaboration . . . . . . . . . . . . . . . 12

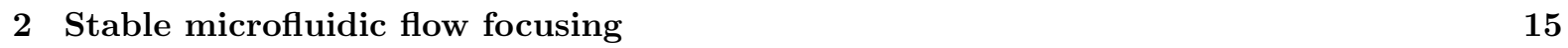

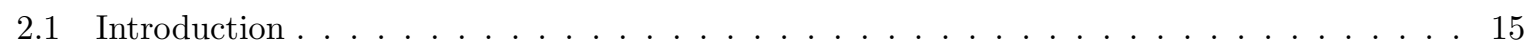

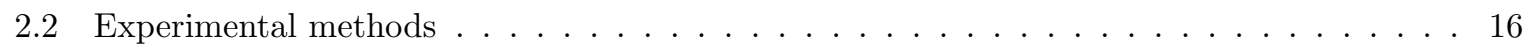

2.2 .1 Device fabrication $\ldots \ldots \ldots \ldots \ldots \ldots \ldots \ldots$

$2.2 .2 \quad$ Experimental setup $\ldots \ldots \ldots \ldots \ldots \ldots \ldots \ldots$

2.3 Result and discussion $\ldots \ldots \ldots \ldots \ldots$

2.3 .1 The stability of hydrostatics and syringe pump based flow focusing . . . . . . . 19

2.3 .2 Controlling hydrostatics based flow focusing . . . . . . . . . . . . . 22

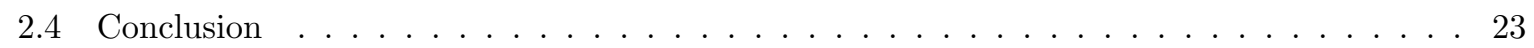

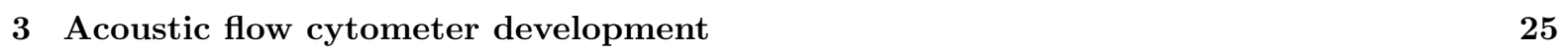

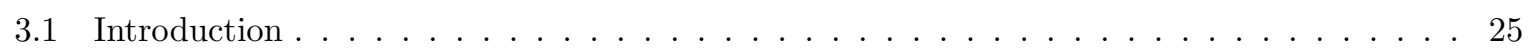

3.2 Methods . . . . . . . . . . . . . . . . . . . . . . . . . . . . . . 28

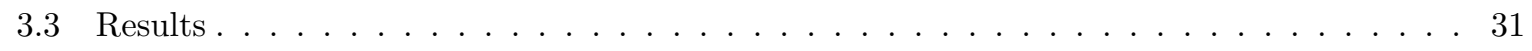


$3.3 .1 \quad$ Acoustic flow cytometry concept . . . . . . . . . . . . . . . . . . . 31

$3.3 .2 \quad$ Generation of ultrasound backscatter and photoacoustic signals . . . . . . . . . . 32

$3.3 .3 \quad$ Calibration of the acoustic flow system $\ldots \ldots \ldots \ldots \ldots \ldots$

$3.3 .4 \quad$ Comparison with Fluorescence Activated Cell Sorting (FACS) . . . . . . . . . . . . 34

3.3 .5 Label-free blood cell differentiation . . . . . . . . . . . . . . . . 36

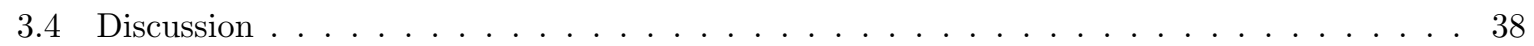

3.5 Conclusion $\ldots \ldots \ldots \ldots \ldots \ldots$

\begin{tabular}{|lll}
4 & Microfluidic generation and shrinkage of microbubbles using vacuum pressure & 41
\end{tabular}

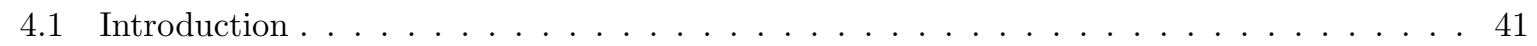

4.2 Methods . . . . . . . . . . . . . . . . . . . . . . . . . . . . . . . . . . . . . 43

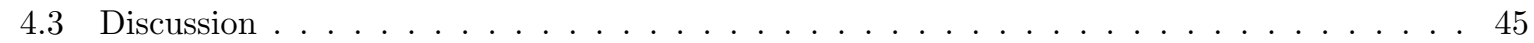

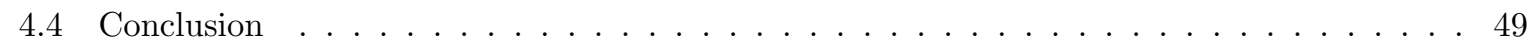

$\begin{array}{lll}5 & \text { Conclusions and future work } & 51\end{array}$

5.1 Summary of findings $\ldots \ldots \ldots \ldots \ldots \ldots \ldots \ldots \ldots$

$5.1 .1 \quad$ Stable flow focusing using microfluidics (Chapter 2) . . . . . . . . . . . . . 51

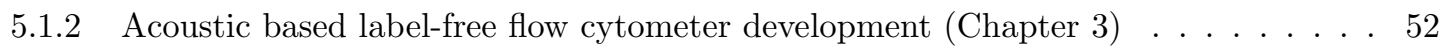

$5.1 .3 \quad$ Microfluidic microbubble shrinking system (Chapter 4$) \quad \ldots \ldots \ldots$. . . . . 52

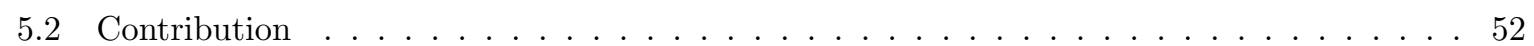

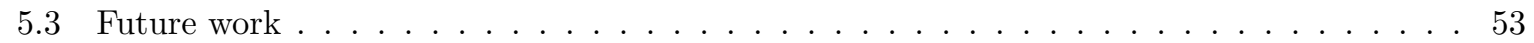

\begin{tabular}{|l|l|}
\hline A Supplementary information for chapter 2 & 55
\end{tabular}

\begin{tabular}{|l|l}
\hline B Supplementary information for chapter 3 & 57
\end{tabular}

\begin{tabular}{|l|l|}
\hline C Supplementary information for chapter 4 & 61
\end{tabular}

\begin{tabular}{ll}
\hline D Future work & 65
\end{tabular}

D.1 Measuring nucleus-to-cytoplasmic (N:C) ratio of single cells in a population . . . . . . . 65

D.2 Characterization of microbubbles and nanobubbles $\ldots \ldots \ldots \ldots$. . . . . . . 68

\begin{tabular}{|ll}
\hline E List of publications & 73
\end{tabular}

\begin{tabular}{ll}
\hline References & 87
\end{tabular} 


\section{List of Tables}

D.1 Average amplitude of the signals detected by the flow cytometer for different average size of nanobubbles measured by the Archimedes ${ }^{\circledR}$ sizer. . . . . . . . . . . . . . . . . 70 



\section{List of Figures}

1.1 Hydrodynamic flow focusing in microfluidic devices. a) Schematic of a two-dimensional flow focusing design where flow from the inlet is focused in lateral directions by the sheath flows from the side channels. In the figure, $W_{f}$ is the width of focused flow and $W_{c}$ is the width of the focusing channel adopted from (25). b) A three-dimensional flow-focusing design. The top figure shows the top view of the design adopted from (26). Inlets $A, C$, and $E$ are for sheath flows and $B$ is for the sample flow and the height of the channels are color coded. The middle figure shows the segment of the design where the flows meet. The lower figure shows the design in three dimensions with red indicating the sample flow being focused downstream of the microchannel. . . . . . . . . . . . . . . . 5

1.2 Ultrasound imaging resolution as a function of the ultrasound wave frequency. (Courtesy

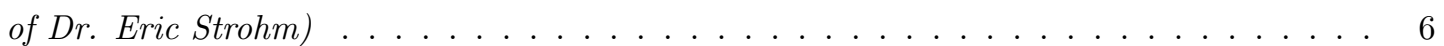

$1.3 \quad$ Schematic illustration of the generation of ultrasound backscatter and photoacoustic waves from a spherical particle. a) Ultrasound: the particle scatters the incident pressure waves, which are detected by the probing transducer as backscatter. b) Photoacoustics: a particle irradiated by a laser pulse absorbs the optical energy and undergoes thermal expansion to generate photoacoustic waves. The ultrasound transducer can also be used to detect the photoacoustic waves. . . . . . . . . . . . . . . . . . . . . . . . . . . . . 8

1.4 Frequency spectra of the ultrasound backscatter and photoacoustic signals from individual fluid spheres of varying diameters: a) $2.5 \mu \mathrm{m}$ b) $5 \mu \mathrm{m}$ and c) $10 \mu \mathrm{m}$. The spacing between the minima is related to the size of the sphere. . . . . . . . . . . . . . . . . 9

$1.5 \quad$ Schematic illustration of a microbubble with a gas core and lipid shell suspended in a liquid

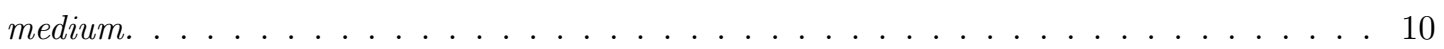


2.1 The hydrostatics-based microfluidic device. Schematic illustrations show that (a) fluid flow is driven by hydrostatic pressure that is controlled by the liquid column heights $H_{1}$ and $H_{2}$ of the sample (red) and sheath (blue) liquids, respectively. The sample flow is supplied to the microchannel via a needle that is inserted to the side of the PDMS slab and the sheath flow enters the microchannel via tubing connected from the top of the device. (b) Extended isometric section view of the flow focusing cross-junction shows that the sheath flow surrounds and flow focuses the sample flow. Sample and sheath fluids both flow in the direction indicated by the $x$-axis. (c) Cross section view of the microchannel downstream of the cross-junction shows the diameter, $d_{s}$, of the flow of the sample fluid. Experimental image showing (d) the flow focusing of the sample fluid near the cross junction. Scale bar represents $50 \mu \mathrm{m} . \ldots \ldots \ldots \ldots \ldots$. . . . . . . . . 17

2.2 Experimental images of the sample fluid (black) focused inside a microchannel downstream of the focusing junction. These images are taken from video frames of the experiments whose focused flow average diameter, $d_{s}=59 \mu \mathrm{m}$, and the frames are selected at an interval of $50 \mathrm{~ms}$. Brightfield images of the focused flows controlled by (a) hydrostatic pressure and (b) constant flow-rate syringe pumps. Thresholded images of the same focused flows produced with (c) hydrostatic pressure and (d) constant flow-rate syringe pumps. The image sequences show qualitatively that the hydrostatics-controlled focused flow has a more consistent diameter, $d_{s}$, than syringe pump driven flow. Syringe-pump driven focused flows also exhibit more erratic flow behavior. Flow is in the direction indicated by the $x$-axis. Scale bars represent $50 \mu \mathrm{m}$. . . . . . . . . . . . . . 19

$2.3 \quad$ A plot of the focused flow diameter, $d_{s}$, versus time, $t$, for experiments using hydrostatic pressure (blue triangle) and syringe pumps (red diamond) over a period $\Delta t=0.7 \mathrm{~s}$. The average diameter, $d_{s}$, of the focused flow in both hydrostatics and syringe-pump experiments is $d_{s}=59 \mu \mathrm{m}$ (indicated by the black line). $t=0 \mathrm{~s}$ represents the first frame of the high speed video. The data shows significantly larger fluctuations in the syringe

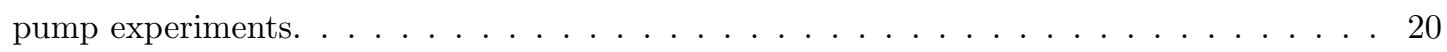

2.4 A plot of the cumulative normal distribution function versus dimensionless focused flow diameter, $d_{s} / w$, in the microfluidic channel driven by hydrostatic pressure (blue dashed lines) and syringe pumps (red solid lines). The four sets of data correspond to averaged focused flow widths, $d_{s} / w=0.075,0.100,0.140$ and 0.197 (from left to right). Hydrostaticscontrolled flows show consistently narrower distributions of the focused width, $d_{s} / w$, compared to the focused flow generated by syringe pumps. . . . . . . . . . . . . . . 21 
$2.5 \quad$ A plot of the dimensionless focused sample flow diameter, $d_{s} / w$, versus the ratio of liquid column heights, $\frac{H_{1}}{H_{1}+H_{2}}$. Here, the data shows results from six experiments where the sample liquid column height, $H_{1}=84,89,90,100,105$, and $113 \mathrm{~mm}$, and the sheath flow liquid column height, $\mathrm{H}_{2}$, is varied to achieve different focused sample flow diameters, $d_{s} / w$. The results indicate monotonic and approximately proportional increase of the focused sample flow diameter, $d_{s} / w$, with the ratio of liquid column heights, $\frac{H_{1}}{H_{1}+H_{2}}$, suggesting that the parameter $\frac{H_{1}}{H_{1}+H_{2}}$ is a good controller for the degree of hydrostatics

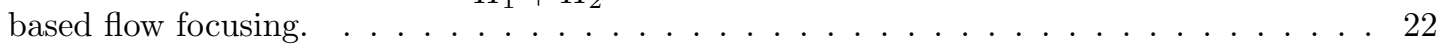

3.1 Conceptual schematic illustration of the acoustic flow cytometry system. a) The illustra-

\begin{tabular}{|c|c|c|c|c|c|c|}
\hline tion shows the overall design of a microfluidic device with collinearly aligned ultrasound \\
\hline transducer and laser focusing optical objective. b) Hydrodynamic 3D flow focusing of a
\end{tabular} sample flow in the microfluidic device. The isometric view shows how the sample flow infused through the embedded needle at the cross-junction is 3D focused by the sheath

flows from the side channels. The top and side views illustrate the position of the needle relative to the channel geometry. Gaps above, below, and on the sides of the needle allow

the sheath flows to push the sample flow to the center of the channel. c) The magnified view of a collinearly aligned transducer and laser beam. The incident ultrasound (US) wave and laser beam interact with a particle at the interrogation zone producing both ultrasound backscatter and photoacoustic (PA) waves. d) Image of an experimental setup that shows how the laser is focused on the interrogation zone of the ultrasound transducer. 30

3.2 (a) the ultrasound (US) backscatter and (b) the photoacoustic (PA) waves that are generated by a particle located in the focal zone of the transducer, as a result of the ultrasound and laser pulses that interact with the particle, respectively. . . . . . . . . . . . . 32

3.3 (a) A representative RF line detected and recorded by the ultrasound (US) transducer from a $3 \mu \mathrm{m}$ diameter black polystyrene particle. The ultrasound and photoacoustic (PA) signals in the RF line are highlighted in the red boxes. Transducer artifacts are removed from the signal to emphasize the detected ultrasound backscatter and photoacoustic waves. Measured ultrasound backscatter (c) and photoacoustic (b) signals from the individual particle. These signals are gated using hamming window from the RF line. . . . . . . . . 32

3.4 Maximum amplitude plot of the time domain signals of the ultrasound (US) backscatter and photoacoustic (PA) waves detected by the transducer for three different samples: black particles exclusively (top row), white particles exclusively (middle row), and a mixture of both types of particles (bottom row). The $y$-axes represent 50 particles detected by the acoustic flow cytometer from each experiment. The $x$-axes represent the "slow-time" of the acquisition by the transducer while the particles flow downstream. The amplitude of the detected signals (in mv) is represented by color bars. . . . . . . . . . . . . 33 
$3.5 \quad$ A comparison plot of the percentage of black particles in sample solutions of a mixture of black and white particles detected by the acoustic flow cytometer and the FACS. Results from the acoustic flow cytometer are comparable with those from the FACS. Error bars represent one standard deviation with data from five independent experiments. The straight line is the line of equality for both techniques. . . . . . . . . . . . 35

3.6 Representative signals detected by the transducer for a) RBC and b) WBC samples. The signal from an RBC contains both ultrasound (US) backscatter and photoacoustic (PA) waves whereas signals from WBC contains only ultrasound backscatter. . . . . . . . . . 36

3.7 Plot showing the percentage of RBCs in mixtures of RBCs and WBCs from five sample solutions containing WBCs and RBCs using the acoustic flow cytometer (labeled AFC) and the FACS. The $y$-axis represents the percentage of the RBCs present in the sample solutions. For each experiment, the sample solutions are divided to three different volumes. The first volume is label-free, the second is incubated with DRAQ5 fluorescent dye, and the third is incubated with R-PE conjugated CD45. DRAQ5 stains WBC nuclei and CD45 bonds to the WBC membrane. The label-free solution is measured using the acoustic flow cytometer, and the other two are measured using the FACS. Error bars represent one standard deviation from the mean for three experiments. . . . . . . . . . . . 37

4.1 Our microfluidic device for microbubble generation and shrinking. (a) The three dimensional (3D) schematic diagram shows that bubbles are (b) generated at the orifice and (c-f) shrink as they flow downstream in the serpentine microchannel. Two sets of vacuum channels are embedded adjacent to the serpentine channel. Vacuum inlets 1 and 2 connect the vacuum source to the vacuum microchannels. Representative experimental images (b - f) show the sequential shrinking of the microbubbles in the microchannel. These experimental images are taken from an experiment where the vacuum pressure, $P_{v}=-50 \mathrm{kPa}$.

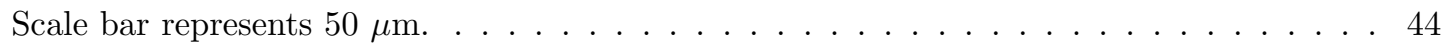

4.2 A plot of the normalized final microbubble volume $\left(V_{f} / V_{i}\right)$ versus the applied vacuum channel pressure, $P_{v}$, in the vacuum microchannels. These experimental results show that, even in the absence of an applied vacuum pressure, the microbubbles shrink to approximately $55 \%$ of their initial volume when flowing through the long serpentine channel. When a vacuum pressure is applied to the vacuum microchannels, we observe that the microbubbles in the serpentine microchannel shrink more dramatically, and their shrinkage increases monotonically with increasing magnitude of the applied vacuum pressure. Here, error bars represent one standard deviation of 10 samples. The insets show representative experimental images of microbubbles at the initial and final positions in the serpentine microchannel, corresponding to microbubble volumes $V_{i}$ and $V_{f}$, respectively. The datum indicated by "*" corresponds to the final microbubble volume $V_{f} / V_{i}$ from the experiment where the vacuum pressure is $P_{v}=-90 \mathrm{kPa}$. Here, the microbubbles are not visible under the microscope, so we define their volume $V_{f} / V_{i}=0$. The inset images are from an experiment where the vacuum pressure $P_{v}=-50 \mathrm{kPa}$. Scale bars represent $50 \mu \mathrm{m}$. 46 
4.3 Representative sequential images of microbubbles collected at the outlet of the microfluidic device. (a) Microbubbles in the control experiment where the applied vacuum pressure, $P_{v}=0 \mathrm{kPa}$, observed via a 10x objective. (b) Microbubbles generated by a vacuum pressure, $P_{v}=-70 \mathrm{kPa}$, observed with a $63 \mathrm{x}$ objective. Here, arrows indicate the position of representative microbubbles in the sample. The microbubble shown in each frame may not be the same due to difficulties tracking the moving bubbles, but all microbubbles had diameter, $D_{f}=1-7 \mu \mathrm{m}$. Image frames are at 5 minute intervals for both (a) and (b). Scale bars represent $50 \mu \mathrm{m} . \ldots \ldots \ldots \ldots \ldots \ldots \ldots$

4.4 A plot of the diameter $D_{f}$ of the collected microbubbles versus time $t$. The data shown are from three representative experiments using vacuum pressures of $P_{v}=0,-60$, and -70

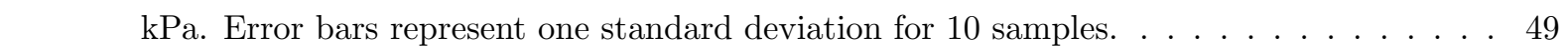

A.1 A plot of an average focused flow diameter, $d_{s}$, versus total experimental time, $t$, for an experiment using hydrostatics over a period of 5 min. $\ldots \ldots \ldots \ldots 6$

B.1 The schematic illustration of slow and fast-time axes representing signals detected by the US transducer while a particle flows through the focal zone of the transducer. . . . . . . 57

B.2 $\quad$ FACS results from $3 \mu \mathrm{m}$ diameter polystyrene black and white particles, which are denoted as black and white, respectively. Data detected in the APC and BV605 channels (a) and PE-Cy7 and PE Cy5 channels (b) for the same sample of particles. . . . . . . . . . 58

B.3 Components and steps used to fabricate the microfluidic device. a) The US transducer; b) the 3D printed transducer mold; c) the 3D printed alignment frame; d) the CNC machined metal mold. (e) The transducer mold replaced by the US transducer in the final microfluidic device. $\mathrm{f}$ ) The backside view of the device. . . . . . . . . . . . . . 59

B.4 Flow diagram of the acoustic flow cytometer. Arrows indicate direction of travel of either signals, fluids, or light depending on the module. . . . . . . . . . . . . . . 59

C.1 The schematic diagram of the microbubble shrinkage system. We supply air through a pressure valve where we fix the inlet air pressure to $P_{a}=4$ psi for all the experiments.

Simultaneously, we infuse the lipid-mixture using a syringe pump. The infuse flow rate is also fixed at $\mathrm{Q}=4 \mu \mathrm{L} / \mathrm{min}$ for all the experiments. Our only tuned variable, vacuum pressure, $P_{v}$, is supplied through two inlets using a Mityvac vacuum pump, which consists of an integrated control valve. The bubble is generated at the flow focusing orifice between air and lipid mixture channels, and flows downstream through the serpentine channel where the microbubbles shrink due to the effect of vacuum in the adjacent interdigitated vacuum channels. The schematic diagram is not in scale. . . . . . . . . . . . . 62

C.2 Schematic representation of different shapes of microbubbles. (a) Spherical microbubble, (b) spherical cap of a microbubble, and (c) a discoid microbubble. . . . . . . . . . . . 62 
D.1 a) A representative time-gated time domain ultrasound backscatter signal and frequency domain ultrasound backscatter and (b) photoacoustic signal detected from an MCF7 cell. The portion of the signal outside the bandwidth of the transducer is shaded grey in the figure. 66

D.2 Histograms showing a distribution of the estimated size of (a) cells and (b) nuclei. The size of the cells and their nuclei are estimated using the ultrasound backscatter and the photoacoustic signals detected from the acoustic flow cytometer, respectively. . . . . . . . . 67

D.3 The average amplitude of the ultrasound backscatter signals detected from the individual shrunken micro/nanobubbles at different vacuum pressures. Error bars represent one standard deviation from at least 20 microbubbles for each vacuum pressure. . . . . . . . . . 69

D.4 Histograms of the amplitudes of the ultrasound backscatter detected by the acoustic flow cytometer from individual scatterers in the nanobubble solution. These signals were detected from the nanobubbles whose average size, as measured by A are $150 \mathrm{~nm}$ (a), 225 $n m(b)$, and $350 \mathrm{~nm}(\mathrm{c})$ when measured by the Archimedes ${ }^{\mathrm{A}}$ sizer. . . . . . . . . . . . . . 70

D.5 Histograms of the amplitudes of the ultrasound backscatter and photoacoustic signals simultaneously detected from individual black lipid nanobubbles (dyed using Sudan Black) using the acoustic flow cytometer. These signals were detected from the nanobubbles whose

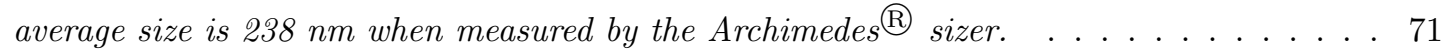




\section{Chapter 1}

\section{Introduction}

\subsection{Motivation}

Flow cytometry is a commonly used technique in many clinical and biomedical laboratories for high throughput experimentation of single cells in a population.(1; 2) It is a crucial instrument for clinical diagnostics and hematology-related assays and is widely used in molecular biology, pathology, immunology, plant biology, and marine biology.(1, 3) Conventional flow cytometers are based on an optical approach, which uses scattering and fluorescence emission to provide information on size, granularity, and the expression level of chromophores bound on the cell surface and/or enclosed cell components. As an alternative to the optical technique, an electrical impedance based flow cytometer detects a change in electrical impedance of the individual cells to provide their physical information.(4, 5)

Current optical systems are fast and precise in the screening and sorting of cells, but have inherent drawbacks. First, these systems usually require sample preprocessing using fluorescent biomarkers and antibodies which might alter the system that is under investigation. (1, 6) Second, the quality of the fluorescence data depends on the quality of the binding dynamics.(7) Lastly, the equipment required to run these systems are expensive and complex to operate. (1; 6) On the other hand, alternative systems such as impedance-based systems are less sensitive, have minimal chemical specificity, and are limited to counting and sizing of single cells. $(1 ; 8,9)$

In reviewing the current systems, it is clear that there is an unmet need for a label-free flow cytometry system that is capable of analyzing individual cells. The goal of this research is to develop an acoustic flow cytometer which is an alternative, label-free, flow cytometer that is based on ultrasound backscatter and photoacoustics, which are simultaneously generated from individual cells. This approach requires ultrasound and photoacoustics at ultra-high frequencies (UHF) measuring over $100 \mathrm{MHz}$. At these high frequencies, the wavelength is in the size range of a single cell. The ultrasound backscatter signals 
detected from an individual cell in the flow cytometer provides information on the bulk properties of that cell, such as biomechanical properties. The photoacoustic signals provide information on optical absorption, which relates to the chromophore composition of the cells. (10) Notably, the multi-parametric analysis of the individual cells, using both ultrasound backscatter and photoacoustics, provides more information from a single experiment than existing systems. The additional information acquired from the multi-parametric analysis of individual cells within a cell population can be correlated with pathological conditions specific to the source cell population.

A potential application of the acoustic flow cytometer, beyond that of biological cells, is to analyze individual microbubbles. Microbubbles are volumes of gas enclosed in a lipid shell measuring hundreds of micrometers in diameter. Microbubbles are used as contrast agents for ultrasound imaging diagnostic applications, which require the microbubbles of 1-7 $\mu \mathrm{m}$ in diameter. 11 , 12, 13) Monodisperse microbubbles are important for some clinical applications. However, microbubbles generated by classical techniques, including commercially available techniques, are polydisperse.(14) Microfluidic based microbubble generation is a recent technique that allows for the generation of monodisperse microbubbles. 15,16 ; 17) However, existing microfluidic techniques require a complicated manufacturing process due to the size of the orifice needed to generate relevant sized microbubbles.(15) The microfluidic system presented in this thesis produces the relevant sized microbubbles $(1-7 \mu \mathrm{m}$ in diameter) by combining a simple microfluidic device manufacturing technique with integrated vacuum pressure.

To measure the diameter of the microbubbles, existing techniques use either ultrasound signals with frequencies up to $25 \mathrm{MHz}$ or an optical imaging technique, both of which use a limited number of sample microbubbles. $(14,18,19)$ Similarly, a recent resonant mass measurement based device, Archimedes ${ }^{\circledR},(20)$ measures the size of the microbubbles and nanobubbles. Experiments show that measurements are limited to small sized bubbles. It has been shown that spatial resolution of the ultrasound increases with ultrasound frequency. Therefore, using ultra-high frequency can be beneficial to analyze these microbubbles.(21) The ultrasonic frequencies used in the acoustic flow cytometer have a wavelength $(4 \mu \mathrm{m})$ in the range of the size of the microbubbles, which makes the flow cytometer system a suitable candidate for the analysis. In addition to analysis of microbubble diameters, there is a gap in the characterization of targeted microbubbles, which are used as drug carriers to a specific area of a body for therapeutic applications. I believe that the photoacoustic signals at ultra-high frequency can be used to study the attached molecules and also the overall targeted microbubbles as these molecules have been shown to absorb a specific wavelength of light.

The research presented in this thesis demonstrates the development of a system that rapidly detects and analyzes the signals that help us to understand the interaction of ultra-high frequency and individual cells or microbubbles, and to develop a microfluidic technique to generate microbubbles of desired sizes for ultrasonic applications. The signals generated can be used for label-free clinical diagnostic applications, such as detecting circulating tumor cells in a bloodstream or to detect foreign cells in various bodily fluids. Currently, there is no commercial method that rapidly screens the label-free samples to detect 
these rare cells. In addition to diagnostic applications, this system can be used in basic scientific research on single biological cells. I believe that extending the application of the acoustic flow cytometer to size and characterize microbubbles the developed system's reach and impact can be increased.

\subsection{Background}

In this section, some basic theoretical concepts of the tools and techniques used in this research are introduced. These theoretical concepts will provide a basic understanding of the acoustic cytometry system that is presented in this thesis.

\subsubsection{Microfluidics}

Microfluidics is the study of the behavior, manipulation, and control of fluids that are confined to microstructures. Fluid volumes in this confinement range from microliter to picoliter. The fluid in such micro-domains show unique physical phenomena, compared to that in the macro-domain. These phenomena include: presence of laminar flow, the dominance of capillary forces, and diffusion limited natural mixing. Manipulation of the confined fluid is possible using various microfluidic platforms, such as pressure driven microfluidics, centrifugal microfluidics, lateral flow microfluidics, and droplet microfluidics.(22) One of the most common microfluidic platforms that is used in this research is the pressure-driven microfluidic platform. In the pressure-driven platforms, a positive pressure drives the flow in the microchannels, which obey the no-slip boundary condition (the velocity of the fluid located at the channel walls is zero). Hence, the flow profile is parabolic with velocity reaching its maximum at the center of the channel. (22)

\section{Laminar flow}

There are two fundamental types of fluid flows: laminar and turbulent. Laminar flow behaves like a stack of fluid layers sliding over each other without any disturbances. In contrast, in turbulent flows, the fluid circulates due to the inertial effect of the fluid. Mixing in the laminar flow regime is limited by diffusion, whereas more active mixing is supported by turbulence in the turbulent flow regime.(23) Pressure-driven flows in microfluidic devices are laminar flows, in general.

The motion of fluid in a microchannel is governed by two main equations, namely the continuity equation and the Navier-Stokes equation. The continuity equation is a general form of the conservation of mass, which states that the mass entering through a controlled volume is equal to the mass leaving the volume for incompressible and steady flow. Thus, the density of the fluid, $\rho$, remains constant in time and space. The continuity equation for the continuous microfluidic flow of Newtonian fluids is:

$$
\frac{\partial \rho}{\partial t}+\nabla \cdot(\rho \mathbf{u})=0
$$


where $\mathbf{u}$ is the flow velocity, $\rho$ is the density, $\mu$ is the viscosity of a moving fluid, and $\nabla$ is the del operator. Using the incompressibility condition, the continuity equation (Eq. 1.1) reduces to

$$
\boldsymbol{\nabla} \cdot \mathbf{u}=0
$$

Similarly, the equation describing the momentum conservation principle for fluid particles is called the Navier-Stokes equation, which states that the change in momentum in a control volume is equal to the sum of forces applied to the volume. The Navier-Stokes equation for an incompressible fluid flow is expressed as

$$
\rho\left(\frac{\partial \mathbf{u}}{\partial t}+(\mathbf{u} \cdot \nabla) \mathbf{u}\right)=-\nabla p+\mu \nabla^{2} \mathbf{u}+\boldsymbol{f}
$$

where $f$ represents the body forces applied to the fluid volume. The left-hand side of the equation contains inertial terms and the right-hand side is equal to the sum of the pressure gradient, viscous force, and other forces such as gravity and electrostatic force. The Navier-Stokes equation expressed in Eq. 1.3 can be reduced to different simplified forms depending upon the assumptions made for the flowing liquid. The details can be found in the literature.(22)

The Navier-Stokes equation is complex for arbitrary flows. To characterize the arbitrary flows, dimensionless numbers are used. These dimensionless numbers represent the behavior of the dynamic system which are independent of the units of the physical parameters used to define the system. Experiments with similar values of such dimensionless numbers are dynamically alike and behave similarly. One of the dimensionless numbers is the Reynolds number $(R e)$, which is derived by non-dimensionalizing the Navier-Stokes equation.(22) Quantitatively, the $R e$ is defined as the ratio of the inertial forces to the viscous forces as expressed in Eq. 1.4

$$
R e=\frac{\rho u l}{\mu},
$$

where $l$ is the characteristic length of an object in a flowing fluid. In typical microfluidic flow conditions, the $R e<<1$ due to the small-scale and low-velocity nature of microfluidic flows. Therefore, in most microfluidic conditions, the inertia of the flow is neglected. Hence, Eq. 1.3 simplifies to

$$
0=-\nabla p+\mu \nabla^{2} \mathbf{u} .
$$

\section{Hydrodynamic flow focusing}

The interrogation of individual cells or particles with ultrasound and photoacoustics is possible when the particles flow through the focal zone of the ultrasound transducer in a single stream. The hydrodynamic flow focusing is used to stream cells or particles in a single file. Furthermore, the hydrodynamic focusing is important to the acoustic flow cytometry application because the sample has to be in a liquid to allow for the surrounding liquid to be used as the coupling fluid between the sample and the 

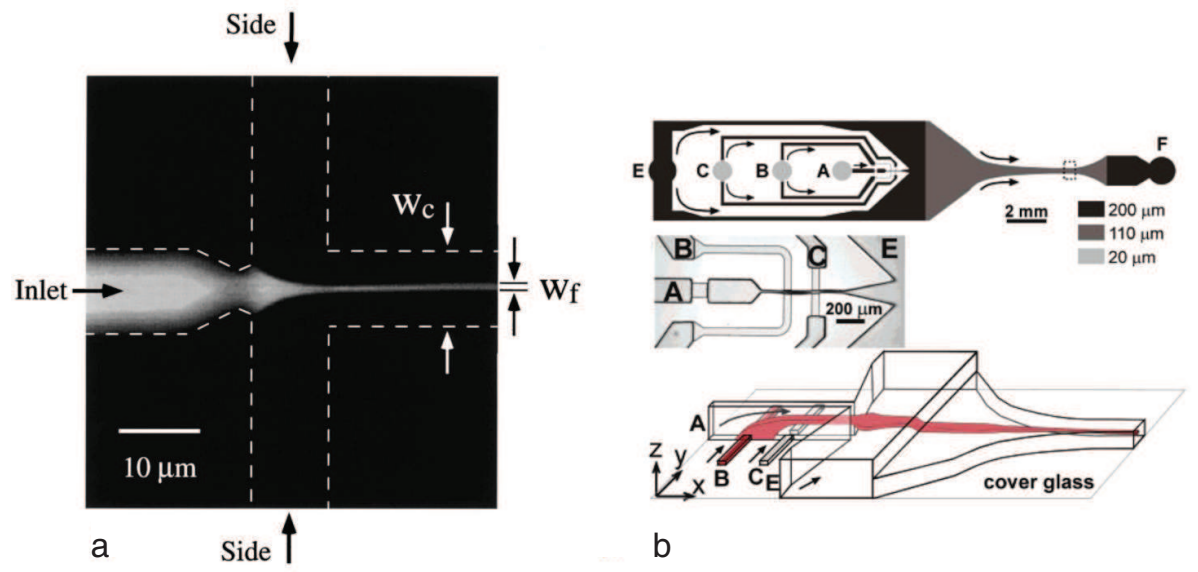

Figure 1.1: Hydrodynamic flow focusing in microfluidic devices. a) Schematic of a two-dimensional flow focusing design where flow from the inlet is focused in lateral directions by the sheath flows from the side channels. In the figure, $W_{f}$ is the width of focused flow and $W_{c}$ is the width of the focusing channel adopted from (25). b) A three-dimensional flow-focusing design. The top figure shows the top view of the design adopted from (26). Inlets $A, C$, and $E$ are for sheath flows and $B$ is for the sample flow and the height of the channels are color coded. The middle figure shows the segment of the design where the flows meet. The lower figure shows the design in three dimensions with red indicating the sample flow being focused downstream of the microchannel.

ultrasound transducer. Due to the negligible inertia in most microfluidic flows, ordering or focusing particles inside microfluidic channels require methods that do not rely on inertia. One example is hydrodynamic focusing, which is a process used to focus a sample fluid using sheath flows that are flowing faster than the sample flow. Such flow focusing in a microchannel is achieved by squeezing a sample flow from either the lateral direction (two-dimensional, 2D) or from both the lateral and vertical directions (three-dimensional, 3D).(24) In the flow focusing process, the sample flow is continuously squeezed by the sheath flows. Hydrodynamic flow focusing can be achieved by different techniques. $25,26,27,28$, 24) Fig. 1.1 shows examples of a $2 \mathrm{D}$ flow focusing $(25)$ and a $3 \mathrm{D}$ flow focusing $(26)$ taken from the literature. In Fig. 1.1 a, a sample flow is hydrodynamically focused in lateral directions using two sheath flows from two side inlets. The sample flow from the center inlet is squeezed from both sides resulting in a reduced width. Similarly, Fig. 1.1p shows a second hydrodynamic technique in which the sample flow is focused in both the lateral and vertical directions. To achieve 3D flow focusing, four sheath flows were introduced. Two sheath flows (labeled $\mathrm{A}$ and $\mathrm{C}$ in the figure) flow above and below the sample flow (labeled B), focusing it vertically. The flow from an inlet (labeled E) divides into two sheath flows to focus the sample flow to approximately $5-10 \mu \mathrm{m}$ in the lateral direction of the flow.

\subsubsection{Ultrasound and photoacoustics}

In an acoustic flow cytometer, the sample particles that are flow focused interact with ultrasound and laser pulses. These interactions generate ultrasound backscatter and photoacoustic waves. The following 
paragraphs highlight the basic principles of ultrasound and photoacoustics.

\section{Ultrasound}

Ultrasound waves are mechanical waves described by a change in pressure and displacement of particles in a medium. The frequency of ultrasound waves is above the human audible range of $20 \mathrm{~Hz}$ $20 \mathrm{KHz}$. Ultrasound waves have been used for biomedical imaging since Dr. Karl Dussik developed the imaging technique in 1942.(29)

When an ultrasound wave interacts with a sample, it can be reflected, scattered, refracted, and attenuated. The reflection of the ultrasound wave depends on the acoustic impedance mismatch between the coupling medium and the sample, size of the sample, and the angle of incidence of the wave. When the size of a sample is less than a wavelength of the ultrasound, the incident wave is scattered. The scattered wave that returns back to the transducer (i.e. scattering angle of $180^{\circ}$ ) is called ultrasound backscatter. The backscattered signal and the known value of the speed of sound in the sample (eg. cell/tissue) are used to generate ultrasonic images. Ultrasound imaging is relatively fast, radiation-free, and inexpensive compared to the other imaging techniques such as MRI and X-Rays. 30, 31, 32)

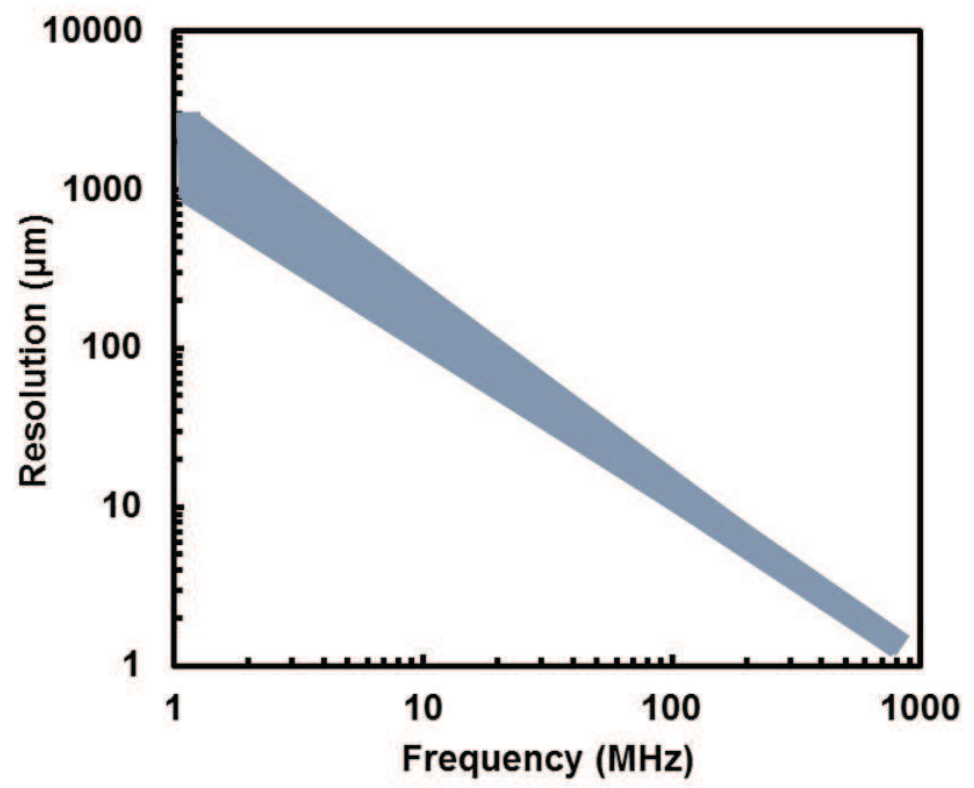

Figure 1.2: Ultrasound imaging resolution as a function of the ultrasound wave frequency. (Courtesy of Dr. Eric Strohm)

Ultrasound resolution highly depends on its frequency. Higher frequencies result in better resolution. The relation between the frequency and the lateral resolution is shown in the graph in Fig. 1.2. The resolution reaches few micrometers when the frequency is above $100 \mathrm{MHz}$ for single element transducers. 
Commercial medical ultrasound instruments use frequencies up to $15 \mathrm{MHz}$, however, some preclinical instruments have frequencies measuring up to $50 \mathrm{MHz} .(33,34)$ The resolution of clinical ultrasound devices is around $100 \mu \mathrm{m}$ when using frequencies of $15 \mathrm{MHz}$. To achieve resolution as low as a few micrometers, such as when detecting a typical single cell, the frequency needs to be above $100 \mathrm{MHz} .(35)$ However, the penetration depth of the system decreases from few millimeters to micrometers (when frequency changes from $100 \mathrm{MHz}$ to $1 \mathrm{GHz}$ ), which limits its application for many clinical uses.(36)

The acoustic microscope developed by Lemons at el. has sub-micrometer resolution and is able to probe single cells.(37) Thereafter, extensive research on single, fixed, and live cells has been published using acoustic microscopy.(35) To achieve resolution at the cellular level, acoustic microscopes use high frequencies above $100 \mathrm{MHz}$. The ultrasound waves used in the acoustic flow cytometer have a center frequency of $375 \mathrm{MHz}$, which has an ultrasound wavelength of approximately $4 \mu \mathrm{m}$. At this scale, the achieved frequencies are enough to detect and analyze individual cells and microparticles flowing through a microfluidic device.

\section{Photoacoustics}

Generation of pressure waves from an interaction of a particle with the electromagnetic radiation is known as the photoacoustic effect. In the context of the research presented in this thesis, photoacoustics is the principle of generating acoustic waves in response to an interaction of a particle or a biological cell with a short-pulsed laser.(38) These particles/cells absorb optical energy, which causes local heating within the absorber and a subsequent increase in temperature. The rise in temperature causes an increase in internal pressure leading to the thermoelastic expansion of the particle.(39) This expansion causes mechanical effects on the surrounding medium, which generates mechanical waves, called photoacoustic waves. Such photoacoustic waves can be detected by an ultrasonic transducer. The magnitude and frequency of these photoacoustic waves depend on the size, shape, and the density of the absorber, the speed of sound in the absorber, and the energy of irradiation. $40 ; 41 ; 42,43,44$

To generate efficient photoacoustic waves, two conditions must be satisfied: thermal confinement and stress confinement.(10, 45) To satisfy the thermal confinement condition, the laser pulses should be very short with a time duration that is smaller than the time required for thermal relaxation of the sample. Similarly, to satisfy the stress confinement condition, the thermal stress, produced by the optical absorption, should be limited to a region of thermoelastic expansion, and the relaxation time of the absorber should be longer than the laser pulse duration. These thermal and stress confinement conditions can be expressed in the following equations

$$
t_{\text {stress }}<\frac{d}{c}, t_{\text {thermal }}<\frac{d^{2}}{4 \alpha}
$$

where $d$ is the characteristic length scale of the object, $c$ is the speed of sound in the absorber and $\alpha$ is the thermal diffusivity.(46) Using these photoacoustic waves, it is possible to probe individual particles of micron and submicron size depending on the laser source and the properties of the transducer.(10) 
The detailed description of photoacoustic waves and modes of generation are well described in the literature. $8,40,47,48)$

a) Ultrasound

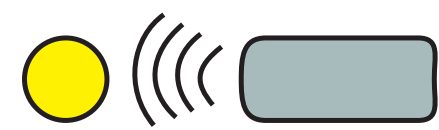

Particle Transducer

b) Photoacoustics
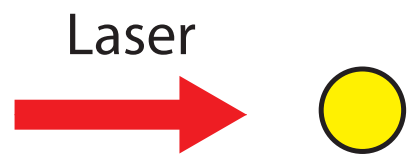
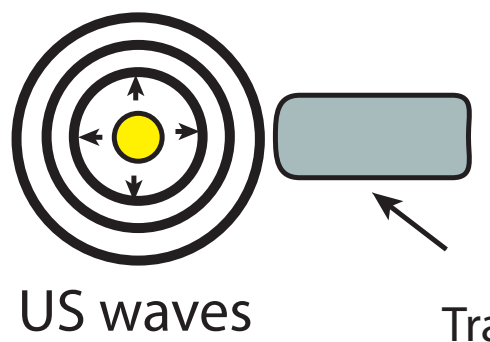

Transducer (detector)

\section{PA waves}

Figure 1.3: Schematic illustration of the generation of ultrasound backscatter and photoacoustic waves from a spherical particle. a) Ultrasound: the particle scatters the incident pressure waves, which are detected by the probing transducer as backscatter. b) Photoacoustics: a particle irradiated by a laser pulse absorbs the optical energy and undergoes thermal expansion to generate photoacoustic waves. The ultrasound transducer can also be used to detect the photoacoustic waves.

Fig. 1.3 shows a schematic illustration of the generation of ultrasound backscatter (Fig. 1.3 $\mathrm{a}$ ) and the photoacoustic waves (Fig. 1.3 b) from a particle. The interaction between the ultrasound and the particle, as well as the laser pulses and the particle, result in ultrasound backscattering and the generation of photoacoustic waves, respectively. An ultrasound transducer can be used to detect both signals. The detected signals can be post-processed to calculate the particle size, its mechanical properties, and its optical absorption properties. The capacity to characterize small (micron size) particles depends on the ultrasound frequency and the spot size of the laser.

The research presented in this thesis uses ultra-high frequency (bandwidth from $200-450 \mathrm{MHz}$ ) ultrasound and photoacoustic waves to detect and analyze signals from single cells with sizes in the micrometer range. A nanosecond laser with a pulse energy in the range of $100 \mathrm{~nJ}$ is used to irradiate the individual cells which produce the photoacoustic waves. The ultrasound and laser power used in this work can be considered biocompatible as it does not harm or breakdown biological samples.(10) The highfrequency ultrasound backscatter and photoacoustic systems enable high-resolution experimentation, use non-ionizing radiation, and the power used in these system do not damage or alter samples under study. Similar systems are used for clinical applications. $10,49,50)$ 


\section{Signal analysis}
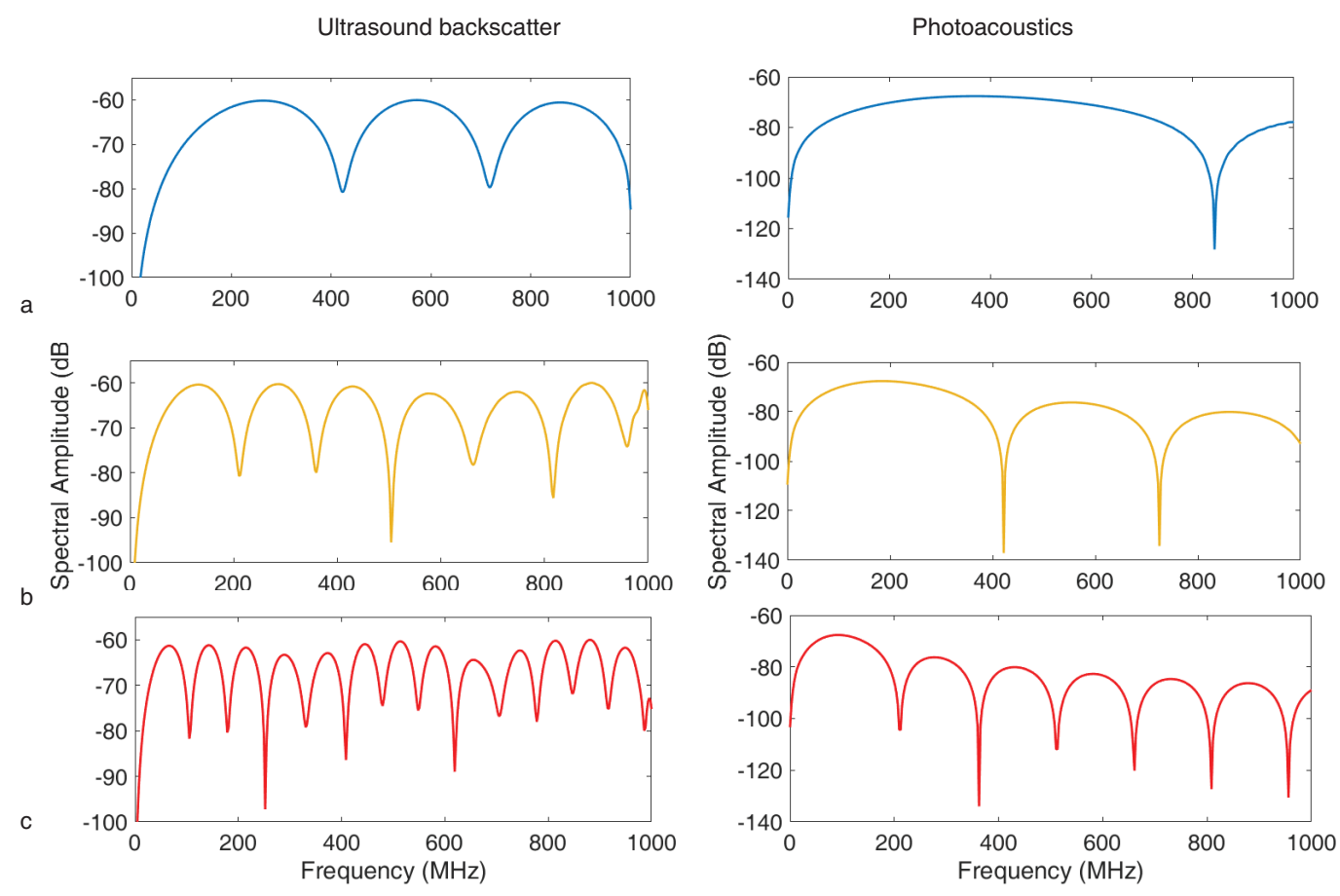

Figure 1.4: Frequency spectra of the ultrasound backscatter and photoacoustic signals from individual fluid spheres of varying diameters: a) $2.5 \mu \mathrm{m} \mathrm{b)} 5 \mu \mathrm{m}$ and c) $10 \mu \mathrm{m}$. The spacing between the minima is related to the size of the sphere.

The ultrasound backscatter and photoacoustic signals detected by an ultrasound transducer are typically subjected to a bandpass filter and a Hamming window to reduce noise content on the signals. The frequency spectrum of each processed signal is then calculated using the Fourier transform. The magnitude of the ultrasound backscatter and photoacoustic signals in the frequency domain from an individual scatterer show oscillatory patterns of minima and maxima, which are explained by V. Anderson and G. Diebold, respectively. (98, 40) Fig. 1.4 shows the frequency spectra of the ultrasound backscatter and the photoacoustic signals from individual fluid spheres of different diameters $(2.5 \mu \mathrm{m}, 5 \mu \mathrm{m}$, and $10 \mu \mathrm{m}$. The location (frequency) and the spacing between the frequency minima are related to the size of the scatterer/absorber for ultrasound backscatter and photoacoustics, respectively. The cells in the acoustic flow cytometry application are in suspension so that they can be approximated as a sphere. Hence, we can use these theoretical models to analyze the signals detected from the individual cells.

\subsubsection{Microbubbles}

Introducing contrast agents into the bloodstream increases the imaging contrast between the vessel and the surroundings. Microbubbles are one of the contrast agents used in clinical applications. 


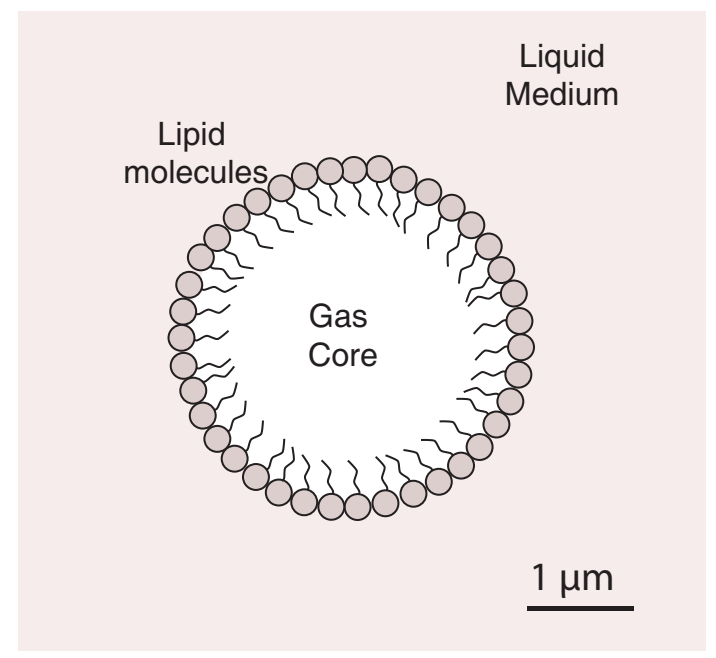

Figure 1.5: Schematic illustration of a microbubble with a gas core and lipid shell suspended in a liquid medium.

Microbubbles are gaseous objects, in which gas is typically encapsulated in a shell of either lipids, proteins, or polymers. $51,52,53$ ) Gases such as air, nitrogen, oxygen, and perfluorocarbon are used in the core. Perfluorocarbons are the preferred gas of choice due to its high ultrasound backscatter, strong hydrophobicity, and limited diffusion into the surrounding liquid. The structure of a typical microbubble is shown in Fig. 1.5. The diameter of these microbubbles ranges from 1-7 $\mu \mathrm{m}$. At resonance, these microbubbles become highly responsive to the ultrasound waves and result in strong backscatter.

In clinical applications, the microbubbles are intravenously injected into a patient. Selected ultrasound frequencies, in the order of a few $\mathrm{MHz}$, are used to resonate the microbubbles at the target region in the body. The resonance raises the scattering from the microbubbles and increases the contrast of the ultrasound images used for diagnostic imaging.(11) These microbubbles can be used for therapeutic applications, also, for which high-pressure ultrasound waves interact with microbubbles. The microbubbles absorb the acoustic energy and undergo cavitation, which can destroy the microbubble and create shock waves. $54,55,56,57)$ These shock waves enhance the membrane permeation of the cells and increase their molecular uptake.(55) Recently, microbubbles have been used as drug delivery agents to carry drug molecules and genes for targeted site-specific treatment. The bubbles are then destroyed by ultrasound at the site in vivo. This destruction increases cell membrane permeability and hence, increases the delivery efficacy of the drugs and/or genes.(58, 59) 


\subsection{Objective and hypothesis}

The main objective of this research is to develop an acoustic-based microfluidic flow cytometer that is able to detect and analyze individual micrometer-sized objects in a heterogeneous population using ultra-high frequency ultrasound backscatter and photoacoustics. Compared to the existing optical based flow cytometry systems, the acoustic-based system does not require staining of the cellular samples. The focus of the research is to develop a system capable of analyzing both biological cells as well as micrometer and sub-micrometer sized solid particles and gaseous bubbles.

The research hypothesis is: an acoustic-based microfluidic flow cytometer can be used to analyze label-free single cells in a heterogeneous population using simultaneous ultrasound backscatter and photoacoustic signals.

With the long-term objective of analyzing the individual cells and micro/nanobubbles, the proposed research has the following Specific Aims (SA):

1. SA1 Develop a microfluidic device that generates a very stable fluid flow, hydrodynamically focused in three dimensions, that allows cells to travel in a single file through the microchannel.

2. SA2 Develop an acoustic flow cytometer combining the device from SA1 with an ultrasound and photoacoustic system.

3. SA3 Develop a microfluidic technique to generate microbubbles ranging in sizes required for biomedical applications, i.e. from 1-7 $\mu \mathrm{m}$. Use these bubbles as samples for acoustic flow cytometer.

4. SA4 Use the acoustic flow cytometer developed in SA2 to differentiate between different particles and cells in a heterogeneous solution.

\subsection{Overview of the dissertation}

The research presented in this thesis focuses on the development of a label-free microfluidic flow cytometer to analyze single micrometer sized objects using simultaneous ultrasound backscatter and photoacoustic waves. To achieve this, we developed a stable flow focusing microfluidic system (Chapter 2 ), then coupled the microfluidic system with ultrasound and laser to characterize individual flowing cells using ultrasound backscatter and photoacoustics (chapter 3), and then applied the flow system to analyze microbubbles generated by the microfluidic system (Chapter 4). This is the first demonstration of a continuous flow technique that uses ultra-high frequency ultrasound backscatter and photoacoustics to analyze label-free single cells. As a result of continuous flow, the system's throughput is up to 150 cells/min.

The acoustic flow cytometer requires a stable and precisely controlled single stream of individual cells or particles. Chapter 2 describes the microfluidic technique that generates stable flow focusing of 
a sample of individual cells in a microfluidic channel. Here, we compare the stability of the focused flows generated using hydrostatic pressure with that of state-of-the-art syringe pumps. The results show that the stability of the focused flow achieved using hydrostatics is significantly better than the stability achieved using commercial syringe pumps. This finding is important for my research because the acoustic flow cytometer requires precise and controlled 3D flow focusing sample cells or particles.

Chapter 3 presents the label-free acoustic flow cytometer that was developed during my dissertation. Here, we demonstrate that the acoustic flow cytometer is capable of label-free analysis of individual particles and cells using simultaneously detected ultrasound backscatter and photoacoustic signals. The results of the label-free acoustic flow cytometer to differentiate blood cells show a strong agreement with the results from the same sample, tagged with fluorescent dye, using a commercial fluorescence activated cell sorting (FACS).

The application of the acoustic flow cytometer is not limited to single cell analysis. This system is capable of analyzing other submicron objects, such as micro or nanobubbles. In Chapter 4, I describe a novel technique for producing microbubbles in a microfluidic device and shrinking of those bubbles to a targeted size. This microbubble generation technique produces monodisperse microbubbles measuring hundreds of micrometers in diameter. Using a vacuum pressure, these bubbles can be shrunk to 1-7 $\mu \mathrm{m}$ in diameter. Both the generation and shrinkage of the bubbles are achieved in a single chip and in a single process.

Chapter 5 summarizes the results of the overall research and describes potential applications of the acoustic flow cytometer.

Appendix has a collection of additional information related to this research. Appendices A, B, and $\mathrm{C}$ are the supplementary information published with the papers included in Chapters 2, 3, and 4, respectively. Appendix D highlights ongoing research on two major applications: 1) calculating the nucleus to cytoplasmic (N:C) ratio of tumor cells using the acoustic flow cytometer to achieve the longterm goal of developing a label-free technique for detecting circulating tumor cells, and 2) analysis of microbubbles and nanobubbles using the acoustic flow cytometry. Appendix E lists the journal articles, conference proceedings, and conference presentations resulting from research performed during my $\mathrm{PhD}$ studies.

\subsection{Author's contribution in the context of collaboration}

The microfluidic acoustic flow cytometer presented in this thesis is designed and developed by the author. The author is also responsible for designing, prototyping, testing, and validating the microfluidic devices. The assembly, integration of ultrasound and laser systems and the electronics control system design is also done by the author. 
For the hydrostatic based stable microfluidic flow-focusing research presented in Chapter 2, the microfluidic device and the experimental setup was designed and fabricated by the author. The author outlined the experimental plan and performed most of the experiments. The data were analyzed by the author himself. An undergraduate research assistant, Mohammadali Saremi, helped to collect data for some of the experiments.

For the acoustic flow cytometer development presented in Chapter 3, the design, fabrication, and development of the microfluidic device were solely done by the author. The ultrasound and photoacoustic technique used in this research is based on the technique that was developed by the previous student and postdoctoral fellow, Dr. Eric Strohm. Integration of the modules including the microfluidic device, laser and optical system, ultrasound system, and the hardware control system was done by the author. The control software was updated from an existing version of SASAM software (used to control an acoustic microscope) with the help of Dr. Strohm. All the experiment planning, execution, data acquisition and data analysis were performed by the author. Undergraduate research assistants, Parminder Saggu, Mia van de Vondervoort, Radi Abubaker, Caryn Geady, Andrea Stobo, Robert Ngunjiri, Joseph Sebastian, and Oliver Wang, helped the author during the project period.

For the microbubble generation and shrinking research presented in Chapter 4, the idea was developed together with a previous postdoctoral researcher (Dr. Byeong-Ui Moon, co-first author of the paper). The original experiment was done by Dr. Moon, with the follow-up device development, experimental plan, experimental setup design, execution of the planned experiments, and data analysis done by the author. In this research, an undergraduate research assistant, Jennifer Kieda, helped the author to collect and analyze the data. 



\section{Chapter 2}

\section{Stable microfluidic flow focusing}

The work presented in this chapter is based on the following article published in a peer reviewed journal Biomicrofluidics.

Gnyawali, V., Saremi, M., Kolios, M. C., and Tsai, S. S. H. (2017). Stable microfluidic flow focusing using hydrostatics. Biomicrofluidics, 11(3), 34104. https://doi.org/10.1063/1.4983147

\section{Author's contribution}

The microfluidic device and the experimental setup was designed and fabricated by the author. The author outlined the experimental plan and performed most of the experiments. The data were analyzed by the author himself. An undergraduate research assistant, Mohammadali Saremi, helped to collect data for some of the experiments.

\section{$2.1 \quad$ Introduction}

The development of microfluidic techniques in recent years is leading to an emergence of enhanced lab-on-a-chip (LOC) devices. (60; 61) Such LOC devices demonstrate the potential of replacing existing larger scale technologies in bio-chemical, bio-physical, and bio-medical processes. 62,$63 ; 64)$ A major advantage of LOC devices is the ability to provide a quasi-natural environment to cells that are under observation by controlling flow properties such as laminarity, 65 , 22) physical parameters such as flowrate, $(66 ; 22)$ and the size of the microchannels. (22) The application of hydrodynamic focusing in these microfluidic flows provides an additional advantage by narrowing the flows toward the size of a single cell. (67) For example, in a flow cytometer, (68) single cells are hydrodynamically flow focused to a narrow stream that passes through an interrogating zone where the individual cells are interrogated by optical (69) or electrical systems (6, 70) for diagnostics and sorting applications. In addition to flow 
cytometry, focused flows are also useful in applications such as drug discovery, (71, 72) drug delivery and release,(71) deoxyribonucleic acid (DNA)-stretching, (73) and reagent mixing in microfluidics.(25)

Typical microfluidics experiments use constant flow-rate syringe pumps to drive the fluid flow.(64) The flows generated by syringe pumps visually appear to be precise, (74) but measurements show that the diameter of focused flows from syringe pumps can fluctuate significantly: one recent publication shows radial variation of $13 \mu \mathrm{m}$ when the average diameter is $217 \mu \mathrm{m}$.(75) The authors detect the fluctuations of syringe pumps by tracking the interface of ultralow interfacial tension aqueous two phase systems (ATPS) and demonstrate that the fluctuations are directly related to the steps of the stepper motor in the pumps. In a similar approach, another publication shows that the flow fluctuations are related to the pressure fluctuations in the syringe pumps.(76) These syringe pumps also produce periodic oscillations in the flows due to the frictional forces between the syringe piston and the syringe wall.(77)

These flow instabilities caused by syringe pumps may have a detrimental effect on the effectiveness of new and highly sensitive microfluidic flow cytometry methods.(78) Indeed, the requirements for narrow and consistent flow focusing in these newly developed microfluidic flow cytometry techniques are highlighted in a recent publication by Strohm et al., (79) where the researchers are attempting to characterize cells and particles using acoustics in microfluidics.

In this chapter, we demonstrate the application of hydrostatic pressure-driven flow to achieve precise and stable flow focusing. We compare experimental results from hydrostatics and syringe pump-driven flow focusing and find that hydrostatics achieves superior flow focusing stability in a range of focused widths. Finally, we show that our hydrostatics-based flow focusing technique is easily tunable to control the width of the focused flow. We anticipate that this stable and easily tunable hydrostatics-based flow focusing method will find utility in many sensitive microfluidic flow cytometry applications that require highly stable focused flows.

\subsection{Experimental methods}

\subsubsection{Device fabrication}

The microfluidic devices in our experiments are fabricated using the classical soft lithography technique. (80) A photomask is designed using computer-aided design (CAD) software (AutoCAD 2010, Autodesk, Inc., Dan Rafael, CA, USA), printed on a transparency sheet (25,400 dpi, CAD/ART Services

Inc., Bandon, OR, USA), and patterned on a silicon wafer in a single SU-8 layer using photolithography. The pattern is transferred to a polydimethylsiloxane (PDMS, Sylgard 184 silicone elastomer kit, Dow Corning, Midland, MI, USA) sheet. Inlet and outlet holes on the PDMS base are opened using a 1 mm diameter biopsy punch (Integra Miltex, Inc., Rietheim-Weilheim, Germany). The PDMS sheet is then bonded to a glass microscope slide by oxygen plasma treatment (Harrick Plasma, Ithaca, NY, USA). 


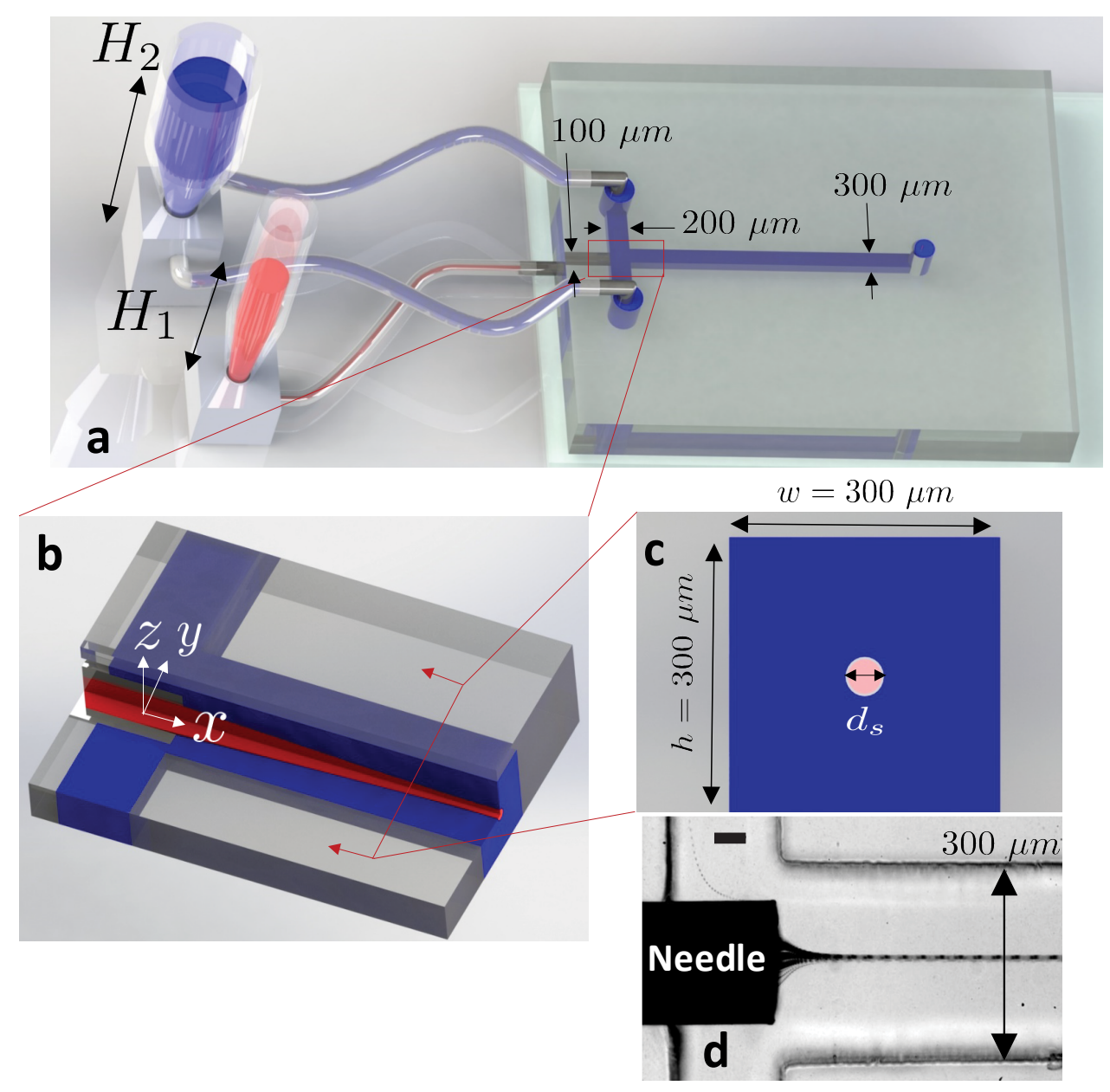

Figure 2.1: The hydrostatics-based microfluidic device. Schematic illustrations show that (a) fluid flow is driven by hydrostatic pressure that is controlled by the liquid column heights $H_{1}$ and $H_{2}$ of the sample (red) and sheath (blue) liquids, respectively. The sample flow is supplied to the microchannel via a needle that is inserted to the side of the PDMS slab and the sheath flow enters the microchannel via tubing connected from the top of the device. (b) Extended isometric section view of the flow focusing crossjunction shows that the sheath flow surrounds and flow focuses the sample flow. Sample and sheath fluids both flow in the direction indicated by the $x$-axis. (c) Cross section view of the microchannel downstream of the cross-junction shows the diameter, $d_{s}$, of the flow of the sample fluid. Experimental image showing (d) the flow focusing of the sample fluid near the cross junction. Scale bar represents 50 $\mu \mathrm{m}$. 
Upstream of the flow focusing cross-junction, the sheath flow channels and the sample channel are 100 and $200 \mu \mathrm{m}$ wide, respectively (Fig. 2.1 a). The channel downstream of the cross-junction is $w=300 \mu \mathrm{m}$ wide (Fig. 2.17). The height of all channels is $h=300 \mu \mathrm{m}$. To obtain flow focusing, a nano-needle (ID $=100 \mu \mathrm{m}, \mathrm{OD}=200 \mu \mathrm{m}$; Japan Bio Products, Tokyo, Japan) is manually guided through the sample inlet channel up to the cross junction (Fig. 2.1p). After inserting the needle, we align the needle in the lateral direction under a stereo microscope (E-Zoom 6V, Edmund Optics Inc., Barrington, NJ, USA). For vertical positioning, we place two layers of a thin transparent tape (3M, St. Paul, MN, USA) underneath the needle to achieve a $40 \mu \mathrm{m}$ gap between the needle and the bottom of the channel, resulting in a 60 $\mu \mathrm{m}$ gap above the needle. The sheath flows, above and below the sample flow, cause an axis-symmetric effect on the focused flow in both lateral $(x-y)$ and vertical $(x-z)$ axes (see the supplementary video at aip.scitation.org/doi/suppl/10.1063/1.4983147/suppl_file/esi_stable_flow_focusing_confocal.avi). Finally, the needle is bonded to the glass substrate and the guiding channel is sealed using two-component epoxy glue (Henkel Canada Corporation, Mississauga, ON, Canada). To connect the needle inlet to the liquid columns, we bind the outer end of the needle to Tygon tubing using the two-component epoxy glue.

\subsubsection{Experimental setup}

To drive the flow inside the microchannels, we introduce liquid columns containing the sample and sheath fluids. The hydrostatic pressure due to the liquid columns drives the sample and sheath flows. Measuring pipettes (Corning Inc., Corning, NY, USA ) with internal diameters $3 \mathrm{~mm}$ and $8 \mathrm{~mm}$ are used as sample and sheath liquid columns, respectively. The tapered end of the measuring pipettes are inserted into pre-fabricated PDMS cubes, and Tygon tubing is used to connect the cubes to the inlets of the microfluidic devices (Fig. 2.1 ). The sample and sheath flow liquid columns have heights $H_{1}$ and $H_{2}$, respectively. To compare the results from hydrostatics-based flow focusing with classical syringe pump based flow focusing, we also perform experiments using the same microfluidic geometries with Pump 11 Elite syringe pumps (step resolution $=0.9^{\circ}$; Harvard Apparatus, Holliston, MA, USA).

We use permanent black ink (Higgins, Chartpak Inc., Leeds, MA, USA) as sample fluid and deionized (DI) water as sheath fluid for all of our experiments. The ink is filtered through a syringe filter (Corning Inc., Corning, NY, USA), with $0.45 \mu \mathrm{m}$ diameter pores. The color contrast between the ink and the DI water provides clear visualization of the focused sample flow. Fig. 2.1 $\mathrm{d}$ is a representative experimental image of the flow focusing of a narrow sample fluid in our microfluidic system. Here, we include $3 \mu \mathrm{m}$ diameter polystyrene particles in the sample fluid to further enhance the imaging contrast.

The focused flow is imaged using a high-speed camera (Phantom M110, Vision Research, Wayne, NJ, USA) attached to an inverted microscope (Olympus Corporation, Tokyo, Japan). For all of the experiments, the objective is focused at a region approximately $15 \mathrm{~mm}$ downstream of the cross-junction. We measure the focused flow diameter, $d_{s}$, at the same location in the microchannel for all of our experiments. We use an in-house Matlab program to extract individual frames from the videos and 
analyze the intensity of each pixel in each frame. The program uses the intensity values to measure the focused flow diameter, $d_{s}$, for each individual frame and calculate the average focused flow diameter. We use the Otsu greyscale thresholding algorithm for intensity thresholding in the frames. (81)

\subsection{Result and discussion}

\subsubsection{The stability of hydrostatics and syringe pump based flow focusing}

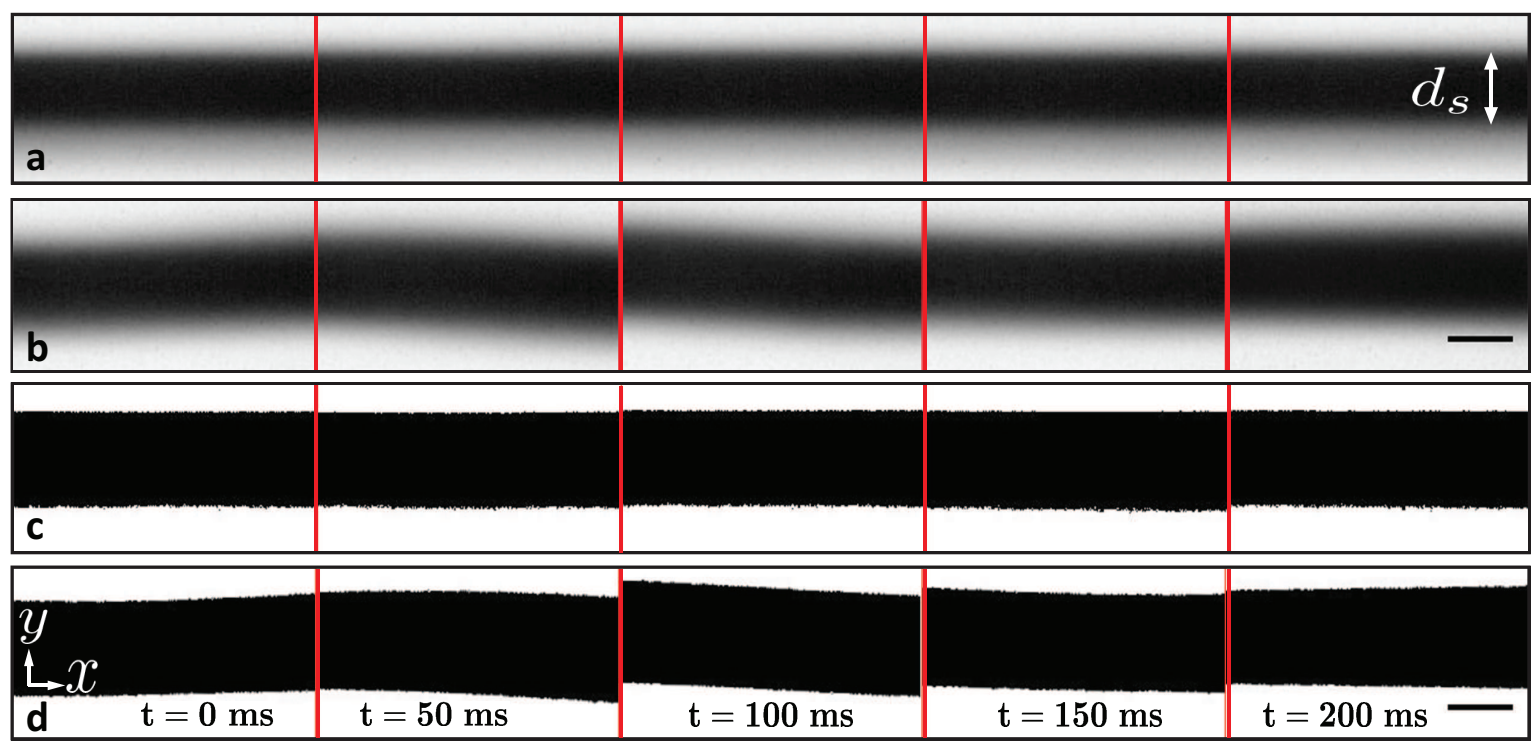

Figure 2.2: Experimental images of the sample fluid (black) focused inside a microchannel downstream of the focusing junction. These images are taken from video frames of the experiments whose focused flow average diameter, $d_{s}=59 \mu \mathrm{m}$, and the frames are selected at an interval of $50 \mathrm{~ms}$. Brightfield images of the focused flows controlled by (a) hydrostatic pressure and (b) constant flow-rate syringe pumps. Thresholded images of the same focused flows produced with (c) hydrostatic pressure and (d) constant flow-rate syringe pumps. The image sequences show qualitatively that the hydrostaticscontrolled focused flow has a more consistent diameter, $d_{s}$, than syringe pump driven flow. Syringe-pump driven focused flows also exhibit more erratic flow behavior. Flow is in the direction indicated by the $x$-axis. Scale bars represent $50 \mu \mathrm{m}$.

In our comparison of hydrostatics and syringe pump based microfluidic flow focusing, we use a baseline sample flow diameter, $d_{s}=59 \mu \mathrm{m}$, to compare the stability of both systems under equally narrow flow focusing requirements. To attain the focused flow diameter, $d_{s}=59 \mu \mathrm{m}$, we apply sample and sheath liquid column heights, $H_{1}=180 \mathrm{~mm}$ and $H_{2}=55 \mathrm{~mm}$, respectively, in the hydrostatics experiment, and sample and sheath flow rates, $Q_{1}=1.5 \mu \mathrm{L} / \mathrm{min}$ and $Q_{2}=30 \mu \mathrm{L} / \mathrm{min}$, respectively, in the syringe pump experiment. 
Fig. 2.2 shows representative sequential images from the same section of the focused sample flow taken from experimental videos. These sequential images are extracted at an interval of $50 \mathrm{~ms}$ apart. The dark sections indicate the sample flow, and the bright sections represent the sheath fluid. Figs. $2.2 \mathrm{a}$ and $2.2 \mathrm{~b}$ are bright field images and Figs. $2.2 \mathrm{k}$ and $2.2 \mathrm{~d}$ are the binary version of the same images after the Otsu thresholding.

The experimental thresholded images from hydrostatics based focusing (Fig. 2.2 c) shows a consistent focused diameter $d_{s}$, both spatially and temporally, in comparison with the thresholded images from syringe pump experiments (Fig. $2.2 \mathrm{~d}$ ). Additionally, the syringe pump based focused flow exhibits more erratic behavior. For example, the frame taken at $100 \mathrm{~ms}$ shows the focused flow tilting at an angle relative to the longitudinal axis of the flow. As hypothesized in previous reports on microfluidic flows with syringe pumps, the variation of the focused width in the syringe pump experiments may arise from the pulsation of the stepping mechanism in the pumps, 75,76 ) and the tilted focused flow may be due to stiction between the syringe piston and the syringe walls.(77)

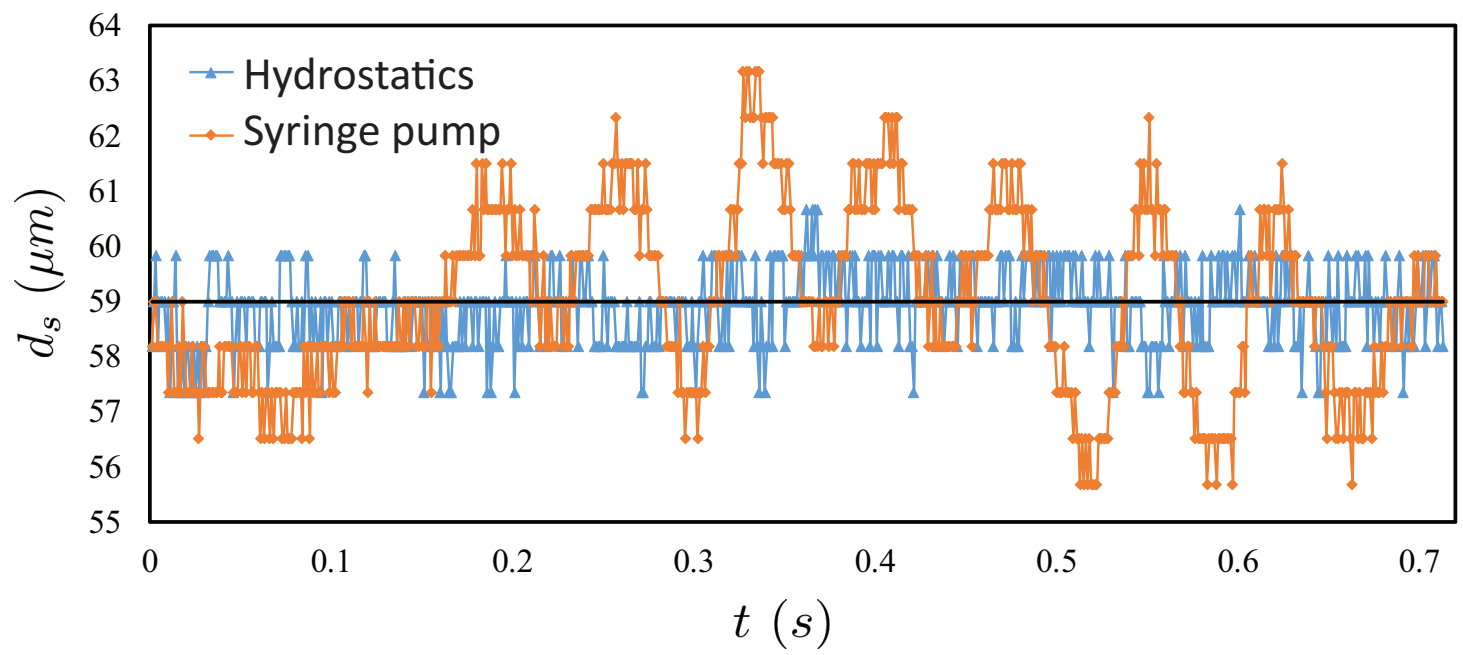

Figure 2.3: A plot of the focused flow diameter, $d_{s}$, versus time, $t$, for experiments using hydrostatic pressure (blue triangle) and syringe pumps (red diamond) over a period $\Delta t=0.7 \mathrm{~s}$. The average diameter, $d_{s}$, of the focused flow in both hydrostatics and syringe-pump experiments is $d_{s}=59 \mu \mathrm{m}$ (indicated by the black line). $t=0 \mathrm{~s}$ represents the first frame of the high speed video. The data shows significantly larger fluctuations in the syringe pump experiments.

For a quantitative comparison of the stability of the focused flows from the two experimental setups, we plot the focused sample flow diameter, $d_{s}$, versus time, $t$ (Fig. 2.3). Here, the average focused flow diameter $d_{s}=59 \mu \mathrm{m}$. Blue triangles show experimental data from hydrostatics experiments, and red diamonds show data from syringe pump based experiments. Fig. 2.3 demonstrates that hydrostatics 
based flow focusing results in consistently lower variation of the focused flow diameter, $d_{s}$, when compared to syringe pump based experiments. The standard deviation of the focused flow diameter, $d_{s}$, is $0.7 \mu \mathrm{m}$ for hydrostatics, versus $1.6 \mu \mathrm{m}$ for the syringe pump experiment.

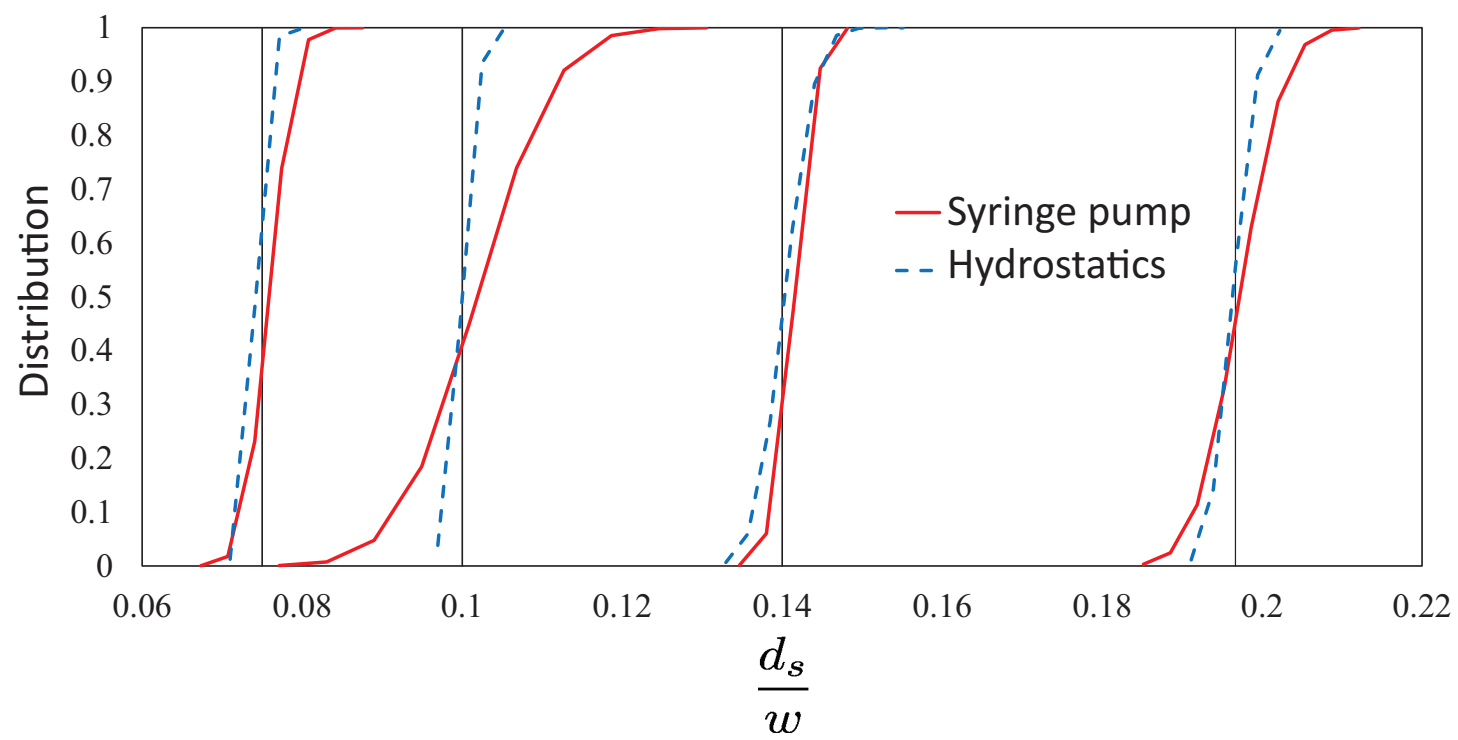

Figure 2.4: A plot of the cumulative normal distribution function versus dimensionless focused flow diameter, $d_{s} / w$, in the microfluidic channel driven by hydrostatic pressure (blue dashed lines) and syringe pumps (red solid lines). The four sets of data correspond to averaged focused flow widths, $d_{s} / w$ $=0.075,0.100,0.140$ and 0.197 (from left to right). Hydrostatics-controlled flows show consistently narrower distributions of the focused width, $d_{s} / w$, compared to the focused flow generated by syringe pumps.

Fig. 2.4 shows a plot of the cumulative distribution function of the dimensionless focused flow diameter, $d_{s} / w$, for cases where $d_{s} / w=0.075,0.100,0.140$ and 0.197 . Here, blue dashed lines show data from hydrostatics experiments and red solid lines show data from syringe pump experiments. Black solid vertical lines indicate the mean values of $d_{s} / w$. The distribution of the focused flow diameter is measured from individual diameters of the focused flow in each frame of the videos taken from the experiments. For hydrostatics experiments, we record videos for a duration of $0.7 \mathrm{~s}$ at a frame rate of 1,000 fps, and for the syringe pump experiments the videos are recorded for a duration of $16 \mathrm{~s}$ at frame rate of $200 \mathrm{fps}$.

The curves in Fig. 2.4 show that the cumulative normal distributions of the focused flow diameter in the hydrostatics experiments are consistently narrower for all of the experiments. The distributions of the focused flow diameter in the pump experiments are inconsistent and do not show any specific trend in the narrowness of the flow. For example, the experiments with $d_{s} / w=0.140$ show that the distributions of the syringe pump and hydrostatics experiments are nearly the same, while for $d_{s} / w=$ 0.100 the pump experiment results in a significantly wider distribution than the hydrostatics experiment. 
These results suggest that the hydrostatic flow focusing technique is better than focusing a flow using syringe pumps for sensitive applications that require accurate and consistent focused flows. We also find that the hydrostatics-based system maintains a near constant focused flow diameter, $d_{s}$, for at least several minutes into each experiment, before increasing due to changes to the liquid column heights of the sample and sheath fluids with time (see details in Appendix A). We note that these changes could be reduced by continually filling the liquid columns with liquid, or by increasing the diameter of the liquid columns. The liquid columns can be continuously supplied with liquid using a liquid reservoir connected to the columns. Alternatively, a continuous liquid supply can be achieved by using a syringe pump to infuse the liquid at a flowrate equal to the rate at which the column is discharging liquid to the microfluidic device. These methods may help to maintain constant fluid column heights.

\subsubsection{Controlling hydrostatics based flow focusing}

In syringe-pumped based flow focusing systems, the focused sample flow diameter scales as, $(82)$

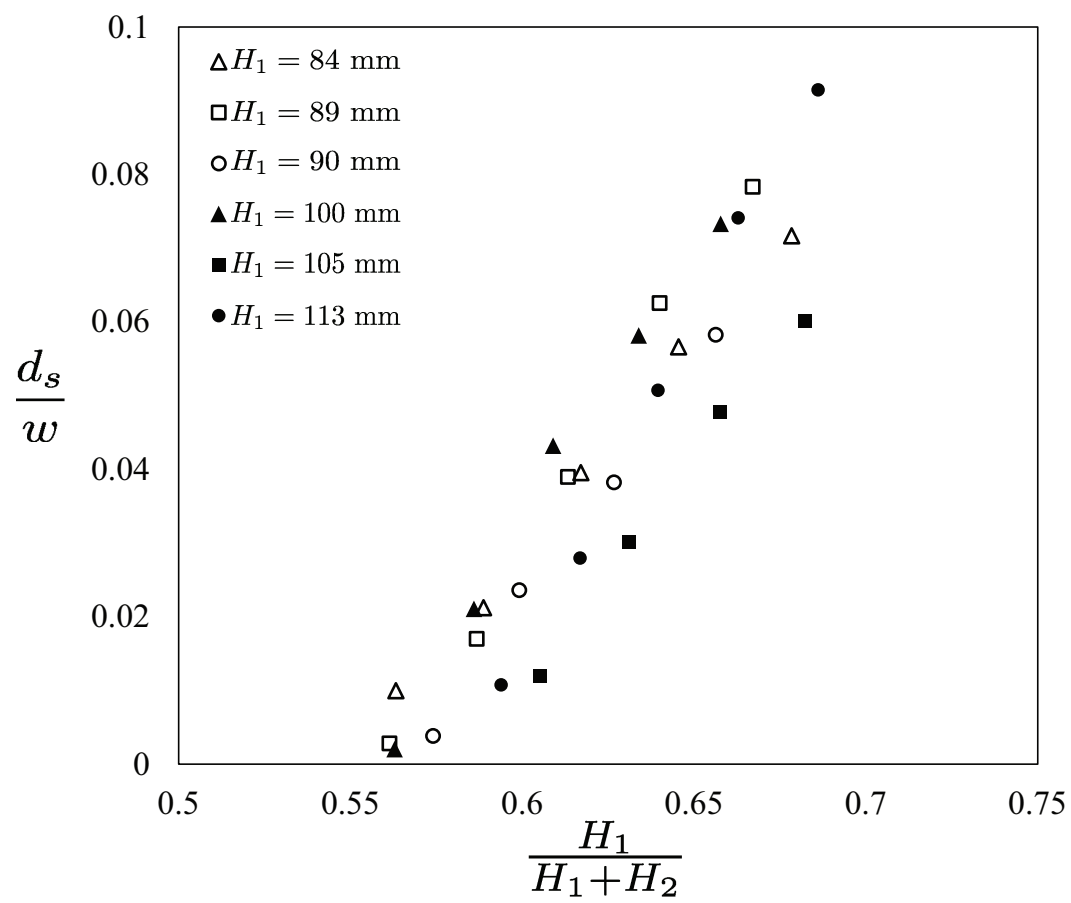

Figure 2.5: A plot of the dimensionless focused sample flow diameter, $d_{s} / w$, versus the ratio of liquid column heights, $\frac{H_{1}}{H_{1}+H_{2}}$. Here, the data shows results from six experiments where the sample liquid column height, $H_{1}=84,89,90,100,105$, and $113 \mathrm{~mm}$, and the sheath flow liquid column height, $H_{2}$, is varied to achieve different focused sample flow diameters, $d_{s} / w$. The results indicate monotonic and approximately proportional increase of the focused sample flow diameter, $d_{s} / w$, with the ratio of liquid column heights, $\frac{H_{1}}{H_{1}+H_{2}}$, suggesting that the parameter $\frac{H_{1}}{H_{1}+H_{2}}$ is a good controller for the degree of hydrostatics based flow focusing. 


$$
\frac{d_{s}}{w} \propto \frac{Q_{1}}{Q_{1}+Q_{2}},
$$

where $Q_{1}$ and $Q_{2}$ are the sample and sheath fluid flow rates, respectively. The Poiseuille flow relationship has a linear proportionality between the flow rate, $Q$, and the pressure drop, $\Delta P$, such that $Q \propto \Delta P$. Hydrostatics dictates that the pressure drop, $\Delta P$, scales linearly with the height, $H$, of a liquid column. Therefore, our hydrostatically flow focused sample fluid will have a resulting normalized diameter,

$$
\frac{d_{s}}{w} \propto \frac{H_{1}}{H_{1}+H_{2}} .
$$

Fig. 2.5 shows the dimensionless focused sample flow diameter $d_{s} / w$, plotted against the ratio of liquid column heights, $\frac{H_{1}}{H_{1}+H_{2}}$. Here, in six experiments, we fix the sample fluid's liquid column height $H_{1}=84,89,90,100,105$, and $113 \mathrm{~mm}$, and tune the sheath flow column height, $H_{2}$, to obtain a range of dimensionless focused sample flow diameters $d_{s} / w$. In all of the experiments, we observe that the sample flow diameter, $d_{s}$, is approximately proportional to the parameter $\frac{H_{1}}{H_{1}+H_{2}}$. Therefore, these results suggest that the sample flow diameter, $d_{s}$, is directly controllable by simply adjusting the ratio $\frac{H_{1}}{H_{1}+H_{2}}$.

\subsection{Conclusion}

In this work, we describe a stable hydrostatic pressure actuated hydrodynamically focused flow in a single layer microfluidic device. We validate the stability of the focused flow by comparing our results with values from conventional syringe pump actuated flow focusing. Overall, our results show that hydrostatics actuated flow focusing is more stable than syringe pump based flow focusing.

Additionally, we find that hydrostatic based focused flow diameter, $d_{s}$, is easily adjustable by tuning the ratio of the heights of the liquid columns, $\frac{H_{1}}{H_{1}+H_{2}}$, which enables simple control by changing a single parameter. We expect that the stability and ease-of-control of this flow focusing technique will make it highly useable in a variety of microfluidic flow cytometer systems.

\section{Supplementary material}

See supplementary material (Appendix A) for more information on hydrostatic experiment on controlled focus flow diameter and for a confocal microscopy video (aip.scitation.org/doi/suppl/10.1063/ 1.4983147/suppl_file/esi_stable_flow_focusing_confocal.avi) showing an axis-symmetric effect on the focused flow in both lateral $(x-y)$ and vertical $(x-z)$ axes. 



\title{
Chapter 3
}

\section{Acoustic flow cytometer development}

The work presented in this chapter is based on the following article submitted to a peer reviewed journal Scientific Reports.

Gnyawali, V., Strohm, E. M., Wang, J., Tsai, S. S. H., Kolios M. C. (2018) Microfluidic acoustic flow cytometry: a label-free approach, Scientific Reports. (submitted)

\begin{abstract}
Author's contribution
Design, fabrication, and development of the microfluidic device were solely done by the author. The ultrasound and photoacoustic technique used in this research is based on the technique that was developed by the previous student and postdoctoral fellow, Dr. Eric Strohm. Integration of the modules including the microfluidic device, laser and optical system, ultrasound system, and the hardware control system was done by the author. The control software was updated from an existing version of SASAM software (used to control an acoustic microscope) with the help of Dr. Strohm. All the experiment planning, execution, data acquisition and data analysis were performed by the author. Undergraduate research assistants, Parminder Saggu, Mia van de Vondervoort, Radi Abubaker, Caryn Geady, Andrea Stobo, Robert Ngunjiri, Joseph Sebastian, and Oliver Wang, helped the author during the project period.
\end{abstract}

\subsection{Introduction}

For many decades, flow cytometry has been one of the most effective and powerful approaches for rapidly analyzing the characteristics of single-cells in a large population. $69,83,84$ ) Flow cytometers use either optical or electrical impedance based techniques to analyze cell populations. $(70,85,86)$ Side and forward scattering of light from suspended individual cells, and fluorescence emission from intracellular 
components tagged with fluorescent molecules are the signal sources in optical-based techniques. (87) When the cells flow through an interaction zone, they interact with a laser beam, which causes the generation of the signals.(88) Forward scattered light is associated with cell size, side scattered light correlates with the structural complexity of the cells, and fluorescence signals relate to the type of intracellular components in the cells.(69) In impedance-based flow cytometers, the electrical impedance changes when a cell passes through an electric field. This change is correlated with the volume of the cell. (4, 5, 89)

Despite being ubiquitous in biomedical research, these classical flow cytometry approaches have several limitations. Light scattering from cells in optical-based cytometers provide information limited to cell size and granularity. (88) To detect DNA or RNA content, proteins, or antigens other molecules, such as a fluorescent dye or fluorescence-tagged antibodies, are attached to either specific intracellular components or to the cell membrane. The fluorescent signal can then be analyzed to extract the information of the cellular state by the detection of molecules or intracellular components to which the fluorescent molecules are bonded.(90) Some fluorescent markers can be cytotoxic.91) Moreover, the quality of the data generated when using fluorescent probes critically depends on sample preparation.(7) For some applications, such as gene expression and protein localization, higher fluorescence expression is required for higher optical resolution and sensitivity.(92, 93) On the other hand, impedance-based systems are only able to count and size cells and do not provide information of cell constituents.(9) Due to these limitations, there is ongoing research in developing new flow cytometry systems.

Ultrasound scattering can be used to analyze cells. $94,95,96,97)$ Acoustic waves above a frequency of $100 \mathrm{MHz}$ are highly sensitive to the cell's physical properties, such as shape and size, and biomechanical properties, such as Young's modulus. $(47 ; 98)$ Ultrasound waves generated as a response to optical irradiation are called photoacoustic waves. Photoacoustic waves are sensitive to the optical absorption properties of the cell and its constituents. $(45,79)$ The ultrasound and laser pulses can interact with a particle or cell to generate two distinct mechanical waves: ultrasound backscatter and photoacoustic waves. (47, 79, 98, 99) The former results from the acoustic impedance mismatch between the particle and the surrounding medium. 34,$35 ;, 100$ ) The latter results from the optical absorption of the particle, 45 ; 101) which generates a rapid thermoelastic expansion that creates broadband mechanical waves with frequencies in the ultra-high frequency (UHF) range. The generation and characterization of ultrasound backscatter and photoacoustic waves from solid and liquid spheres are well-described.(40; 98) This theoretical framework can be used in experimental measurements to infer properties of nearly spherical objects, for example, cells.

An advantage of combining ultrasound backscatter and photoacoustics in single cell analysis is the enabling of label-free and multi-parametric analyses based on two different physical interactions. (102) On the one hand, unique ultrasound backscatter signatures from individual cells provide information on cell size, shape, structure, and biomechanical properties. (36; 103) Existing reports of single cell analysis using acoustic microscopy demonstrate the capability to extract cellular properties. $(35,102 ; 104)$ On the 
other hand, photoacoustic signals contain information about the intracellular components that absorb the optical irradiation energy such as lipids, mitochondria, and deoxyribonucleic acid (DNA).(38; 105$)$ The source of the photoacoustic signal depends on the laser wavelength used and presence of the optically absorbing structures at those wavelengths. Since an ultrasound transducer is used to detect both ultrasound and photoacoustic waves, both ultrasound and photoacoustic can be used to probe the same cell.(106) However, in static microscopy systems the throughput is very low; typically on the order of a cell per minute.(96, 97) There is, therefore, an unmet need for a label-free technique that rapidly probes suspended individual cells. This technique will overcome the limitations of conventional approaches, such as the requirement of fluorescent-tagging and incapable of analyzing biomechanical properties of cells associated with classical flow cytometry methods.

Here, we present a label-free and high throughput flow cytometry technique by combining simultaneous UHF ultrasound backscatter and laser-induced photoacoustic waves generated from individual cells or particles in a microfluidic platform. Our technique builds upon previously combined ultrasound and photoacoustic methodology, by integrating with microfluidics, to achieve high throughput label-free analysis of single cells in heterogeneous solutions.

The main advantage of the proposed approach is that multiple biophysical parameters are acquired simultaneously, label free, from a single cell. This is achieved by integrating an ultrasound transducer to a polydimethylsiloxane (PDMS)-based microfluidic device to simultaneously detect both ultrasound and photoacoustic signals emitted from particles or cells flowing inside the microfluidic channel. Simultaneous ultrasound backscatter and photoacoustic signal acquisition provides multiple parametric analyses of the same cell. Physical and morphological properties are derived from the ultrasound backscatter and optical absorption properties from the photoacoustic waves. This approach limits the possibility of environmental and time dependent experimental variations of the cells under investigation. Moreover, by targeting distinct endogenous chromophores using different optical-excitation wavelengths (e.g. UV laser excitation to target DNA/RNA), different intracellular components can be detected, and quantified. The same methodology can be used for the characterization of extracellular vesicles (EV) and other biological specimens of interest.

Our proposed label-free multiparametric approach also has advantages in sample preparation. When analyzing blood samples using optical-based flow cytometry methods, WBCs are isolated with density gradient separation. The resulting RBCs are lysed before tagging the WBCs with fluorescent molecules. This is done to minimize the interference of signals produced by various cells in the sample. 107,108 and is important in immunophenotyping peripheral blood T-cells, as well as and their subsets, for diagnostic applications.(109) Additionally, the RBC lysis buffer increases the membrane permeability and alters the plasma membrane, damaging WBCs. RBC lysis can also cause leukocyte activation. 110 $111,112,113)$ In the proposed approach, WBCs and RBCs can easily be differentiated based on their optical absorption. Therefore, the adverse effects of buffers and reagents on the WBCs can be avoided using acoustic flow cytometry. Furthermore, our approach can avoid the use of expensive antibodies and fluorescent molecules, lowering the overall cost of the test.(114) 
As a proof-of-concept of this technique, we show that this acoustic flow cytometer can identify and count RBCs and WBCs, showing good agreement with a commercial fluorescence-based activated cell sorting (FACS) system. Further processing of the ultrasound and photoacoustic signals from the differentiated individual RBCs and WBCs has potential applications in analyzing the biomechanical and optical absorption related properties of individual cells.(43) The proposed acoustic flow cytometry approach can be used in a variety of biological applications that are based on counting, sizing, and identifying cells in a sample. One application that we are pursuing where such measurements are important is the detection of circulating tumor cells.

\subsection{Methods}

\section{Microfluidic device fabrication}

To fabricate an integrated microfluidic device, we use a 3D printed (ProJet 3510 HD, 3D systems, Rock Hill, SC, USA) transducer mold (see Appendix B, Fig. B.3p), a 3D printed alignment frame (Fig. B.3.), and a CNC machined metal mold (Fig. B.3 d). The transducer mold, which is a replica of the ultrasound transducer (Fig. B.3 ), retains the dimensions and the screw threads of the transducer. The tip of the transducer mold contains a hole of $300 \mu \mathrm{m}$ in diameter and $2 \mathrm{~mm}$ deep. The metal mold contains a $1 \mathrm{~mm}$ tall and $280 \mu \mathrm{m}$ diameter vertical pin, on the channel structure positioned next to the cross junction (Fig. B.3 d). For making the final microfluidic device, we first screw the transducer mold into the alignment frame and inserted both onto the metal mold ensuring that the pin slides into the hole. (An arrow is used to indicate the hole and the pin in Fig. B.3). The assembly then is filled with liquid PDMS and stored in an oven at $70^{\circ} \mathrm{C}$ for 3 hours. Afterwards, the cured PDMS chip along with the 3D printed molds (Fig. B.3 ) are removed from the metal mold, the transducer mold is unscrewed, and the resulting chip is bonded onto a microscope slide using oxygen plasma (Harrick Plasma, Ithaca, NY, USA). A nanoneedle is inserted into the center of the cross junction and is glued to the glass slide using two-component epoxy glue (Henkel Canada Corporation, Mississauga, ON, Canada). Finally, the ultrasound transducer is screwed into the device (Fig. B.3f). The pin-hole design on the molds automatically aligns the transducer to the channel (Fig. B.3p) and opens an interconnection between the transducer and the microfluidic channel.

\section{Ultrasonic and optical systems}

A PCI card-based trigger source triggers the ultrasound and the laser pulses. Monocycle pulses from a $300 \mathrm{MHz}$ pulser produce ultrasound pulses from a single element focus ultrasound transducer with a center frequency of $375 \mathrm{MHz}$. The focal length of the transducer is $300 \mu \mathrm{m}$ and the focal zone is an ellipsoid with lateral and axial semi-axes of $15-18 \mu \mathrm{m}$ and $5-8 \mu \mathrm{m}$, respectively. Signals detected from the transducer are high-pass filtered (>100 MHz) and amplified by $30 \mathrm{~dB}$ (Miteq, USA). Resulting signals are then acquired by a Cobramax digitizer card (Gage Applied, USA) connected to a computer. 
Laser pulses pass through a beam expander and an optical fiber before being reflected, from a combination of mirrors, towards a dichroic mirror. The dichroic mirror selectively reflects the $532 \mathrm{~nm}$ laser to a 10X optical objective that focuses the laser beam to the interrogation zone. A xiQ CCD camera (Ximea GmbH, Germany) is used to monitor inside microchannel during the experiments. A detailed flow diagram of the acoustic flow cytometry system is presented in Fig. B.4 of the Appendix B.

\section{Sample preparation}

Two different stock solutions of $3 \mu \mathrm{m}$ diameter black and white polystyrene particles (Polysciences, USA) in deionized (DI) water were prepared at a concentration of 6-7 x $10^{6}$ particles $/ \mathrm{mL}$. The particles were added to DI water and vortexed for homogeneous mixing. The stock solutions were vortexed every time before they were used.

A unit of whole blood was obtained from the Canadian Blood Services. WBCs from the whole blood are isolated using Histopaque-1077 (Sigma-Aldrich Corporation, St. Louis, MO, USA). Briefly, an equal volume of blood was used to form a double gradient over the Histopaque-1077, followed by centrifugation at $400 \mathrm{x}$ g for $30 \mathrm{~min}$ and buffy coat containing WBCs was removed and diluted in PBS solution. The isolated WBC solution contains a number of residual RBCs. The isolated solution containing both RBCs and WBCs is diluted to approximately 4-6 million cells per $\mathrm{mL}$ in PBS and is passed through the acoustic flow cytometer. From the diluted solution, we take one aliquot of $200 \mu \mathrm{L}$ that is incubated with CD45 antibodies (Human CD45, R-PE conjugate, Life Technologies, Carlsbad, CA, USA) for 15 mins, and another aliquot of $200 \mu \mathrm{L}$ that is incubated with DRAQ5 (Thermo Fisher Scientific, Waltham, MA, USA) for 30 mins. The CD45 binds only to WBCs and DRAQ5 is a supravital fluorescent dye that stains WBC nuclei. These CD45 tagged and DRAQ5 stained samples are used in the FACS (BD LSRFortessa X-20, Becton, Dickinson and Company, Franklin Lakes, NJ, USA) experiments.

\section{Signal analysis}

The signals were post processed using Matlab after the complete acquisition. During the acquisition, $\mathrm{RF}$ lines from a cell or particle were saved if the ultrasound signals were higher than $3.5 \mathrm{mV}$. Every saved signal was then analyzed to see if it contained both ultrasound and photoacoustic signals. In case of particles, if RF lines contained both the ultrasound and photoacoustic, they were counted as signals from a black bead. Alternatively, they were counted as white beads. Similarly, for blood cell experiments, the signals containing both ultrasound and photoacoustic waves were counted for RBCs, while signals with only ultrasound were counted as WBCs. 


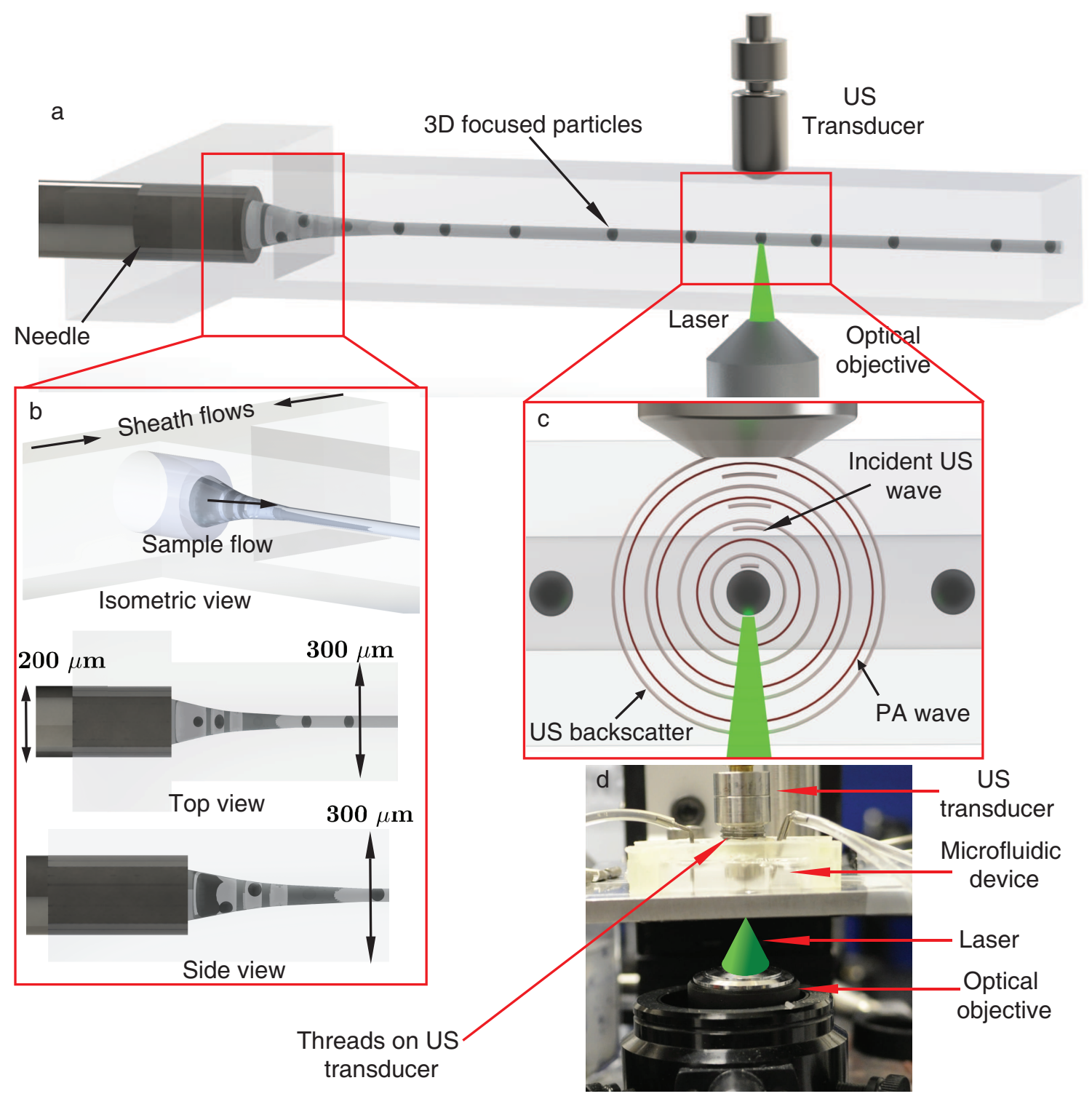

Figure 3.1: Conceptual schematic illustration of the acoustic flow cytometry system. a) The illustration shows the overall design of a microfluidic device with collinearly aligned ultrasound transducer and laser focusing optical objective. b) Hydrodynamic 3D flow focusing of a sample flow in the microfluidic device. The isometric view shows how the sample flow infused through the embedded needle at the cross-junction is 3D focused by the sheath flows from the side channels. The top and side views illustrate the position of the needle relative to the channel geometry. Gaps above, below, and on the sides of the needle allow the sheath flows to push the sample flow to the center of the channel. c) The magnified view of a collinearly aligned transducer and laser beam. The incident ultrasound (US) wave and laser beam interact with a particle at the interrogation zone producing both ultrasound backscatter and photoacoustic (PA) waves. d) Image of an experimental setup that shows how the laser is focused on the interrogation zone of the ultrasound transducer. 


\subsection{Results}

\subsubsection{Acoustic flow cytometry concept}

The acoustic flow cytometer is composed of a microfluidic device and fluid manipulation pumps; an ultrasonic system consisting of an UHF transducer, ultrasonic pulse generator, and control tools; and an optical system consisting of a laser source and optical components such as lenses, mirrors, and light sources. The conceptual illustration of the acoustic flow cytometer in Fig. 3.1 shows how the microfluidic, ultrasonic, and optical systems are integrated. In this system, a heterogeneous suspension of cells is hydrodynamically focused, and passes through the focal zone of the ultrasound beam and focal spot of the laser (i.e. interrogation zone), generating the ultrasound backscatter and photoacoustic waves.

We use a $375 \mathrm{MHz}$ single element focusing UHF ultrasound transducer (Kibero Gmbh, Germany), which has a focal length of $300 \mu \mathrm{m}$. The focal zone has an ellipsoidal geometry with major and minor axes of $15-20 \mu \mathrm{m}$ and 5-8 $\mu \mathrm{m}$, respectively. Since sample cells need to flow through this small focal zone, in a single stream, we require a precise and controlled method to focus the sample solution. To achieve this, we modify a stable two dimensional (2D) flow focusing microfluidic setup we reported previously for this application.(51) Namely, we design our PDMS (Sylgard 184 silicone elastomer kit, Dow Corning, Midland, MI, USA) based microfluidic device with a cross-junction channel architecture. Fig. 3.1p shows that at the junction, sample flow injected through an integrated nanoneedle $(\mathrm{ID}=100 \mu \mathrm{m}, \mathrm{OD}=200 \mu \mathrm{m}$, Japan Bio Products Co. Ltd., Tokyo, Japan) meets the two sheath flows. The metal mold, for patterning the microfluidic device, is made using a computer numerical control (CNC) machine resulting in $300 \mu \mathrm{m}$ deep microchannels. At the cross-junction (Fig. 3.1p, isometric view), a needle-guiding channel, two side-channels, and a downstream channel meet, which are 100, 200 and $300 \mu \mathrm{m}$ wide, respectively. This geometry enables the sheath flow to hydrodynamically focus the sample flow in both the lateral and axial directions.

Another critical aspect for our microfluidic acoustic flow cytometer is the integration and alignment of the ultrasound transducer with the focused flow in the microchannel (Fig. 3.1.). To achieve the precise alignment of the transducer, we exploit the threaded cylindrical surface of the ultrasound transducer, and mold similar threads to the transducer cavity in our microfluidic mold (see threaded transducer surface in Fig. 3.1d). After the PDMS microfluidic device is patterned, the ultrasound transducer is manually inserted into the cavity of the PDMS microfluidic device. The lateral alignment of the transducer with the focused flow is further refined by adjusting the flow rates of the sheath flows, and the axial alignment is achieved by moving the transducer in or out of the threaded cavity. We use a Pump 11 Elite syringe pump (Harvard Apparatus, Holliston, MA, USA) to infuse the sample flow (0.5-5 $\mu \mathrm{L} / \mathrm{min}$ ) and a pressure pump (CorSolutions LLC., Ithaca, NY, USA) to infuse the sheath flows (15$60 \mu \mathrm{L} / \mathrm{min}$ ). We use polystyrene microparticles (Polysciences Inc., Warrington, photoacoustic, USA) for alignment experiments because they produce strong backscatter signals, and we adjust the sheath flow and transducer axial position to achieve maximum backscattered amplitude signals, indicating good 
alignment. For photoacoustic signals, the laser spot is focused by adjusting the device using a mechanical X, Y, Z-stage to ensure that the transducer and the laser foci are collinear (Fig. 3.1 a).

\subsubsection{Generation of ultrasound backscatter and photoacoustic signals}

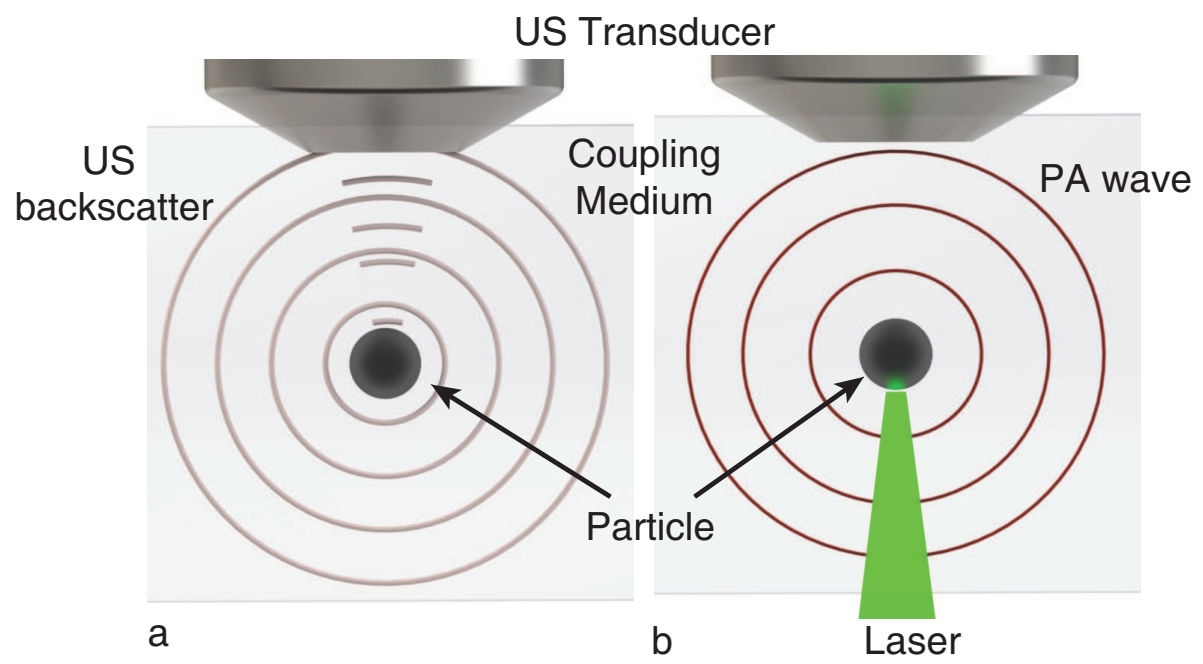

Figure 3.2: (a) the ultrasound (US) backscatter and (b) the photoacoustic (PA) waves that are generated by a particle located in the focal zone of the transducer, as a result of the ultrasound and laser pulses that interact with the particle, respectively.

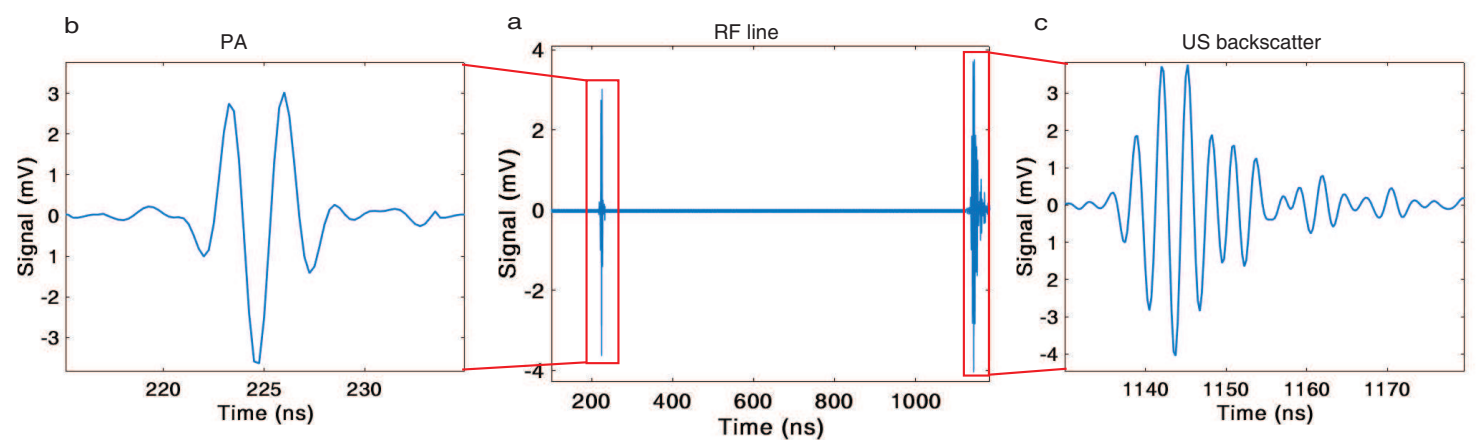

Figure 3.3: (a) A representative RF line detected and recorded by the ultrasound (US) transducer from a $3 \mu \mathrm{m}$ diameter black polystyrene particle. The ultrasound and photoacoustic (PA) signals in the RF line are highlighted in the red boxes. Transducer artifacts are removed from the signal to emphasize the detected ultrasound backscatter and photoacoustic waves. Measured ultrasound backscatter (c) and photoacoustic (b) signals from the individual particle. These signals are gated using hamming window from the RF line. 

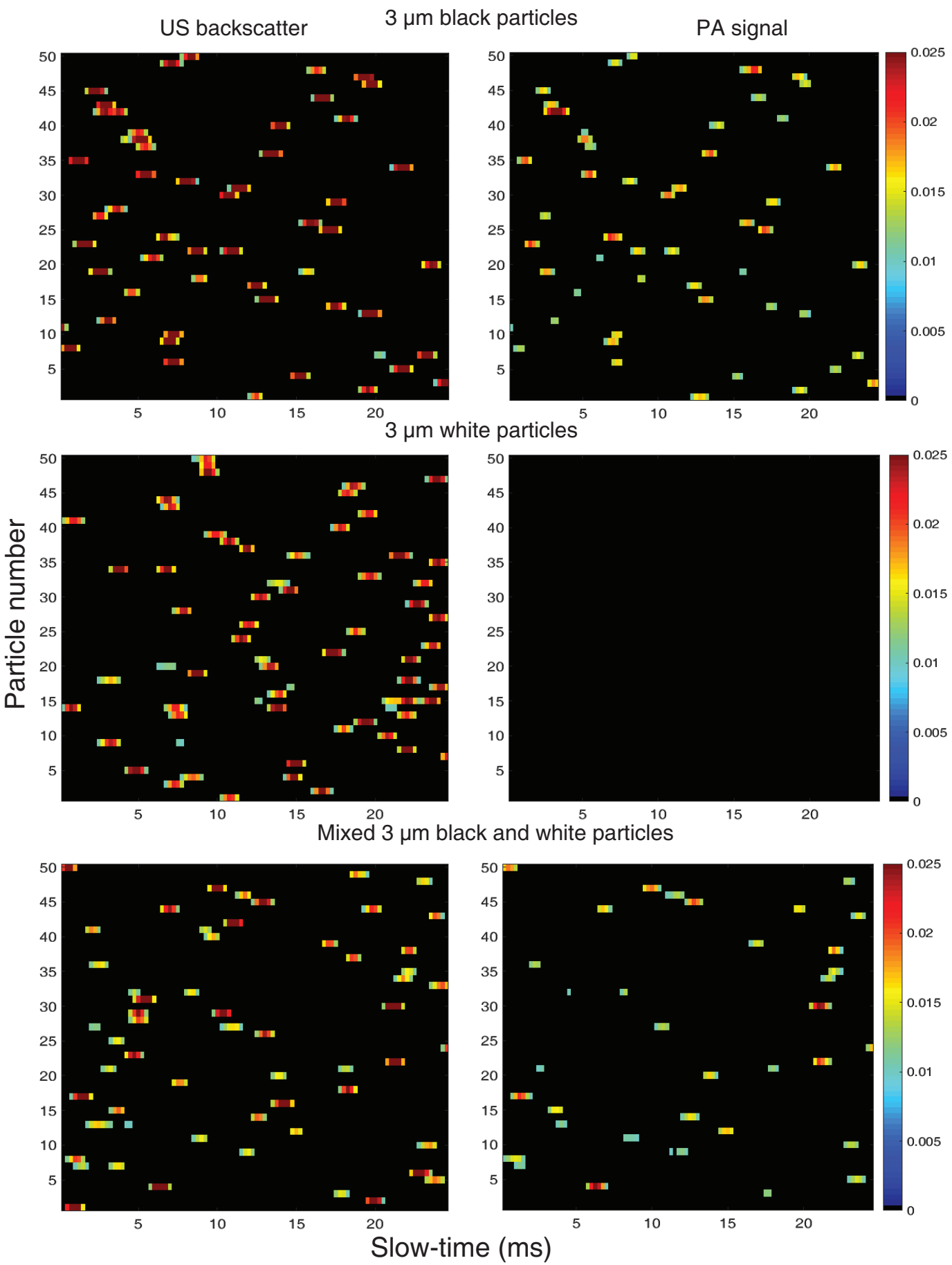

Figure 3.4: Maximum amplitude plot of the time domain signals of the ultrasound (US) backscatter and photoacoustic (PA) waves detected by the transducer for three different samples: black particles exclusively (top row), white particles exclusively (middle row), and a mixture of both types of particles (bottom row). The $y$-axes represent 50 particles detected by the acoustic flow cytometer from each experiment. The $x$-axes represent the "slow-time" of the acquisition by the transducer while the particles flow downstream. The amplitude of the detected signals (in mv) is represented by color bars. 
Fig. 3.2 shows schematic illustrations of the ultrasound backscatter and photoacoustic waves generated by a particle located in the focal zone of the transducer. An incident ultrasound pulse scattered by the particle is detected as ultrasound backscatter (Fig. 3.2 ). Similarly, the optical energy absorbed by the particle generates photoacoustic waves (Fig. $3.2 \mathrm{p}$ ). In our device, the laser and ultrasound transducer are triggered simultaneously. The speed of light is much faster than the speed of sound, thus the laser excites the particle first, generating a photoacoustic wave. Approximately $900 \mathrm{~ns}$ later, the ultrasound pulse scatters off the particle. The photoacoustic wave is detected first while the reflected ultrasound is detected by a short delay. Fig. 3.3 shows a typical RF line measured from a $3 \mu \mathrm{m}$ black polystyrene bead (processed so that the transducer internal reflections are subtracted). To analyze the detected signals, the individual photoacoustic and ultrasound signals are isolated using a Hamming window and saved for further processing.

\subsubsection{Calibration of the acoustic flow system}

Black polystyrene particles have high optical absorption, in contrast to white particles. Hence, black particles produce both ultrasound and photoacoustic waves, while white particles produce only ultrasound backscatter.

For each particle detected by the system, we record one hundred subsequent RF lines at pulse repetition frequency $4 \mathrm{KHz}$ (referred as the slow-time) and each signal is sampled at $4 \mathrm{GHz}$ sampling rate (referred as a fast-time). The concept of fast and slow-time is shown in Appendix B, Fig. B.1. As the particles pass through the interrogation zone, RF lines containing the ultrasound and photoacoustic signals are recorded as the particles flow through the channel. Fig. 3.4 plots of the maximum amplitude of the ultrasound backscatter (left column) and photoacoustic (right column) signals detected from individual experiments using black particles exclusively (top row), white particles exclusively (middle row), and a mixture of both black and white particles (bottom row). Color intensity represents the maximum amplitude of the respective signals. The $y$-axis represents the number of consecutive acquisitions for which a signal (whether ultrasound or photoacoustic) is detected. The $x$-axis shows the slow-time of signal acquisition. The results from the experiments show that for particles (both black and white) passing through the interrogation zone for which an ultrasound backscatter signal was detected, only black particles produce photoacoustic signals. The top row in Fig. 3.4 demonstrates that almost 100 $\%$ of the detected black beads produce both the ultrasound and photoacoustic signals. The middle row (white beads) contains only ultrasound signals, and the bottom row (mixture) contains ultrasound and some photoacoustic signals.

\subsubsection{Comparison with Fluorescence Activated Cell Sorting (FACS)}




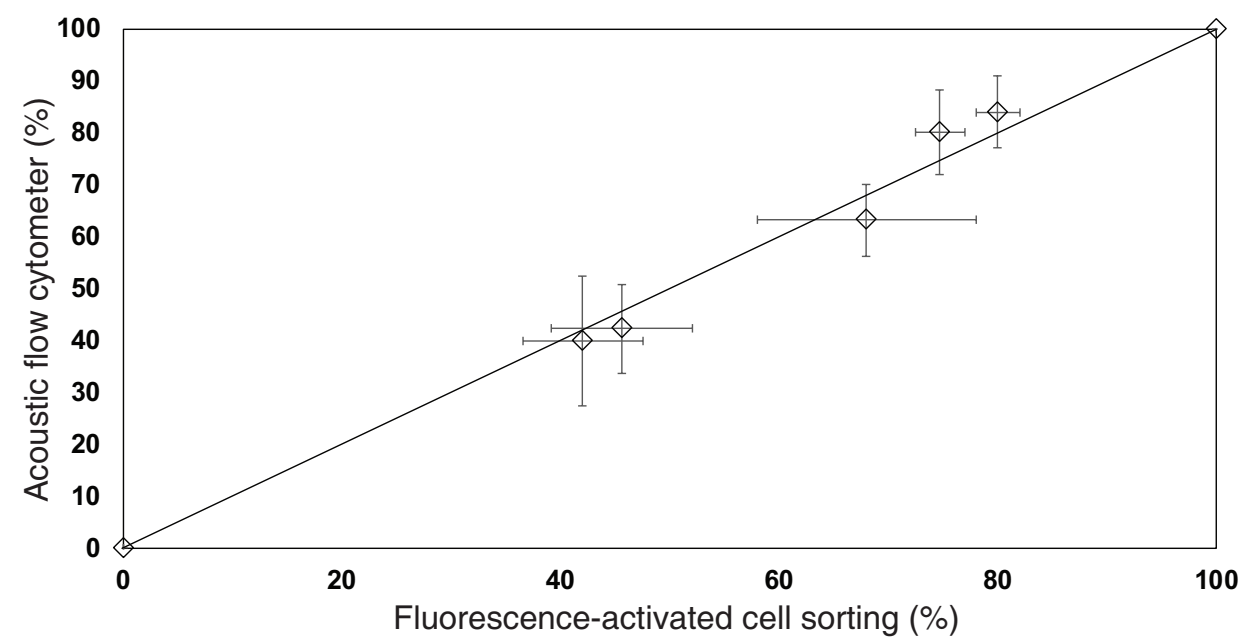

Figure 3.5: A comparison plot of the percentage of black particles in sample solutions of a mixture of black and white particles detected by the acoustic flow cytometer and the FACS. Results from the acoustic flow cytometer are comparable with those from the FACS. Error bars represent one standard deviation with data from five independent experiments. The straight line is the line of equality for both techniques.

We compare results from the acoustic flow cytometer to those from a FACS using the same sample solution of $3 \mu \mathrm{m}$ polystyrene black and white particles. The auto-fluorescence signal produced by white particles is significantly less than the signals from black particles in the APC and PE-Cy5 channels. The autofluorescence information is used to differentiate the particles. See the Appendix B (Fig. B.2) for more detail information about the autofluorescence of the polystyrene particles. To compare the results from both cytometry systems, we use mixtures of the black and white particles at five different volume ratios taken from stock solutions of black and white particles. For each volume ratio, we perform five experiments in both the acoustic flow cytometer and FACS. The results of five experiments of each sample solution in both systems are averaged and plotted in the graph shown in Fig. 3.5.

The $y$-axis represents the percentage of the black particles in the solution detected by the acoustic flow cytometer whereas the $x$-axis is the percentage of black particles detected by the FACS. The close proximity of the data points to the solid straight line in the graph shows that the measurements of the acoustic flow cytometer are in close agreement with those from FACS $\left(R^{2}=0.9881\right)$. Error bars on each point in $x$-direction and $y$-direction represent one standard deviation from the five experiments on FACS and acoustic flow cytometer, respectively. For each sample, we measure at least 500 particles using the acoustic system and 10,000 particles using the FACS. The data points at $0 \%$ and $100 \%$ represent the samples with only white particles and black particles, respectively. 


\subsubsection{Label-free blood cell differentiation}

We experiment with human blood to demonstrate the acoustic flow cytometer's ability to differentiate between red and white blood cells in a label-free manner. RBCs absorb light at $532 \mathrm{~nm}$, generating strong photoacoustic signals, whereas WBCs do not significantly absorb light at this wavelength.(115) Therefore, WBCs do not generate detectable photoacoustic signals. Individual RBCs produce both ultrasound backscatter and photoacoustic signals, whereas WBCs produce only ultrasound backscatterthis is the principle with which the acoustic flow cytometer differentiates between WBCs and RBCs.
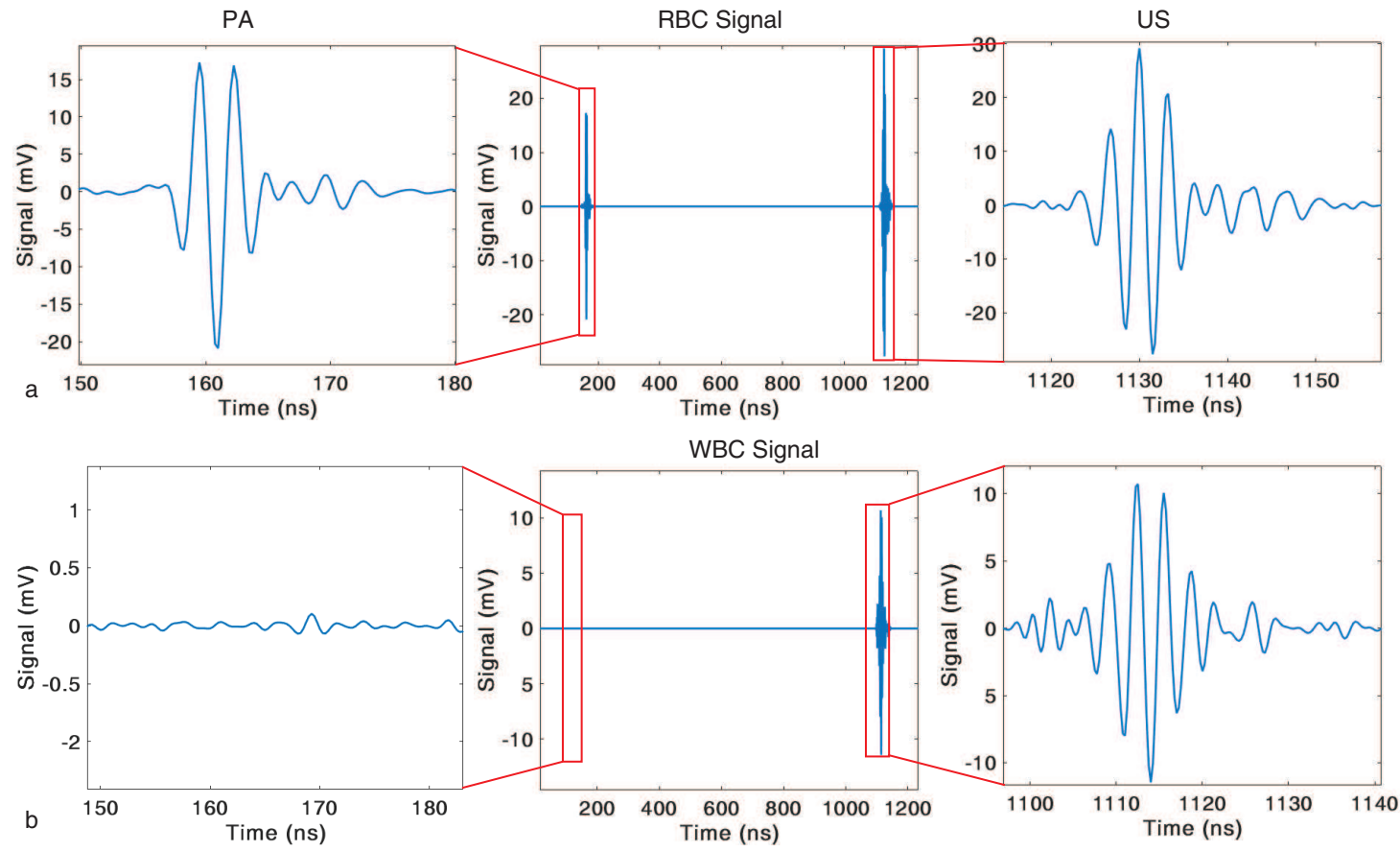

Figure 3.6: Representative signals detected by the transducer for a) RBC and b) WBC samples. The signal from an RBC contains both ultrasound (US) backscatter and photoacoustic (PA) waves whereas signals from WBC contains only ultrasound backscatter.

We use sample solutions of isolated WBCs. These sample solutions contain various concentrations of residual RBCs along with the isolated WBCs without platelets. By evaluating the presence or absence of a photoacoustic signal, we classify the source of the signal as either a RBC (i.e. when ultrasound and photoacoustic signals are both present) or a WBC (i.e. when only ultrasound signals are present). Using all the RF lines with signals detected, we calculate the percentage of RBCs and WBCs present in the sample solutions. Representative experimental RF lines from both individual RBC and WBC are shown in the Fig. 3.6a and b, respectively.

We compare these results to those obtained with a FACS. Here, we separately incubate two aliquots 
of $200 \mu \mathrm{L}$ of each sample solution with DRAQ5 fluorescent dye and R-PE conjugated CD45 antibodies to label the cells. The DRAQ5 stain the nuclei of the WBCs while the CD45 antibodies attach to the membrane of the WBCs in the solutions, whereas neither DRAQ5 nor CD45 interact with RBCs. We dilute these solutions in phosphate-buffered saline (PBS) solution before introducing them into the FACS. Based on the detected fluorescence signals from the sample solutions, we calculate the percentage of RBCs and WBCs from both experiments.

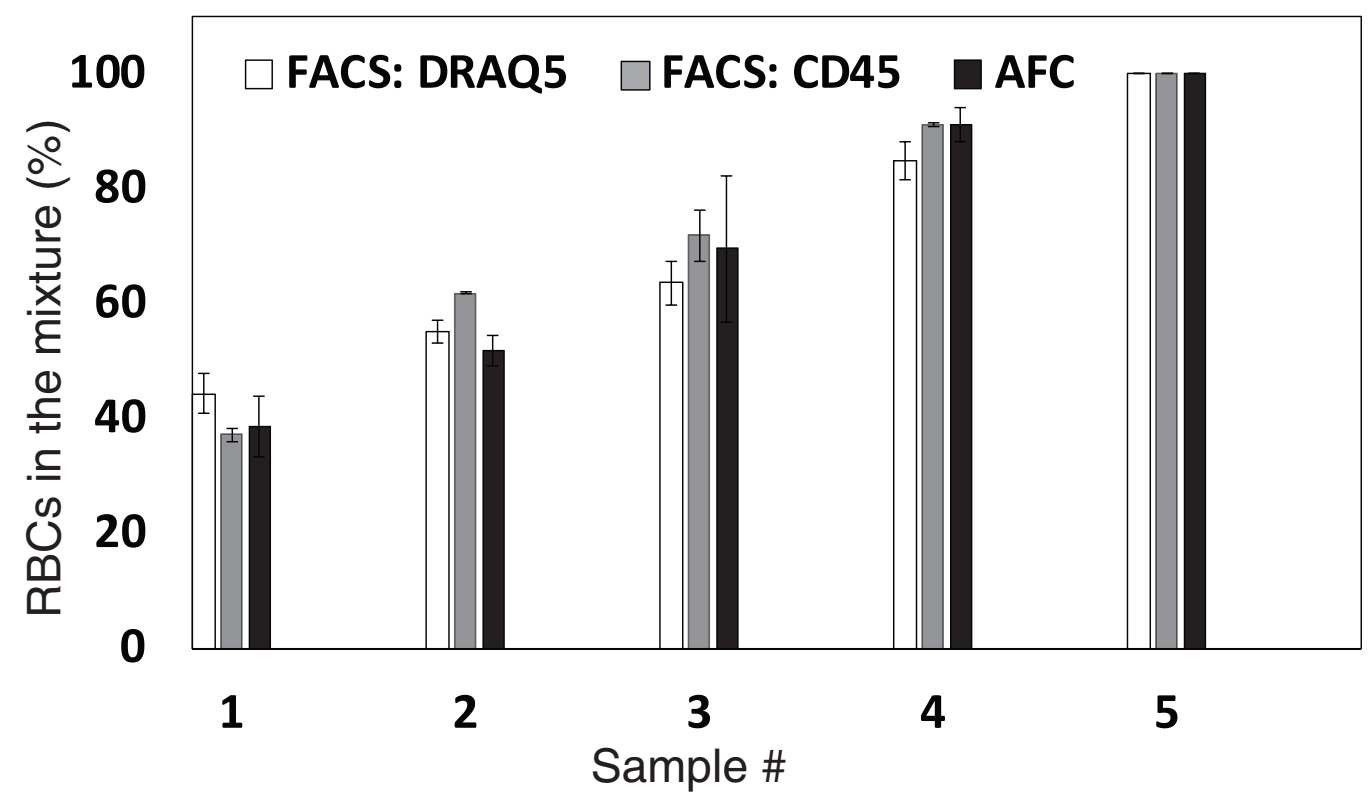

Figure 3.7: Plot showing the percentage of RBCs in mixtures of RBCs and WBCs from five sample solutions containing WBCs and RBCs using the acoustic flow cytometer (labeled AFC) and the FACS. The $y$-axis represents the percentage of the RBCs present in the sample solutions. For each experiment, the sample solutions are divided to three different volumes. The first volume is label-free, the second is incubated with DRAQ5 fluorescent dye, and the third is incubated with R-PE conjugated CD45. DRAQ5 stains WBC nuclei and CD45 bonds to the WBC membrane. The label-free solution is measured using the acoustic flow cytometer, and the other two are measured using the FACS. Error bars represent one standard deviation from the mean for three experiments.

We experiment using five different sample solutions, each containing different concentrations of RBCs and WBCs, to compare the acoustic flow cytometer and the FACS. The sample numbers are shown on the $x$-axis in Fig. 3.7 and the $y$-axis shows the measured percentage of RBCs. We test each sample three times and the average concentration for each cell type is plotted on the $y$-axis. The bars with horizontal lines represent results from label-free acoustic flow cytometry, diamond crossed bars show measurements from CD45 tagged WBCs using the FACS (FACS:CD45), and bars with solid dots show data from nuclei stained WBCs using the FACS (FACS:DRAQ5). For each acoustic flow cytometer experiment, we measure at least 500 individual cells while for each FACS experiment, we measure approximately 10,000 
cells. Error bars represent the standard deviation of the mean for three experiments. This figure shows a good agreement between the results obtained from the acoustic flow cytometer and the FACS.

\subsection{Discussion}

In summary, we demonstrate a label-free cytometry technique based on acoustic waves to analyze individual cells in a heterogeneous population. Cells are simultaneously probed by UHF ultrasound, with a center frequency of $375 \mathrm{MHz}$, and a $532 \mathrm{~nm}$ nanosecond laser. We show that the acoustic flow cytometer is able to differentiate between WBCs and RBCs in a label-free manner, and the measurements show a strong agreement with the results obtained from the FACS using labeled samples. To the best of our knowledge, this is the first demonstration of using ultrasound backscatter and photoacoustic waves in a high throughput microfluidic platform for the flow cytometry of individual cells. The acoustic flow cytometer has a throughput of 150 cells per minute, which can be further improved in the future by optimizing the flow system and increasing the pulse repetition frequency of ultrasound and laser pulses.

For a flow cytometer, a precise control and focusing of the sample particles is crucial. For our flow cytometry system, we developed a needle-based flow focusing technique, which provides a stable and precise 3D hydrodynamic focusing of sample flow where suspended individual particles and cells flow in a single stream through the interrogation zone. The 3D flow focusing is important to maintain a gap between the sample cell and the surface of the glass substrate. This separation avoids the overlap of the signals between ultrasound backscatter from the cell and ultrasound reflection from the substrate. In addition, this simple 3D flow focusing technique developed using a single-layered channel dimension can be used as an alternative for microfluidic applications, such as droplet generation.(116)

The ultrasound backscatter and photoacoustic signals detected simultaneously from individual cells can be used to extract information regarding the physical properties of cells, such as size, and shape and the optical absorption properties of the cell or its intracellular components. The optical absorption properties of the cell components can be correlated with functional properties of cells. In RBCs we can estimate the oxygen saturation or hemoglobin concentration in an individual cell. Analysis of the RF signals (Figs. 3.3 3.6 can also provide information related to the structure of the structures ultrasound, and the structures of the optical absorbers $(42,106)$ With these applications, we believe that the acoustic flow cytometry technique can provide unique advantages compared to optical-based techniques. Furthermore, this acoustic flow cytometry may find utility in applications where being labelfree is a crucial requirement, such as, identification of different cell types and circulating tumor cells and in vivo cell analysis. 


\subsection{Conclusion}

We develop a label-free acoustics based microfluidic flow cytometer based on simultaneous ultrasound backscatter and photoacoustic waves detected from individual cells or particles flowing through a microfluidic channel. The sample flow is hydrodynamically focused at a cross junction microchannel when flowing through a nanoneedle in a microfluidic device. We use a very high-frequency ultrasound backscatter (center frequency $375 \mathrm{MHz}$ ) and $532 \mathrm{~nm}$ pulsed laser-induced photoacoustic signals to analyze individual cells.

We calibrate the system using $3 \mu \mathrm{m}$ polystyrene particles. Our system is capable of differentiating the particles of various colors using the absorption property of the particles. The results obtained from acoustic flow cytometer is in agreement with those obtained from the FACS. In addition, we also show that our system is capable of label-free differentiation of RBCs from WBCs, and the measurements from the system are consistent with the results from FACS. This acoustic flow cytometer has potential application in label-free cytometry to count, size, and identify individual cells from a cell population. It may also find future utility in the detection of circulating tumor cells and performing single cell characterization of flowing cells for diagnostic applications using blood or other bodily fluids. 



\section{Chapter 4}

\section{Microfluidic generation and shrinkage of microbubbles using vacuum pressure}

The work presented in this chapter is based on the following article published in a peer reviewed journal Soft Matter. This paper was highlighted on the cover page of the journal, as a research highlight in Chemistry World magazine, and various university-based media.

Gnyawali, V.*, Moon, B.-U.*, Kieda, J., Karshafian, R., Kolios, M. C., and Tsai, S. S. H. (2017). Honey, I shrunk the bubbles: microfluidic vacum shrinkage of lipid-stabilized microbubbles, Soft Matter, 13(22), 4011-4016. https://doi.org/10.1039/C7SM00128B

\section{Author's contribution}

The bubble shrinking idea was developed together with a previous postdoctoral researcher (Dr. Byeong-Ui Moon, co-first author of the paper). The original experiment was done by Dr. Moon, with the follow-up device development, experimental plan, experimental setup design, execution of the planned experiments, and data analysis done by the author. In this research, an undergraduate research assistant, Jennifer Kieda, helped the author to collect and analyze the data.

\subsection{Introduction}

Microbubbles are used in a variety of clinical applications, ranging from imaging, diagnostics, to therapeutics. $11 ; 12 ; 13$ Microbubbles in these applications are generally injected intravenously, where 
CHAPTER 4. MICROFLUIDIC GENERATION AND SHRINKAGE OF MICROBUBBLES USING VACUUM PRESSURE

they flow in the blood stream and circulate throughout the body.(13)

In ultrasound imaging applications, microbubbles exposed to low amplitude ultrasound pulses undergo linear and non-linear oscillations, emitting strong ultrasound echo signals.(13) Signals from the echos increase the image contrast, thereby improving the visual distinction between blood and the surrounding tissues.(117) This improvement leads to increased detection sensitivity, and accuracy of imaging, facilitating enhanced detection of thrombosis and diseased tissues. $(118,119,120)$

Ultrasound and microbubbles have also been utilized for therapeutics such as in enhancing gene and drug delivery.(121, 57) Delivery occurs through pores on the membrane of tumor cells to lyse the cells or

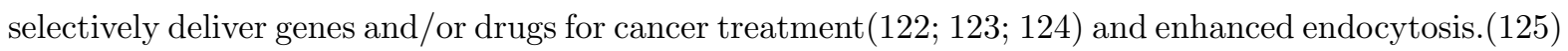

The acoustic response of microbubbles depends on the microbubbles' characteristics such as their size and shell-type, in addition to the degree of ultrasound exposure. Therefore, monodisperse (uniform characteristics) microbubbles are more desirable than polydisperse (non-uniform characteristics) microbubbles in many biomedical applications. Sub-micron control of bubble production has also the potential of generating nanobubbles (typically $550 \mathrm{~nm}$ or less), a burgeoning field in ultrasound contrast and therapy. (126) Both microbubbles and nanobubbles can also be used therapeutically,(127) and control over size is an important factor in their efficacy as microbubble behavior critically depends on size.(128) However, producing monodisperse microbubbles that have the length-scales relevant to biomedical ultrasound remains challenging.

In the aforementioned applications, the microbubbles required are typically 1-7 $\mu \mathrm{m}$ in diameter. 118 . 15) Conventional methods used to generate these microbubbles including sonification, (129) high shear emulsification, (130) inkjet printing, (131) and coaxial electrohydrodynamic atomization (CEHDA), (132) create polydisperse microbubbles at bubble diameters below $10 \mu \mathrm{m} .15,132,129)$ As a result of the microbubbles' polydispersity, filtration steps are needed to attain microbubbles of the desired size. Inkjet printing of microbubbles achieves better control of microbubble sizes, but the approach is limited to generating liquid or volatile solvent filled bubbles. (15, 129) The commercially available and U.S. Food and Drug Administration (FDA) approved DEFINITY ${ }^{\circledR}$ microbubbles are polydisperse microbubbles with an average diameter of $1-3 \mu \mathrm{m}$, but some bubbles can be as large as $20 \mu \mathrm{m}$ in diameter.(16)

While microfluidic techniques produce monodisperse microbubbles with excellent size-control, 15,16 , 17, 133 , 134 microfluidics-generated microbubbles have lower limits of size that directly depend on the dimensions of the bubble-generating microchannel orifice.(16) Generating microbubbles that are on the relevant length-scale for biomedical ultrasound and therapeutics applications either requires complexto-manufacture phase-change nanoparticles (135) or microfluidic orifice widths that are on the order of just a few micrometers. Fabricating microfluidic molds with such small orifices is expensive and requires high-resolution photolithography.(15) This makes a simple and low-cost alternative microfluidic approach that produces small microbubbles highly desirable. 
In this chapter, we describe a simple approach to shrink bubbles generated from a conventional microfluidic flow focusing orifice into microbubbles that are on the relevant length-scale for ultrasound and therapeutics applications. The novelty of our approach is in our embedded vacuum microchannels adjacent to the main liquid-filled microchannel that microbubbles flow through.(136) By tuning a single parameter, the vacuum pressure in the adjacent microchannels, we controllably shrink microbubbles in the main liquid channel to as small as sub-micrometer diameters. We anticipate that the simplicity of our approach will make it potentially useful to the biomedical ultrasound community, and also open up new avenues of applications for microbubbles.

\subsection{Methods}

We use air as the dispersed gaseous phase. The continuous liquid phase is a mixture of lipids, glycerol (Sigma Aldrich Corporation, St. Louis, MO, USA), and pluronic F-68 (Fisher Scientific, Pittsburgh, PA, USA) in a 1:1:1 volumetric ratio. The lipid solution is prepared using 9:1 molar ratio of 1,2-distearoylsn-glycero-3-phosphocoline (DSPC) (Avanti Polar Lipids, Alabaster, AL, USA) and 1,2-distearoyl- snglycero-3-phosphoethanolamine-N [methoxy-(polyethylene glycol)-5000] (DSPE-PEG5000) (Avanti Polar Lipids, Alabaster, AL, USA) in saline (lipid concentration of $1.5 \mathrm{mg} / \mathrm{mL}$ ). The composition of the

lipids is similar to the FDA-approved and commercially available DEFINITY ${ }^{\circledR}$ microbubbles.(137) The interfacial tension of the continuous phase mixture and air is $1.5 \mathrm{mN} / \mathrm{m}$, measured using the pendant drop method.(138) We note that this continuous phase mixture is also used in the medical ultrasound literature to stabilize perfluorocarbon droplets, which are vaporized in animal models for potential applications in contrast-enhanced imaging and drug delivery.(139) 


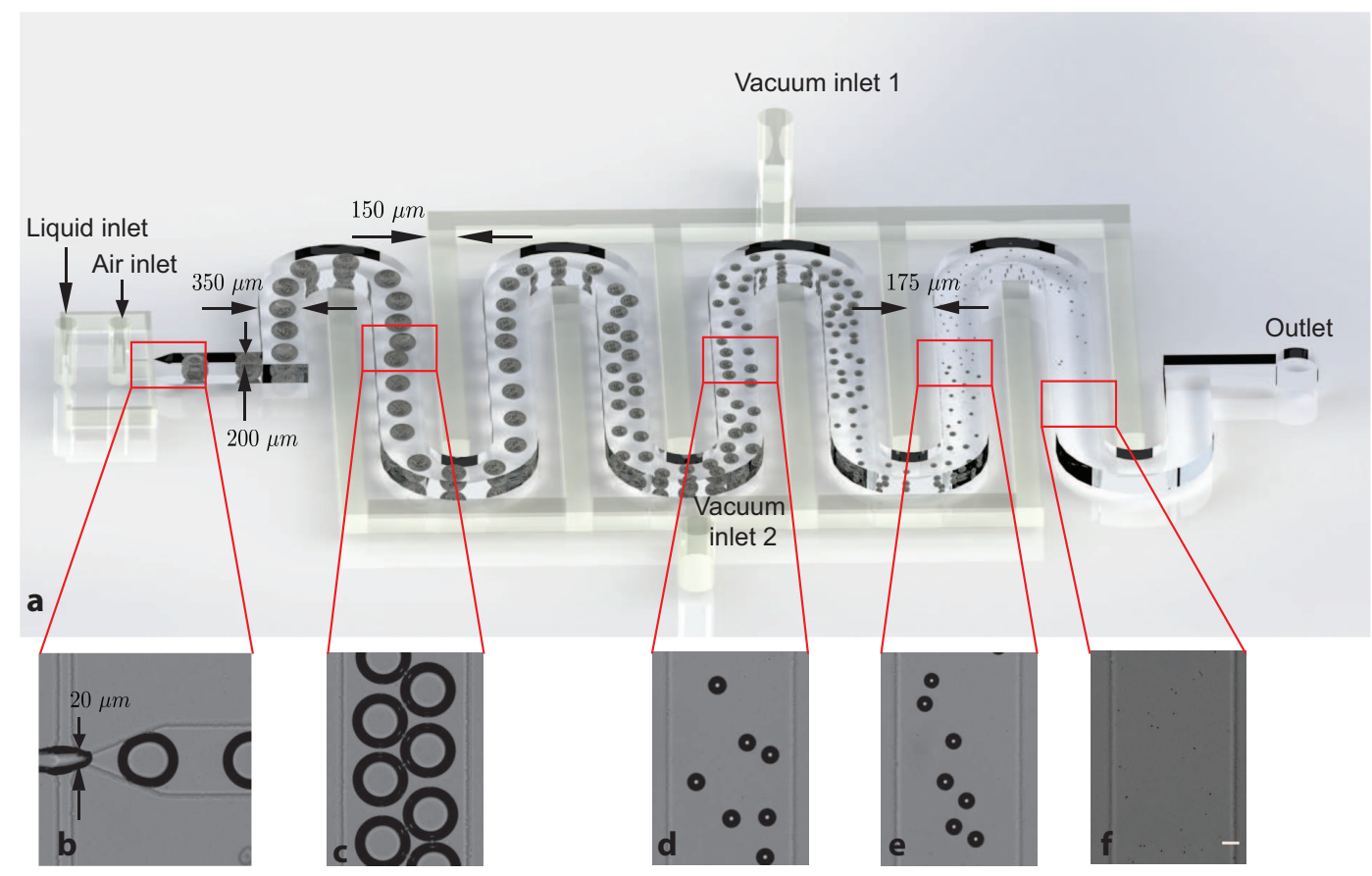

Figure 4.1: Our microfluidic device for microbubble generation and shrinking. (a) The three dimensional (3D) schematic diagram shows that bubbles are (b) generated at the orifice and (c-f) shrink as they flow downstream in the serpentine microchannel. Two sets of vacuum channels are embedded adjacent to the serpentine channel. Vacuum inlets 1 and 2 connect the vacuum source to the vacuum microchannels. Representative experimental images (b - f) show the sequential shrinking of the microbubbles in the microchannel. These experimental images are taken from an experiment where the vacuum pressure, $P_{v}$ $=-50 \mathrm{kPa}$. Scale bar represents $50 \mu \mathrm{m}$.

We pattern a single-layer PDMS (Sylgard 184 silicone elastomer kit, Dow Corning, Midland, MI, USA) slab using conventional soft lithography techniques. (80) Briefly, on a silicon wafer, we spin-coat a $80 \mu \mathrm{m}$ thick film of SU-8 2075, which is then patterned by ultraviolet (UV) light through a photomask that is designed with a computer-aided design (CAD) software (AutoCAD 2010, Autodesk, Inc., Dan Rafael, CA, USA) and printed on a transparency sheet (25 400 dpi, CAD/ART Services Inc., Bandon, OR, USA). The pattern formed on the wafer by photolithography is transferred to the PDMS. Inlets for air, lipid solution, and vacuum, and the outlet, on the PDMS slab are opened using a $1 \mathrm{~mm}$ diameter biopsy punch (Integra Miltex, Inc., Rietheim-Weilheim, Germany). The PDMS slab is then irreversibly bonded to a glass microscope slide using oxygen plasma (Harrick Plasma, Ithaca, NY, USA) to complete the microfluidic chip (Fig. 4.1)).

Fig. 4.1 b shows the flow focusing orifice with a width of $20 \mu \mathrm{m}$. The channel immediately downstream of the orifice has a width of $200 \mu \mathrm{m}$ (Fig. 4.1 ). Even further downstream, the serpentine channel has a width of $350 \mu \mathrm{m}$ and a total length of $350 \mathrm{~mm}$. Two vacuum inlets on the two sides of the device supply vacuum pressure to the microfluidic device via interdigitated microchannels that are patterned 
adjacent to the serpentine channel (Fig. 4.17). The interdigitated vacuum channels have widths of 150 $\mu \mathrm{m}$ and are separated by a distance of $175 \mu \mathrm{m}$ from the serpentine channel. All channels have height $h=80 \mu \mathrm{m}$.

We supply pressurized air to the air inlet of the device through a control valve using Tygon tubing (Saint gobain S.A., Courbevoie, France). The control valve (Omega Engineering Inc., Norwalk, Connecticut, USA) is coupled to a pressure gauge (Omega Engineering Inc., Norwalk, Connecticut, USA) to control the air pressure at the air inlet. We use an inlet air pressure, $P_{a}=4 \mathrm{psi}$, in all of our experiments. The continuous liquid phase containing the lipid solution is supplied by a constant flow rate syringe pump (Harvard Instruments, Holliston, MA, USA) at a flow rate, $\mathrm{Q}=4 \mu \mathrm{L} / \mathrm{min}$. Microbubbles pinch-off at the flow focusing orifice (Fig. $4.1 \mathrm{~b}$ ), and flow into the serpentine section of the microchannel, where the vacuum pressure from the vacuum microchannels cause the microbubbles to shrink continuously until they reach the outlet (Figs. 4.1. - 4.1F). In our experiments, we tune the vacuum pressure $P_{v}=0$ to -90 $\mathrm{kPa}$. We use a Mityvac hand vacuum pump (Mityvac, St. Louis, MO, USA) with an integrated pressure gauge to supply the vacuum pressure, and connect the pump to the interdigitated vacuum microchannels using Tygon tubing. A flow diagram of this experimental setup is available in Appendix C.

We use an inverted microscope (Olympus Corp., Tokyo, Japan) and an attached high speed camera (Phantom M110, Vision Research, Wayne, NJ, USA) to capture experimental images of the microbubbles. The camera operates at $100 \mathrm{fps}$ with an exposure time of $500 \mu \mathrm{s}$. Using ImageJ software, we measure the initial microbubble diameter, $D_{i}$, immediately after they are generated at the orifice (Fig. 4.1 ). Subsequently, we measure the final microbubble diameter, $D_{f}$, at a fixed location in the serpentine microchannel near the outlet of the device (Fig. 4.1).

\subsection{Discussion}

The microbubbles generated at the orifice have initial diameters, $D_{i}$, that are larger than the microchannel height $h=80 \mu \mathrm{m}$, which causes the microbubbles to be confined to a discoid shape. However, the vacuum-shrunk microbubbles near the outlet of the microfluidic device have diameters $D_{f}<h$, resulting in unconfined spherical microbubbles. To make an equivalent comparison of initial and final microbubble sizes, we convert our measurements to the initial and final volumes of the microbubbles, $V_{i}$ and $V_{f}$, respectively, to determine the amount of microbubble shrinkage. See Appendix C for details about the conversion (which are based on equations we used in a recent paper.(140))

Figs. 4.1p-4.1: show representative experimental images of microbubbles at different locations in the serpentine microfluidic channel downstream of the bubble generating orifice. The microbubbles shrink as they flow downstream towards the outlet. These images are taken from an experiment where the applied vacuum pressure is $P_{v}=-50 \mathrm{kPa}$. A representative video of the microbubble shrinkage process 


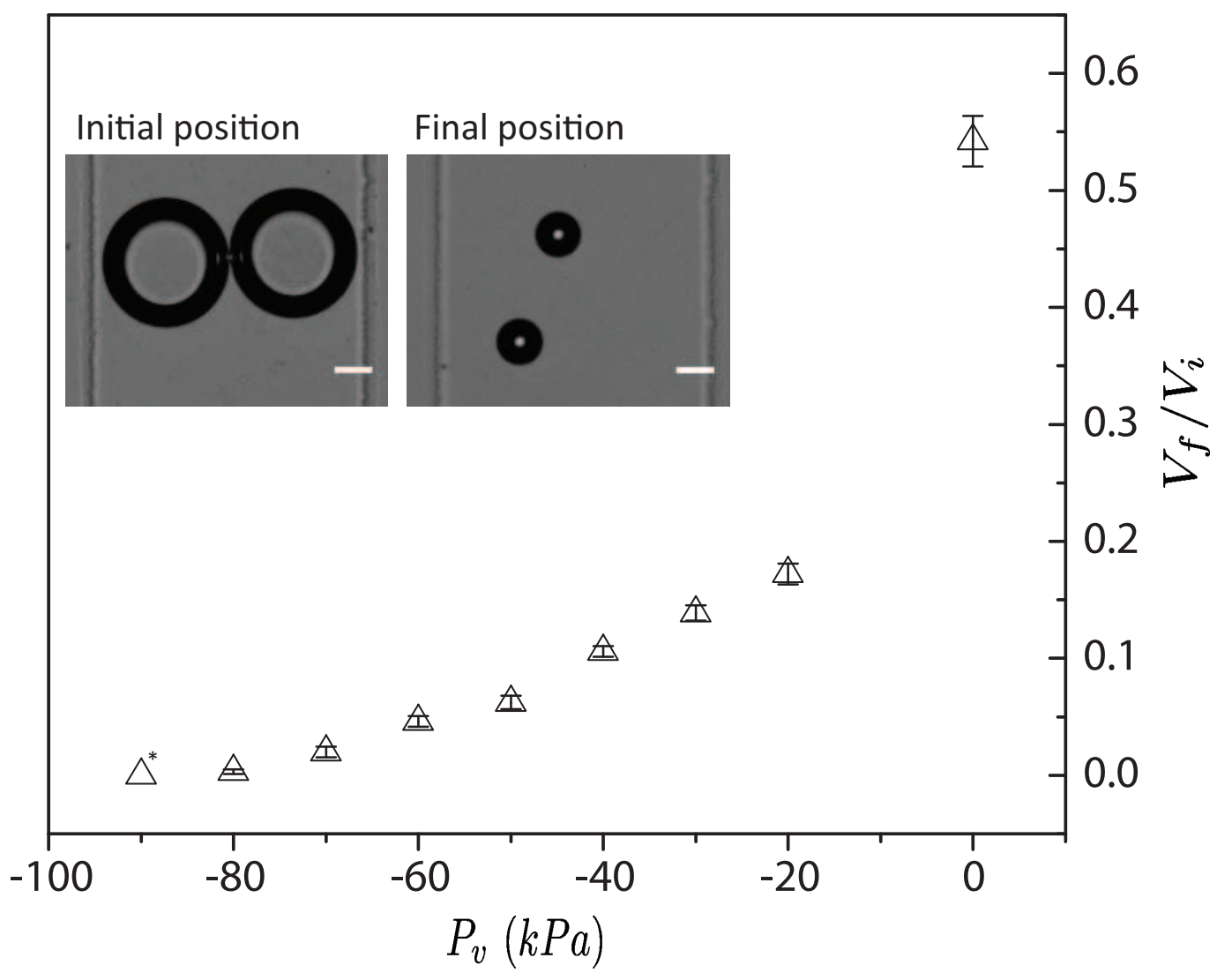

Figure 4.2: A plot of the normalized final microbubble volume $\left(V_{f} / V_{i}\right)$ versus the applied vacuum channel pressure, $P_{v}$, in the vacuum microchannels. These experimental results show that, even in the absence of an applied vacuum pressure, the microbubbles shrink to approximately $55 \%$ of their initial volume when flowing through the long serpentine channel. When a vacuum pressure is applied to the vacuum microchannels, we observe that the microbubbles in the serpentine microchannel shrink more dramatically, and their shrinkage increases monotonically with increasing magnitude of the applied vacuum pressure. Here, error bars represent one standard deviation of 10 samples. The insets show representative experimental images of microbubbles at the initial and final positions in the serpentine microchannel, corresponding to microbubble volumes $V_{i}$ and $V_{f}$, respectively. The datum indicated by "*" corresponds to the final microbubble volume $V_{f} / V_{i}$ from the experiment where the vacuum pressure is $P_{v}=-90 \mathrm{kPa}$. Here, the microbubbles are not visible under the microscope, so we define their volume $V_{f} / V_{i}=0$. The inset images are from an experiment where the vacuum pressure $P_{v}=-50 \mathrm{kPa}$. Scale bars represent $50 \mu \mathrm{m}$. 
CHAPTER 4. MICROFLUIDIC GENERATION AND SHRINKAGE OF MICROBUBBLES USING VACUUM PRESSURE

is available at http://www.rsc.org/suppdata/c7/sm/c7sm00128b/c7sm00128b1.avi.

Fig. 4.1p shows the orifice where the bubbles are initially generated in the microchannel. The size of these bubbles are known to depend on the size of the orifice, inlet air pressure, liquid-air surface tension, and the continuous liquid flowrate.(16) In our experiments, the orifice size, inlet air pressure, liquid-air surface tension, and continuous liquid flow rate are all held constant as described above. Therefore, the initial microbubble volume, $V_{i}=1.5 \mathrm{~nL}$, which we measure immediately downstream of the orifice, is approximately the same in all of our experiments.

Fig. 4.2 shows a plot of the normalized final microbubble volume, $V_{f} / V_{i}$, versus the applied vacuum pressure, $P_{v}$, in the vacuum microchannels. Here, the final microbubble volume, $V_{f}$, measured at a fixed location near the outlet for all experiments in the serpentine microchannel, is normalized by the initial volume, $V_{i}$, which is measured immediately downstream of the bubble-generating orifice. The inset of Fig. 4.2 shows two representative images of the microbubbles at the initial and final measurement locations, corresponding to initial and final volumes $V_{i}$ and $V_{f}$, respectively.

We observe that when the applied vacuum pressure, $P_{v}=0$, the final microbubble volume $V_{f}$ is approximately $55 \%$ of the initial microbubble volume, $V_{i}$. We hypothesize that this microbubble shrinking effect, in the absence of an applied vacuum pressure, is due to the high pressure of the gas and liquid in the microchannel, which is a result of the pressure-driven nature of the flows. The pressure in the microchannel is higher than atmospheric pressure, so according to Henry's law, the molecular components of air in the microbubbles will become more soluble in the continuous liquid. This observation of shrinking microbubbles is consistent with the observation of Cubaud et al who find that carbon dioxide microbubble diameters decrease by as much as $55 \%$ in a $10 \mathrm{~cm}$ long serpentine microchannel.(141) Convective liquid-gas mass transfer due to the moving microbubbles may also contribute to microbubbles shrinking along the serpentine microchannel.(142) We note, however, that these earlier demonstrations of passive dissolution of gas into the continuous liquid phase is a slow process, so relying on this passive mechanism alone is not sufficient to rapidly generate $O(1) \mu \mathrm{m}$ diameter microbubbles. (143)

The results in Fig. 4.2 also show that there is a monotonic decrease in final microbubble volumes, $V_{f} / V_{i}$, with increasing magnitude of the applied vacuum pressure, $P_{v}$. This evidence suggests that the applied vacuum pressure $P_{v}$, which is easily tunable, is a good control parameter for the resulting microbubble size. Importantly, we are able to shrink the microbubbles to diameters $\left(D_{f}=1-7 \mu \mathrm{m}\right)$ that are desirable to biomedical ultrasound and therapeutics applications.

In our approach, we create a negative pressure (i.e. vacuum) environment in the interdigitated vacuum microchannels that are adjacent to the main serpentine microchannel. Since we do not degas the continuous liquid phase before starting an experiment, the liquid is initially saturated with dissolved components of air at atmospheric pressure. However, PDMS is permeable to various components of air, namely gaseous oxygen, nitrogen, and carbon dioxide.(144) Therefore, we hypothesize that, in the 


\section{a Control}
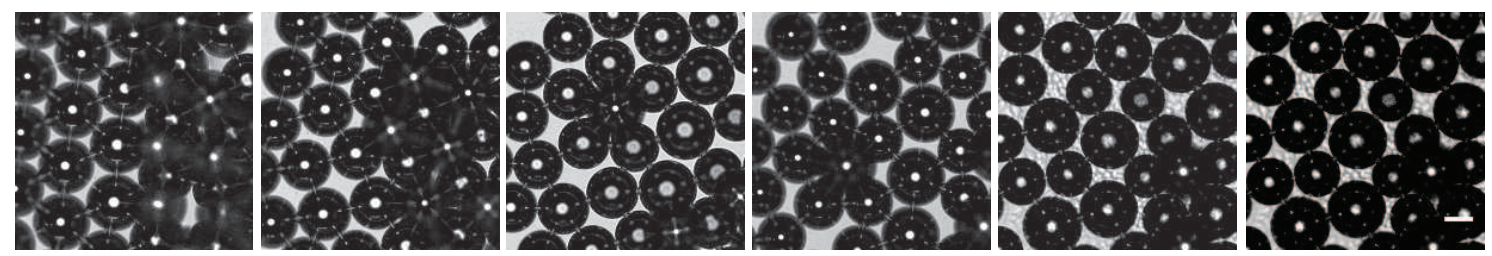

b $P_{v}=-70 k P a$
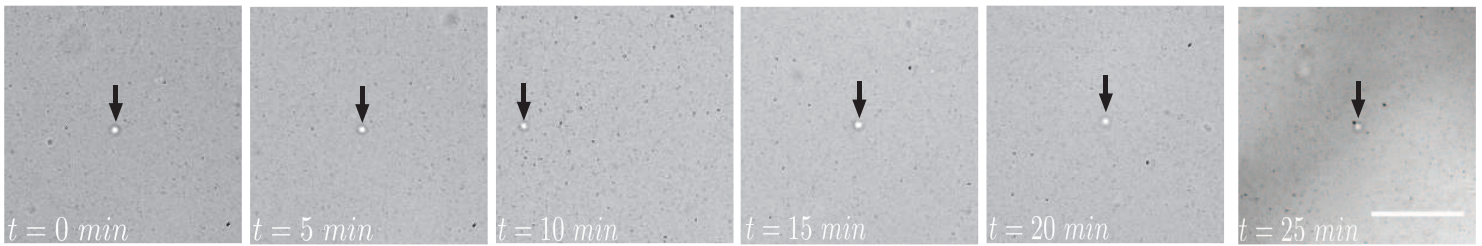

Figure 4.3: Representative sequential images of microbubbles collected at the outlet of the microfluidic device. (a) Microbubbles in the control experiment where the applied vacuum pressure, $P_{v}=0 \mathrm{kPa}$, observed via a 10x objective. (b) Microbubbles generated by a vacuum pressure, $P_{v}=-70 \mathrm{kPa}$, observed with a $63 \mathrm{x}$ objective. Here, arrows indicate the position of representative microbubbles in the sample. The microbubble shown in each frame may not be the same due to difficulties tracking the moving bubbles, but all microbubbles had diameter, $D_{f}=1-7 \mu \mathrm{m}$. Image frames are at 5 minute intervals for both (a) and (b). Scale bars represent $50 \mu \mathrm{m}$.

microfluidic device, the negative pressure in the vacuum microchannels cause gaseous components of air that are originally dissolved in the liquid continuous phase in the main serpentine channel, to permeate through the PDMS wall, $145 ; 144$ ) and exit via the vacuum microchannels. Simultaneously, the air inside the microbubbles dissolve into the liquid continuous phase as the bubble-liquid system attempts to continuously attain thermodynamic equilibrium-leading the microbubbles to shrink.

The resulting microbubbles after shrinkage are stable. The shrinking process removes molecules of air from the microbubbles, instead of, for example, compressing the microbubbles in a high pressure environment. Therefore, the microbubbles remain in a thermodynamic equilibrium state in the continuous liquid phase even after returning to an atmospheric pressure environment.

Figs. 4.3 and 4.3 show representative experimental images of the microbubbles collected at the outlet of the microfluidic device observed using 10x and 63x microscope objectives, respectively, at different points in time. In Fig. $4.3 \mathrm{a}$, the microbubbles are from the control experiment where the vacuum pressure, $P_{v}=0 \mathrm{kPa}$. Fig. $4.3 \mathrm{~b}$ shows microbubbles from an experiment where the vacuum pressure is set to $P_{v}=-70 \mathrm{kPa}$. We define the initial time $t=0 \mathrm{~min}$ as the moment when the sample is first collected from the outlet. Subsequent sequential images are taken at intervals of 5 min apart. For this particular experiment, the vacuum pressure of $P_{v}=-70 \mathrm{kPa}$ results in microbubbles with diameters, $D_{f}$, in the range of $1-7 \mu \mathrm{m}$. Both sets of images in Fig. 4.3 show that the collected microbubbles approximately maintain their size-stability even after $25 \mathrm{~min}$. 


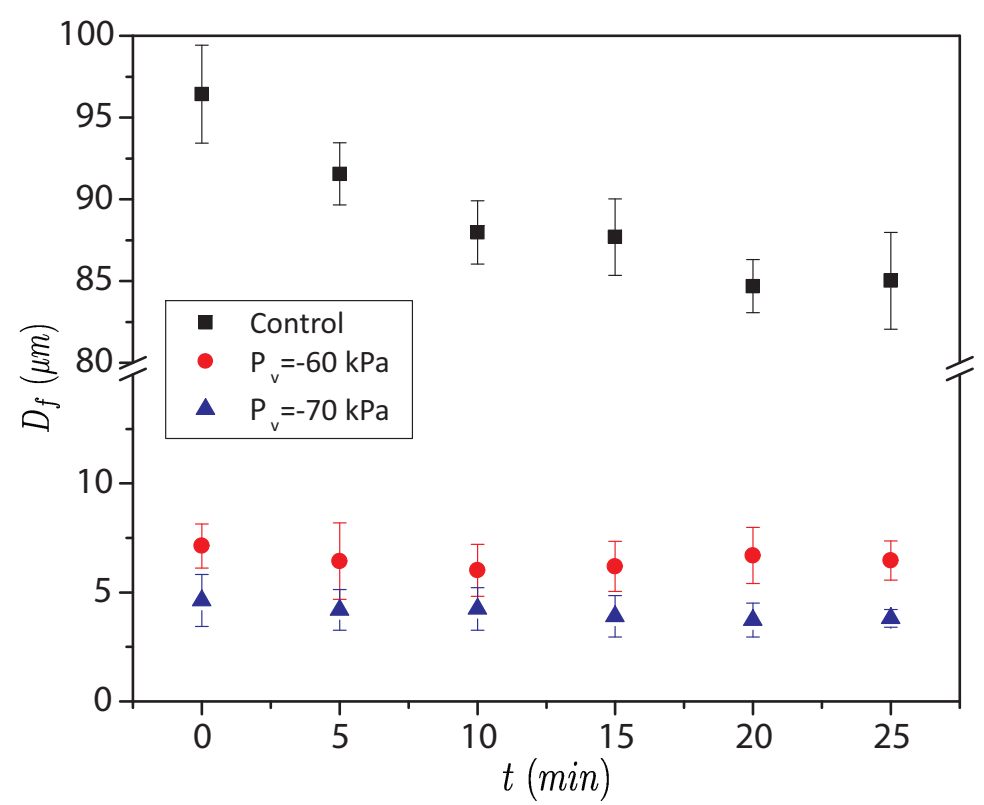

Figure 4.4: A plot of the diameter $D_{f}$ of the collected microbubbles versus time $t$. The data shown are from three representative experiments using vacuum pressures of $P_{v}=0,-60$, and $-70 \mathrm{kPa}$. Error bars represent one standard deviation for 10 samples.

Fig. 4.4 is a plot of the diameter, $D_{f}$, of collected microbubbles, versus time $t$. Here, we report data from three experiments where the applied vacuum pressure $P_{v}=0,-60$, and $-70 \mathrm{kPa}$. The plot shows that, for the control experiment, where $P_{v}=0 \mathrm{kPa}$, the diameter $D_{f}$ of the microbubbles decreases by approximately $10 \%$ over a period of 25 minutes. Microbubble diameters $D_{f}$ remain very stable for the experiments where the applied vacuum pressures $P_{v}=-60$ and $-70 \mathrm{kPa}$. Critically, this result is evidence that our microfluidic approach for vacuum-shrinking microbubbles is capable of making stable microbubbles that are in relevant length-scale of $1-7 \mu \mathrm{m}$ diameter.(118, 15)

\subsection{Conclusion}

For the first time, we demonstrate a microfluidic technique to reduce the size of microbubbles by applying vacuum pressure in interdigitated microchannels that are adjacent to the microchannels containing the flow of a bubble suspension. By tuning a single parameter, the applied vacuum pressure, our approach shrinks microbubbles to the $1-7 \mu \mathrm{m}$ diameter relevant length-scale that is desirable in biomedical ultrasound and therapeutic applications. Using this method, we show that microbubbles which are originally more than $100 \mu \mathrm{m}$ in diameter can be reduced to a few micrometers in diameter by controlling the applied vacuum pressure. We also show that the resulting microbubbles remain stable in atmospheric condition for at least 25 minutes. 



\section{Chapter 5}

\section{Conclusions and future work}

\subsection{Summary of findings}

The work in this thesis describes the first demonstration of an acoustic flow cytometer using simultaneous ultrasound backscatter and photoacoustics with ultra-high frequencies above $100 \mathrm{MHz}$. This section summarizes the significant findings from Chapters 2 through 4.

\subsubsection{Stable flow focusing using microfluidics (Chapter 2)}

Flow cytometry requires stable flow focusing to guide sample cells through a target region in a microchannel. To achieve a stable focused flow, we developed a simple microfluidic device that hydrodynamically focuses a sample flow. We use hydrostatic pressure, generated by liquid columns connected to the inlets, to drive sample and sheath flows. Variation in width of the focused flow due to hydrostatic driven flows is compared to the variation of the focused flow width generated by standard syringe pumps. The results show that the stability of hydrostatic pressure driven flow focusing is significantly better than the stability achieved by the syringe pumps. Furthermore, we found that there is an association between the width of the focused flow and the ratio of sample and sheath column heights. This approach of flow focusing is suitable for applications that are sensitive to the fluctuation of the flow and can have applications in various microfluidic flow cytometry applications.

The results from this chapter concluded that a pressure driven system generates a more stable hydro-

dynamic flow focusing compared to a syringe pump based system. Hence, in the following experiments we used pressure pumps to drive the microfluidic flows. 


\subsubsection{Acoustic based label-free flow cytometer development (Chapter 3)}

In this chapter, we demonstrate a novel label-free microfluidic flow cytometer that uses both ultrasound backscatter and photoacoustic signals to analyze individual micrometer-sized particles, and cells. An ultrasound transducer that has a center frequency of $375 \mathrm{MHz}$ and a 532 nm pulsed laser are integrated onto a microfluidic system that 3D flow focuses a sample flow through the focus of both ultrasound and laser beams. Each sample particle or cell passing through the focus is simultaneously interrogated by ultrasound and laser pulses to generate ultrasound backscatter and photoacoustic signals. These ultrasound backscatter and photoacoustic signals are strongly dependent on the size, morphology, mechanical properties, and absorption properties of the particles or cells. These properties can be extracted by analyzing the unique features in the power spectrum of the detected signals. This acousticbased technique allows us to rapidly analyze individual single cells of a large population for applications such as detecting circulating tumor cells detection and characterizing various healthy and diseased cells. This is the first demonstration of the flow cytometry system using ultrasound and photoacoustics to analyze individual cells.

\subsubsection{Microfluidic microbubble shrinking system (Chapter 4)}

The microfluidic bubble shrinking system demonstrated in this chapter is able to generate microbubbles of diameters more than $100 \mu \mathrm{m}$ and shrink them to the desired size using a single control parameter, vacuum pressure. A PDMS based microfluidic device consists of interdigitated vacuum microchannels adjacent to the microchannel containing the flow of microbubbles. When vacuum is applied to these interdigitated channels, air dissolved in the continuous phase liquid is removed through the PDMS wall. To maintain the equilibrium, air diffuses from the flowing microbubbles, as a result, the bubbles gradually shrink as they drift downstream. Using this approach, we are able to generate microbubbles of diameter 1-7 $\mu \mathrm{m}$ that can be used for ultrasonic biomedical applications such as imaging and drug delivery. We show that these shrunken bubbles are stable in an ambient environment for at least 25 minutes. We claim that our microbubbles have comparable stability as commercial microbubbles because these bubbles are made of similar lipid composition. We anticipate that, scaling-up the system, we will be able to produce enough microbubbles of the desired size for diagnostic ultrasound imaging and therapeutic applications.

\subsection{Contribution}

The research presented in this thesis demonstrates the successful development of a novel label-free acoustic flow cytometer that uses simultaneous ultra-high frequency ultrasound, above $100 \mathrm{MHz}$, and photoacoustics, to analyze individual micron sized objects. To acomplish the goal of developing this acoustic flow cytometer, the following amis were fulfilled: (i) develop a needle-based microfluidic device to hydrodynamically 3D flow focus a sample flow in a microchannel. Using this device, we show that the hydrostatic pressure driven focused flow is significantly more stable than the focused flow gener- 
ated by the syringe pumps that are commonly used for microfluidic applications. (ii) Use the acoustic flow cytometer to detect and differentiate individual cell in a heterogeneous solution. We differentiated the label-free the red blood cells and white blood cells in a solution using the acoustic flow cytometer. The results from the label-free experimentation agree with the results from the fluorescent activated cell sorting system, which uses the fluorescent molecules, present bonded to the white blood cells, to differentiate them. (iii) Develop a simple microfluidic device that generates microbubbles larger than $100 \mu \mathrm{m}$ in diameter and shrinks them to a desired size. Microbubbles generated from this microfluidic device can be used for ultrasonic diagnostic and therapeutic applications.

Lastly, this research provides a practical step towards the commercial development of acoustic flow cytometry that is capable of detecting solid, liquid, and gaseous micrometer and sub-micrometer objects. This technique can be clinically relevant for single cell analysis, circulating tumor cell detection, and various diagnostic applications.

\subsection{Future work}

A novel ultrasound and photoacoustic-based microfluidic flow cytometer has been developed. The results are presented as proofs of principle and show that the results from the system are comparable to the results from the existing gold standard technique for differentiating between RBCs and WBCs. My interest in future research is to use the system for various biomedical applications that are not possible in the existing commercial flow cytometry systems. Here, I will highlight some of the ongoing research using the acoustic flow cytometer, which were initiated during the dissertation period.

\section{Nucleus-to-cytoplasmic (N:C) ratio calculation of single cells}

The nucleus-to-cytoplasmic $(\mathrm{N}: \mathrm{C})$ ratio is the ratio of cross-sectional area of the nucleus to the cytoplasmic area of a cell, where the cytoplasmic area is the difference between the cross-sectional area of the cell and its nucleus. In general, tumor cells have larger nucleus compared to that of normal cells. Hence, the N:C ratio is used to assesses the presence of malignant disease while assessing histology.(146)

The goal of this ongoing project is to calculate the $\mathrm{N}: \mathrm{C}$ ratio of label-free cells using acoustic flow cytometer to detect circulating tumor cells in blood. A circulating tumor cell has enhanced N:C ratio compared to other blood cells and hence the tumor cell can be distinguished. A cell nucleus is a good absorber of UV light. Modifying the acoustic flow cytometer with a pulsed UV laser, it is possible to detect photoacoustic signals from the nucleus of individual cells. Using both ultrasound backscatter signal from a single cell, and the photoacoustic signal from the nucleus of the same cell, it is possible to estimate the size of the cell and its nucleus. The size of the cell and the nucleus can be then used to calculate the N:C ratio of a single cell and eventually differentiate a circulating tumor cell from other blood cells. 
Preliminary results from the signals detected by the acoustic flow cytometer from nucleus stained MCF7 breast cancer cells using $532 \mathrm{~nm}$ pulsed laser are presented in Appendix D.

\section{Characterization of microbubbles and nanobubbles}

Microbubbles of 1-7 $\mu \mathrm{m}$ in diameter are used as intravascular contrast agents for ultrasonic imaging applications, drug delivery vehicles, and therapeutics.(147) To overcome the size barrier of the microbubbles for the abovementioned applications, nanobubbles are used. For both micro and nanobubble applications, it is important to have characteristic information of individual bubbles and their response to an ultrasound pulse. However, there is no robust and rapid commercial technique that detects and analyzes the ultrasonic response of these micro and nanobubbles. Hence, the goal of this ongoing project is to develop a technique, using acoustic flow cytometer, to detect individual micro and nanobubbles and analyze their ultrasonic response to size and characterize them.

The microfluidic device developed in the Chapter 4 generates microbubbles of specific size by controlling the vacuum pressure in the system. These microbubbles of known size are then flown through the acoustic flow cytometer to obtain the ultrasound backscatter signals from the individual microbubbles. Likewise, when flowing nanobubbles through the acoustic flow cytometer, we are able to show that the acoustic flow system is capable of detecting ultrasound backscatter from individual nanobubbles. This ongoing project targets to show proofs of the principle that the individual micro and nanobubbles are detected by the acoustic flow cytometer. Furthermore, we would like to develop algorithms to process detected ultrasound backscatter signals to characterize the individual micro and nanobubbles. Similarly, we hypothesize that a nanobubble tagged with optical absorber molecule produces significant photoacoustic signal that can be detected by the transducer in the acoustic flow cytometer. Such photoacoustic signal would provide information on the shell properties of the individual nanobubbles.

The preliminary results of the detection of ultrasound backscatter and photoacoustic signals from individual micro and nanobubbles using the acoustic flow cytometer are presented in Appendix D. 


\section{Appendix A}

\section{Supplementary information for chapter 2}

This section is published as the supplementary information for the following paper

Gnyawali, V., Saremi, M., Kolios, M. C., and Tsai, S. S. H. (2017). Stable microfluidic flow focusing using hydrostatics. Biomicrofluidics, 11(3), 34104. https://doi.org/10.1063/1.4983147

\section{Controlled focused flow diameter hydrostatic experiment}

In the hydrostatic flow focusing experiments, the flow rate of liquids flowing in the microchannels depends on the hydrostatic pressure from liquid columns. The hydrostatic pressure is expressed by the relation,

$$
\nabla P=\rho g H
$$

where $H$ is the height of the liquid column, $\rho$ is the density of the liquid, $g$ is the acceleration due to gravity. In hydrostatic pressure driven flow focusing, the sample and sheath flows are actuated by the hydrostatic pressure from the individual liquid columns. The faster flowing sheath flow clads the slower sample flow. Therefore, during an experiment, the height of the sheath liquid column, $H_{2}$, decreases faster than the height of the sample liquid column, $H_{1}$.

The focused flow diameter, $d_{s}$, downstream of flow focusing junction, is related to the ratio of the liquid column heights as shown in Eq. A.1. Due to the more rapid decrease of the sheath flow liquid column height, $H_{2}$, the sheath flow speed decreases relative to the sample flow speed, and the focused flow diameter, $d_{s}$, increases as a result. An experiment can continue as long as the expanding focused

flow diameter, $d_{s}$, is still within the acceptable range for specific applications or until any of the liquid columns empties. The total experimental time can be increased either by using wider liquid column 
containers with larger diameter so that the liquid column heights decrease slower or by continually adding liquid to the liquid columns. In our experiments, we take cylindrical liquid columns of internal diameters of $3 \mathrm{~mm}$ and $8 \mathrm{~mm}$ for sample and sheath liquids, respectively. The columns are filled up to a specific height with ink, as a sample fluid, and deionized (DI) water, as a sheath fluid. In the microchannels, ink is hydrodynamically flow focused by DI water. Using a high-speed camera, we take videos of the focused flow at different time intervals. The videos are analyzed using the method described in the manuscript to calculate the average diameter, $d_{s}$.

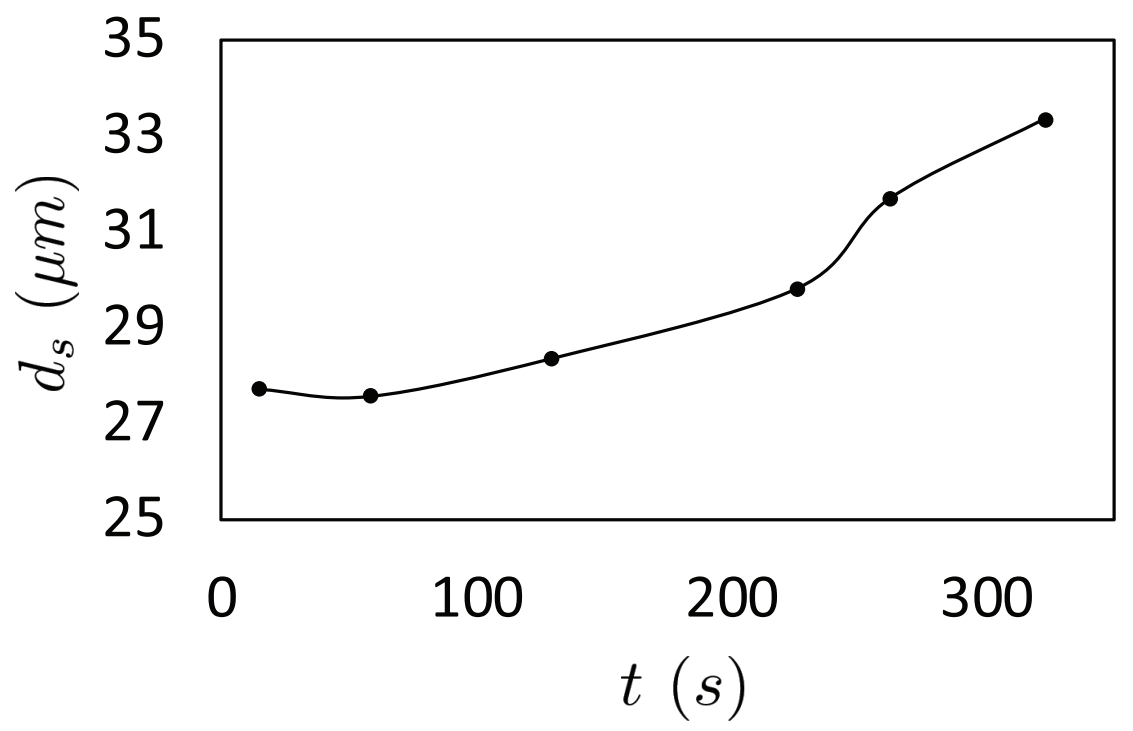

Figure A.1: A plot of an average focused flow diameter, $d_{s}$, versus total experimental time, $t$, for an experiment using hydrostatics over a period of $5 \mathrm{~min}$.

Fig. A.1 is a plot of experimental results that show the average focused flow diameter, $d_{s}$, versus instantaneous experimental time, $t$. In this specific experiment, the first measurement is taken $15 \mathrm{~s}$ after the stable focused flow is generated. In the course of time, the height of the liquid columns decreases, eventually increasing the focused flow diameter, $d_{s}$. The plot shows that the diameter, $d_{s}$, of the focused flow changes from $28 \mu \mathrm{m}$ to $33 \mu \mathrm{m}$ in a period of $300 \mathrm{~s}$ (approximately $5 \mathrm{mins}$ ), and increases from 28 $\mu \mathrm{m}$ to $29 \mu \mathrm{m}$ in the first $200 \mathrm{~s}$ (approximately $3.3 \mathrm{mins}$ ). 


\section{Appendix B}

\section{Supplementary information for chapter 3}

This section is supplementary information submitted with the following paper

Gnyawali, V., Strohm, E. M., Wang, O., Tsai, S. S. H., Kolios M. C. (2018) Microfluidic acoustic flow cytometry: a label-free approach, Scientific Reports.(submitted)

Slow and fast-time signals
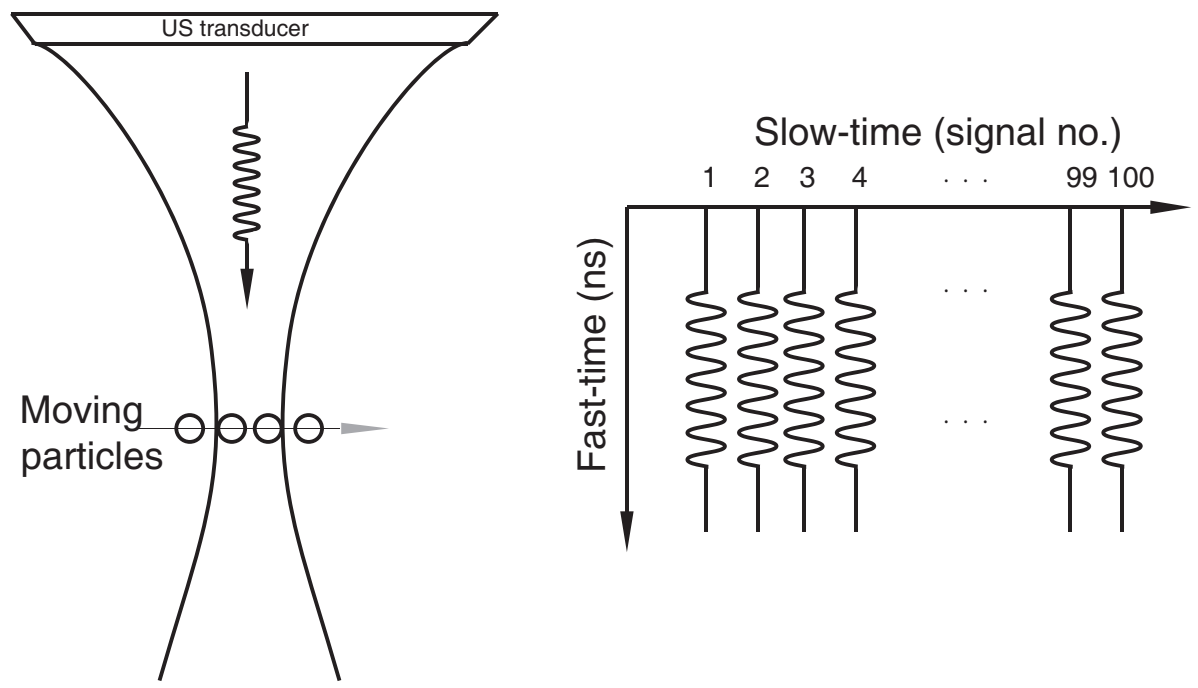

Figure B.1: The schematic illustration of slow and fast-time axes representing signals detected by the US transducer while a particle flows through the focal zone of the transducer. 


\section{Autofluorescence detected from polystyrene particles using FACS}
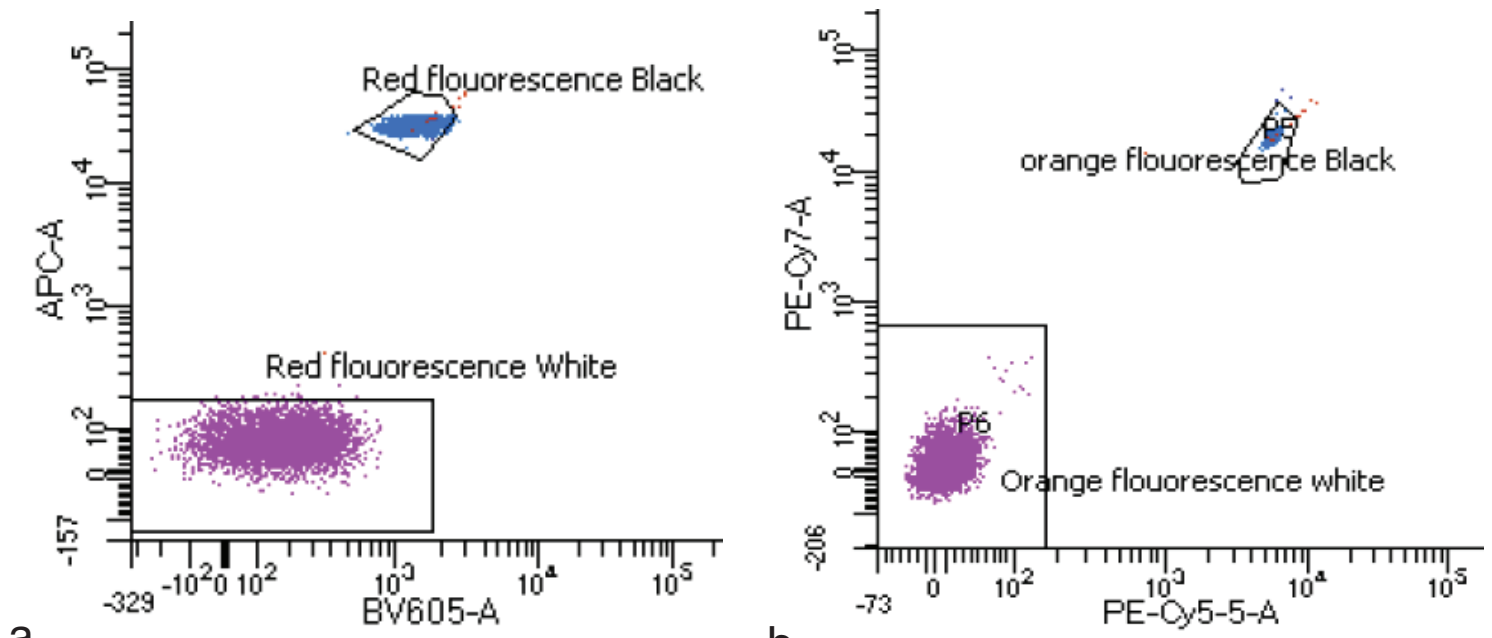

Figure B.2: FACS results from $3 \mu \mathrm{m}$ diameter polystyrene black and white particles, which are denoted as black and white, respectively. Data detected in the APC and BV605 channels (a) and PE-Cy7 and PE Cy5 channels (b) for the same sample of particles.

The sample contained a mixture of black and white polystyrene particles of $3 \mathrm{~m}$ diameter. The autofluorescence signals produced by white and black particles at different channels, namely APC, BV605, $\mathrm{Cy} 5$, and $\mathrm{Cy} 7$, are combined to distinctly separate black particles from white particles using FACS system. This allowed the comparison with the results from the acoustic flow cytometry.

\section{Microfluidic device fabrication}



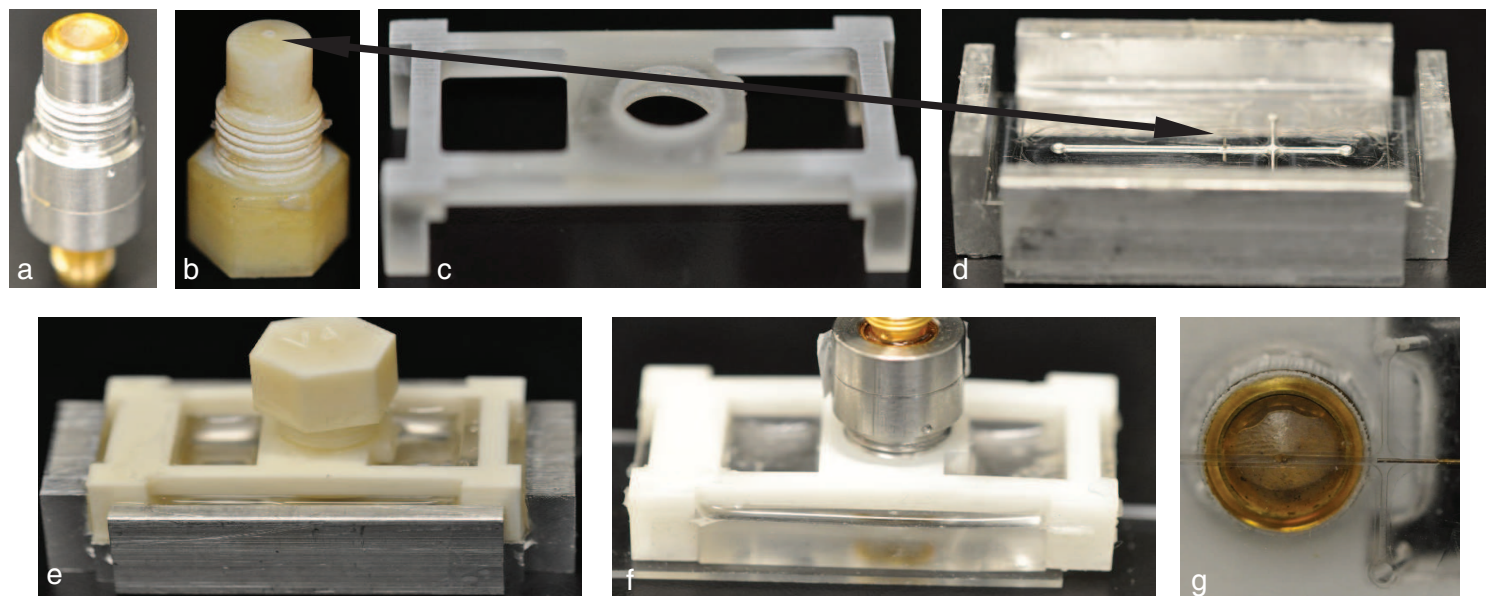

Figure B.3: Components and steps used to fabricate the microfluidic device. a) The US transducer; b) the 3D printed transducer mold; c) the 3D printed alignment frame; d) the CNC machined metal mold. (e) The transducer mold replaced by the US transducer in the final microfluidic device. f) The backside view of the device.

\section{Flow diagram of the flow system}

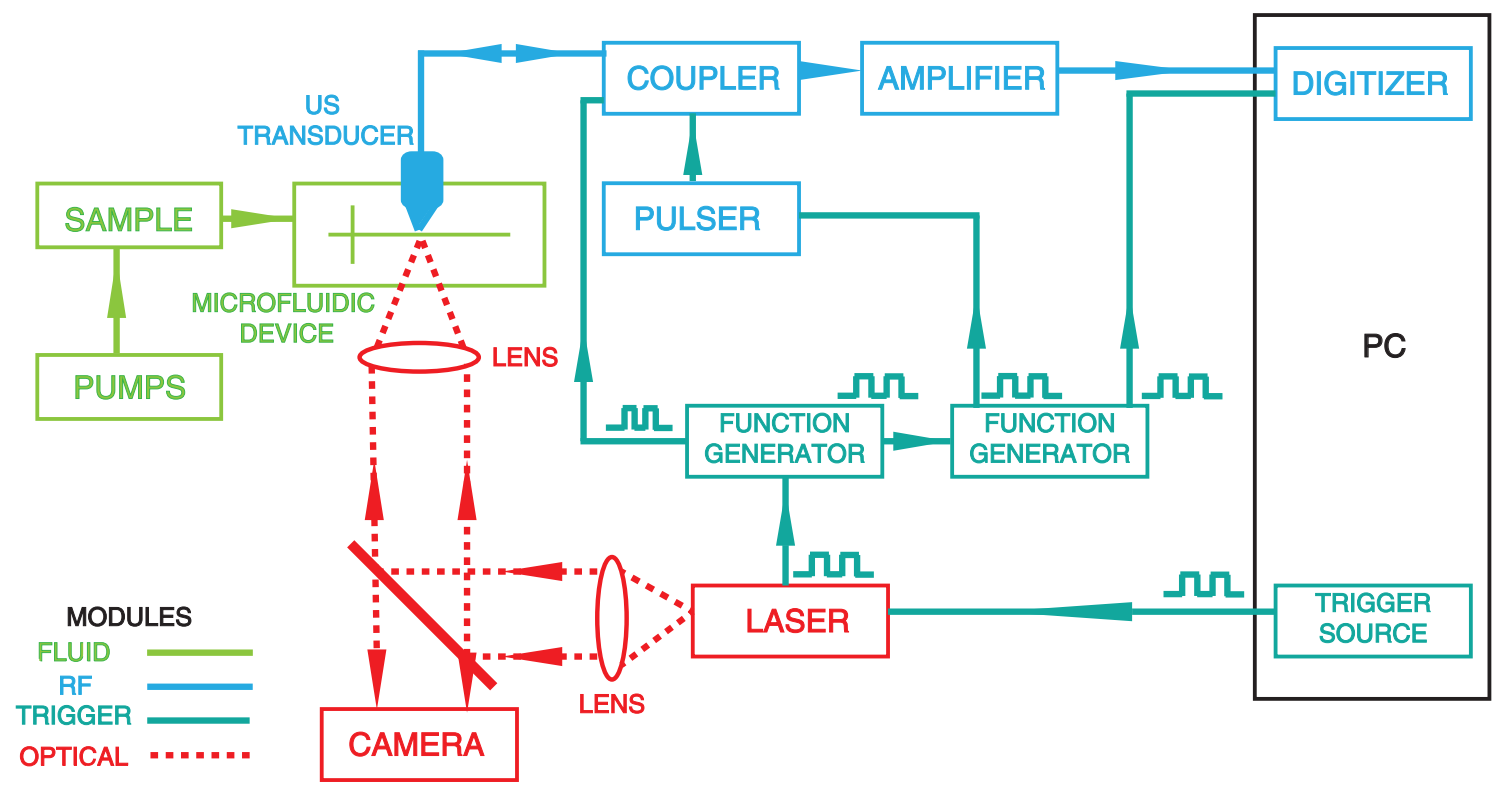

Figure B.4: Flow diagram of the acoustic flow cytometer. Arrows indicate direction of travel of either signals, fluids, or light depending on the module.

The acoustic flow cytometer contains four distinct modules, namely fluid, RF, trigger, and optical 
(Fig. B.4. The fluid module consists of the microfluidic device, tubing, and pumps. The hydrodynamically focused sample flow passes through an interrogation zone inside a microfluidic channel. The individual cells or particles in the sample flow are individually interrogated by both ultrasound and laser pulses, simultaneously.

The RF module accounts for all the components that are used for emitting ultrasound and detecting, and recording both ultrasound and photoacoustic signals. The tools that generate, synchronize, and control the triggers are grouped as a trigger module. The microfluidic device together with the pumps are grouped as a fluid module. Finally, all the optical systems, including lasers, optical components, and camera, are in the optical module.

A ultrasound transducer is integrated with a microfluidic device. This transducer switches between emission and receiving modes. During the emission mode, a ultrasound pulse is emitted towards the sample particles and during the receiving mode, it detects the ultrasound signals from the particles. The modes are controlled by a trigger-controlled coupler, which switches the connection of the transducer with a pulse generator and an amplifier for emission and receiving modes, respectively. The detected signals from the transducer are amplified by an amplifier and are recorded by a digitizer for post-processing. The complete process is shown as RF module in the flowchart of Fig. B.4.

The synchronization of the components in the system is maintained by various trigger signals. A control trigger operating at $4 \mathrm{KHz}$ is generated by a PCI card and triggers a pulsed laser source. Each laser pulse generates a synced TTL signal to activate the pulse generator, digitizer, and the coupler to switch the transducer modes. This complete process flow is shown as Trigger module in the Fig. B.4.

Simultaneously, laser pulses are also focused on the particles by a 10x optical objective. The laser pulses generated from a laser source transmits through an optical fiber and multiple reflecting and dichroic mirrors before being focused by the objective. A camera is connected to observe the microfluidic channel in real-time. 


\section{Appendix $\mathrm{C}$}

\section{Supplementary information for chapter 4}

This section is published as the supplementary information for the following paper

Gnyawali, V.*, Moon, B.-U.*, Kieda, J., Karshafian, R., Kolios, M. C., and Tsai, S. S. H. (2017).

Honey, I shrunk the bubbles: microfluidic vacum shrinkage of lipid-stabilized microbubbles. Soft Matter, 13 (22), 4011-4016. https://doi.org/10.1039/C7SM00128B

Flow diagram of the experimental setup 


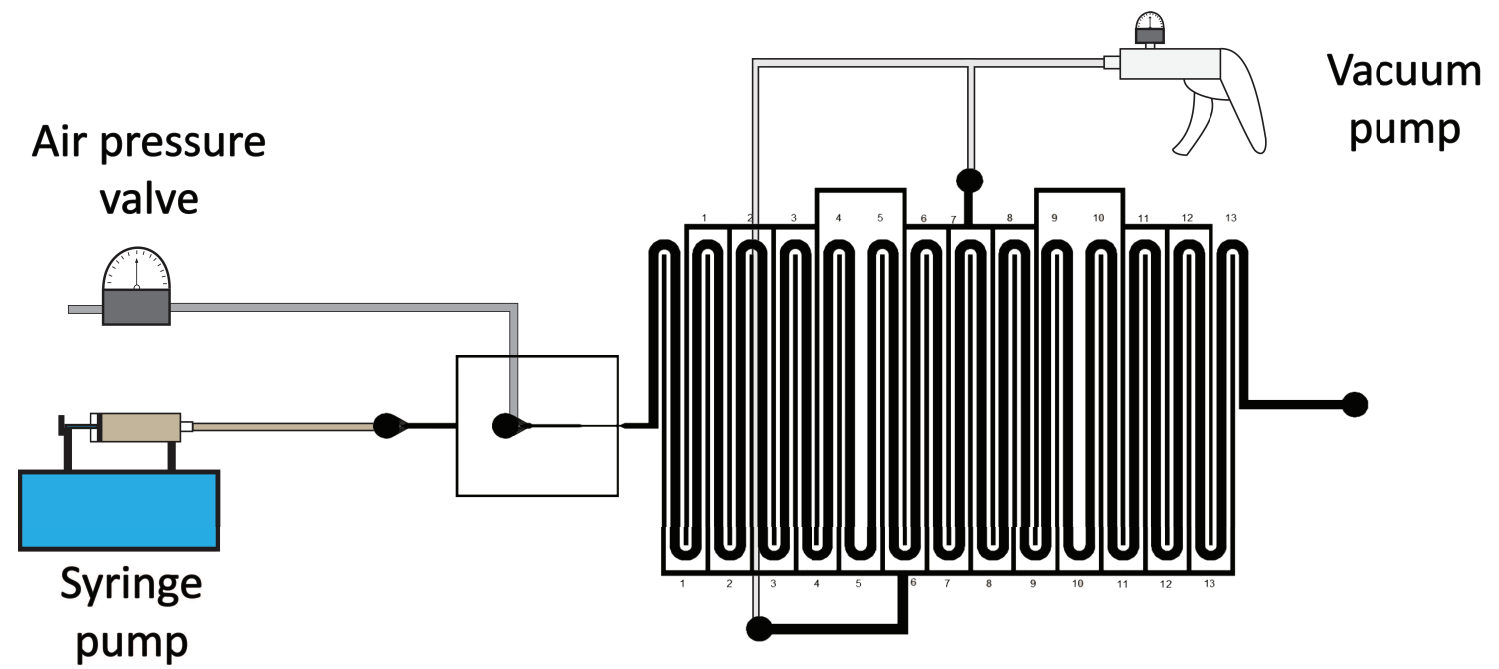

Figure C.1: The schematic diagram of the microbubble shrinkage system. We supply air through a pressure valve where we fix the inlet air pressure to $P_{a}=4$ psi for all the experiments. Simultaneously, we infuse the lipid-mixture using a syringe pump. The infuse flow rate is also fixed at $\mathrm{Q}=4 \mu \mathrm{L} / \mathrm{min}$ for all the experiments. Our only tuned variable, vacuum pressure, $P_{v}$, is supplied through two inlets using a Mityvac vacuum pump, which consists of an integrated control valve. The bubble is generated at the flow focusing orifice between air and lipid mixture channels, and flows downstream through the serpentine channel where the microbubbles shrink due to the effect of vacuum in the adjacent interdigitated vacuum channels. The schematic diagram is not in scale.

Calculation of the volume of discoid and spherical microbubbles

a

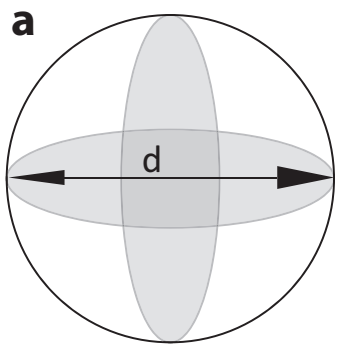

b

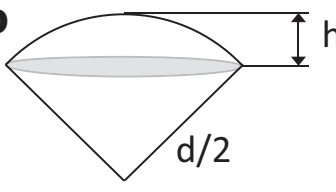

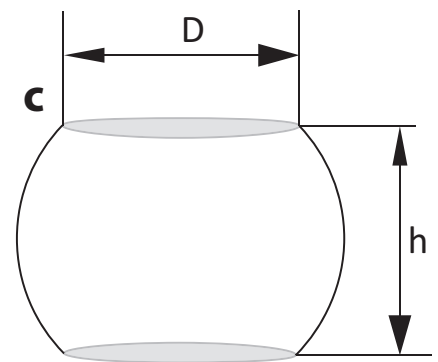

Figure C.2: Schematic representation of different shapes of microbubbles. (a) Spherical microbubble, (b) spherical cap of a microbubble, and (c) a discoid microbubble.

We use the following equations to calculate the volume of the discoid microbubbles at the orifice and the spherical microbubbles at the downstream of the microchannel. In the simple experimental condition 
where the microbubble diameter $d$, is smaller than the channel height $h$, the microbubbles take on a spherical shape whose volume $V$ is given by (Fig. C.2 ),

$$
V=\frac{\pi d^{3}}{6}
$$

The volume $V$ of an equivalent spherical cap (Fig. C.2 ) is given by,

$$
V=\frac{\pi h_{c}{ }^{3}\left(3 R-h_{c}\right)}{3}
$$

where, the height of the cap is given by $h_{c}$.

In experimental conditions where the projected microbubble diameter, $D$, measured as the microbubble diameter from the top or bottom view using a microscope objective, is greater than the channel height $\mathrm{h}$, the microbubble is confined by the microchannel and takes on a discoid shape (Fig. C.2.). In this case, the volume $V_{d}$ of the discoid microbubble is given by reference (148),

$$
V=\frac{\pi\left(2 D^{3}-(D-h)^{2}(2 D+h)\right.}{3},
$$

where, the height $h$ of the channel is also the height of the discoid microbubble sectioned from the top and the bottom, and is defined as,

$$
h=2\left(0.5 d-h_{c}\right)
$$





\section{Appendix D}

\section{Future work}

\section{D.1 Measuring nucleus-to-cytoplasmic (N:C) ratio of single cells in a population}

The nucleus-to-cytoplasmic (N:C) ratio is an important parameter that is used to assess the presence of malignant disease while assessing histology.(146) Pathologists use post-processing techniques on optical microscopic images to measure the N:C ratio of individual cells.(146) However, in recent years image analysis has been automated by using software. Yet, the quality of the image taken and the automation process is limited by the users expertise in tissue preparation and the stereological techniques used to interpret the data taken from the histological results. 149 , 150) There are 3D imaging techniques, such as confocal microscopy, Cell-CT, that can be used to calculate the N:C ratio.(151) Nevertheless, these 3D imaging techniques lack the throughput for examining a large population of cells.(106) The acoustic flow cytometry technique, that was developed during the dissertation period, can serve as a promising alternative to existing techniques to calculate the N:C ratios at approximately 150 cells per minute.

The current version of the acoustic flow cytometer integrates a $375 \mathrm{MHz}$ ultrasound transducer and a $532 \mathrm{~nm}$ wavelength pulsed laser source. At this high frequency, ultrasound backscatter and photoacoustic signals can be used to estimate the cell and its nucleus size, respectively. The nucleus does not absorb significant optical energy at $532 \mathrm{~nm}$ wavelength and cannot generate detectable photoacoustic waves using the existing setup. However, to establish a proof of principle, cells with nuclei stained with a fluorinated dye, DRAQ5 fluorescent probe (Thermo Scientific, Waltham, MA, USA) were used to produce photoacoustic signals at $532 \mathrm{~nm}$. As a result, the flow system could be used to detect both the ultrasound backscatter and the photoacoustic signals generated by a cell and its nucleus, simultaneously.

The ultrasound backscatter and photoacoustic signals from an individual cell contain information about the overall size of the cell and its nucleus. $43,79,44,146)$ The signals are time-gated from the detected RF line, subjected to a Tukey window, and filtered using a 200 - $500 \mathrm{MHz}$ bandpass filter. Then 
the power spectra of the resulting signals are calculated using the Fast Fourier Transform. Similarly, the Faran scattering model and the Diebold model are used to generate theoretical spectra from liquid spheres of a diameter ranging from $3 \mu \mathrm{m}$ to $30 \mu \mathrm{m}$ for ultrasound backscatter and photoacoustics, respectively.(79, 106) An individual measured power spectrum is compared with the theoretical spectra based on the distance between measured spectral minima. This same technique of determining the size of a cell is used in the paper attached in the Appendix E. Using the cell and its nucleus diameters, the $\mathrm{N}$ :C ratio is calculated as the ratio of the cross-sectional area of its nucleus to the difference of the cross-sectional area, between the cell and its nucleus.

$$
N: C=\frac{A_{n}}{A_{c}-A_{n}},
$$

where $A_{n}$ is the cross-sectional area of a nucleus and $A_{c}$ is the cross-sectional area of a cell.
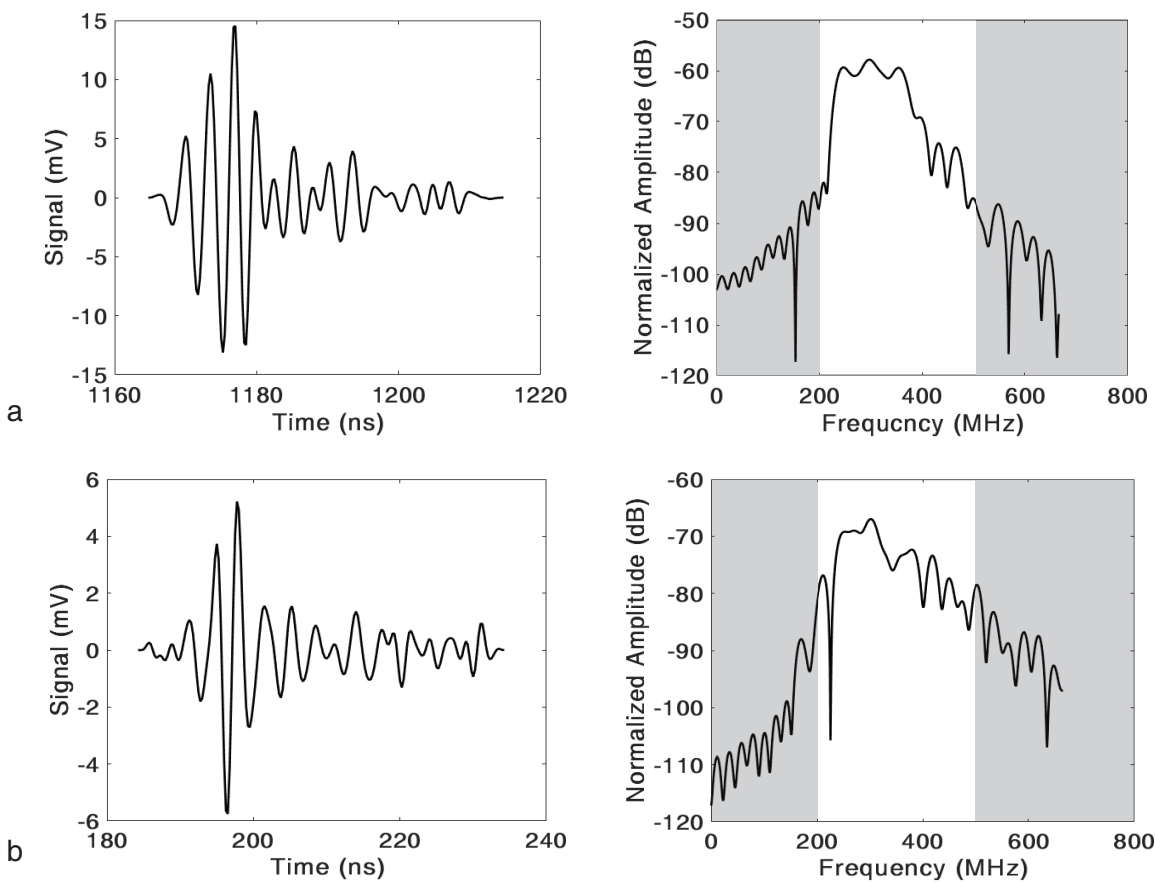

Figure D.1: a) A representative time-gated time domain ultrasound backscatter signal and frequency domain ultrasound backscatter and (b) photoacoustic signal detected from an MCF7 cell. The portion of the signal outside the bandwidth of the transducer is shaded grey in the figure.

Preliminary results from the experiments for detecting simultaneous ultrasound and photoacoustic signals from nuclei stained MCF7 breast cancer cells and the calculated size of the cells and their nuclei have shown promising results. In these experiments, both ultrasound and photoacoustic signals from DRAQ5 stained cells were simultaneously detected by the acoustic flow system. Representative time-gated ultrasound backscatter and photoacoustic signals from an MCF7 cell with their respective 

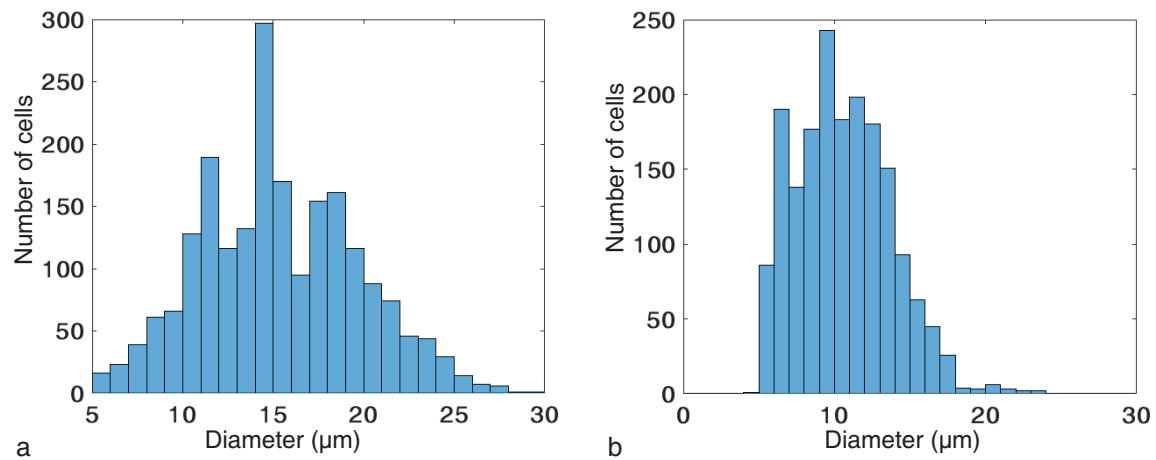

Figure D.2: Histograms showing a distribution of the estimated size of (a) cells and (b) nuclei. The size of the cells and their nuclei are estimated using the ultrasound backscatter and the photoacoustic signals detected from the acoustic flow cytometer, respectively.

frequency spectrum are shown in Figs. D.1 and b. Fig. D.2 shows histograms of the measured size distribution of the cells and their nuclei, respectively. From the data presented in Fig. D.2, the average size of the MCF7 cells was calculated as $16.3 \pm 4.8 \mu \mathrm{m}$ and the nuclei diameter were calculated to be $10.6 \pm 3.1 \mu \mathrm{m}$. To compare the results, the same batch of the MCF7 cells were sized using the Coulter counter (Beckman Coulter Ltd., High Wycombe, U.K.), a standard technique to size single cells. The Coulter counter measured their average size to be $18.2 \pm 3.9 \mu \mathrm{m}$ as the average size of the MCF7 cells, with the measured sizes in the range of $10-25 \mu \mathrm{m}$. Using the estimated sizes of the cells and nuclei from the acoustic flow cytometer, the average $\mathrm{N}: \mathrm{C}$ ratio of the MCF7 cells was calculated as $0.9 \pm 0.9$. This value does not correspond well with the literature value, $1.9 \pm 1.0 .(106)$ However, the result presented here is from thousands of cells, whereas the value presented in the literature is from only nine cells. Future work will focus on resolving this discrepancy.

The sizing technique, presented above, gives a reliable result when the frequency spectrum of a measured signal contains at least two minima that are within the bandwidth $(200-500 \mathrm{MHz})$ of the transducer. However, not all signals from cells or nuclei contain at least two minima. Specially, for photoacoustics the absorbers with diameter smaller than $7 \mu \mathrm{m}$ do not have two minima within the bandwidth. Beside spectral peaks, the signal to noise ratio of the detected signal also plays a significant role in estimating the size for cells and their nuclei. For example, this algorithm sized the AML cells (average diameter $10.2 \pm 3.6 \mu \mathrm{m}$ ) consistently, whereas their nuclei size was inconsistent. This inconsistency is attributed to the fact that the cells, unlike nuclei, are larger than 7-8 $\mu \mathrm{m}$ (results not shown here). In future, I would like to optimize the existing sizing algorithm to better estimate the size of cells and nuclei of different cells.

The goal of future research would be to detect circulating tumor cells through label-free signal measurements in order to calculate the $\mathrm{N}: \mathrm{C}$ ratio in whole blood. As the nucleic acids present in the 
nucleus are good absorbers of UV light, I eventually aim to integrate a pulsed UV laser in the acoustic flow cytometry system, which will enable generation of photoacoustic signals from the nucleus of labelfree cells. The system will be able to produce both ultrasound backscatter and photoacoustic signals from individual label-free cells, simultaneously.

\section{D.2 Characterization of microbubbles and nanobubbles}

Microbubble suspensions used for ultrasonic applications generally contain bubbles measuring 1-7 $\mu \mathrm{m}$ in diameter. These microbubbles have been used for applications beyond that of imaging contrast agents, such as drug delivery vehicles, super-resolution imaging, and therapeutics.(147) This growth in the use of bubbles has increased the importance of better understanding the behaviour of microbubbles and to improve microbubble characterization methods.(147) Existing commercially available technologies, such as the Coulter counter or Multisizer, the Mastersizer (Malvern Instruments, Worcestershire, U.K.), and the Archimedes ${ }^{\circledR}$ (resonant mass measurement based system, Malvern Instruments, Malvern, U.K.) are used for sizing and counting microbubbles. Similarly, various methods, such as optical microscopy, laser diffraction, and single-particle optical light scattering are commonly used for sizing and counting microbubbles in suspension. $(152,153)$ Even though these existing techniques and methods provide good results of size and concentration of microbubbles in sample solutions, they are impractical due to their high cost of implementation, and limited throughput.(147, 154)

Microbubbles are intravascular contrast agents as they remain within the vasculature due to their size. This size-dependent limitation of the microbubbles can be overcome by nanobubbles. Recent research has shown that the nanobubbles can be transported through intercellular gaps in diseased tissues to reach the target cells and tissues outside the capillaries. (155) Hence, there is great potential for nanobubbles in applications, such as contrast-enhanced diagnostic imaging, blood-brain barrier disruption, and transport of drugs for cancer therapy.(156, 157) Furthermore, these nanobubbles are advantageous in the tumor microenvironment where vasculature exhibits the enhanced permeability and retention (EPR) effects for molecules and nanoparticles due to the presence of pores ranging from $380-780 \mathrm{~nm} 157$. 158: 159) It is very important to understand properties of the individual nanobubbles before using them for such biomedical applications. However, there is no commercial technique that can analyze single nanobubbles in a suspension. A recently available commercial instrument, Archimedes ${ }^{\circledR}$, uses resonant mass measurement to measure the size of nanobubbles. (20) The Archimedes ${ }^{\circledR}$ is only useful for bubble samples with a small size distribution. For example, the Archimedes ${ }^{\circledR}$ is not able to detect microbubbles (diameters above $2 \mu \mathrm{m}$ ) in a nanobubble solution or smaller nanobubbles (diameters of few hundred nanometers) in a microbubble solution. In most cases, both microbubbles and nanobubbles co-exist in

a solution, which increases the uncertainty of the results obtained from the Archimedes ${ }^{\circledR}$. Besides the measurement uncertainty, samples used in Archimedes ${ }^{\circledR}$ require a high concentration of bubbles.

Microbubbles generated from the microfluidic system presented in chapter 4 can be used for mi- 
crobubble characterization using the acoustic flow cytometer. The microbubble generation system has a good control over the size of the resulting bubbles and the measurement system can be integrated downstream for the channel that bubbles are made in. Results from chapter 4 show that the bubbles are in the range of a few micrometers at vacuum pressure below $-70 \mathrm{kPa}$. I hypothesize that by increasing vacuum pressure or increasing interaction time between the microbubbles and the vacuum, it is possible to further shrink the microbubbles to nanobubbles. Even though it is beyond the scope of this thesis, I hypothesize that the microbubbles shrink further to nanobubbles.

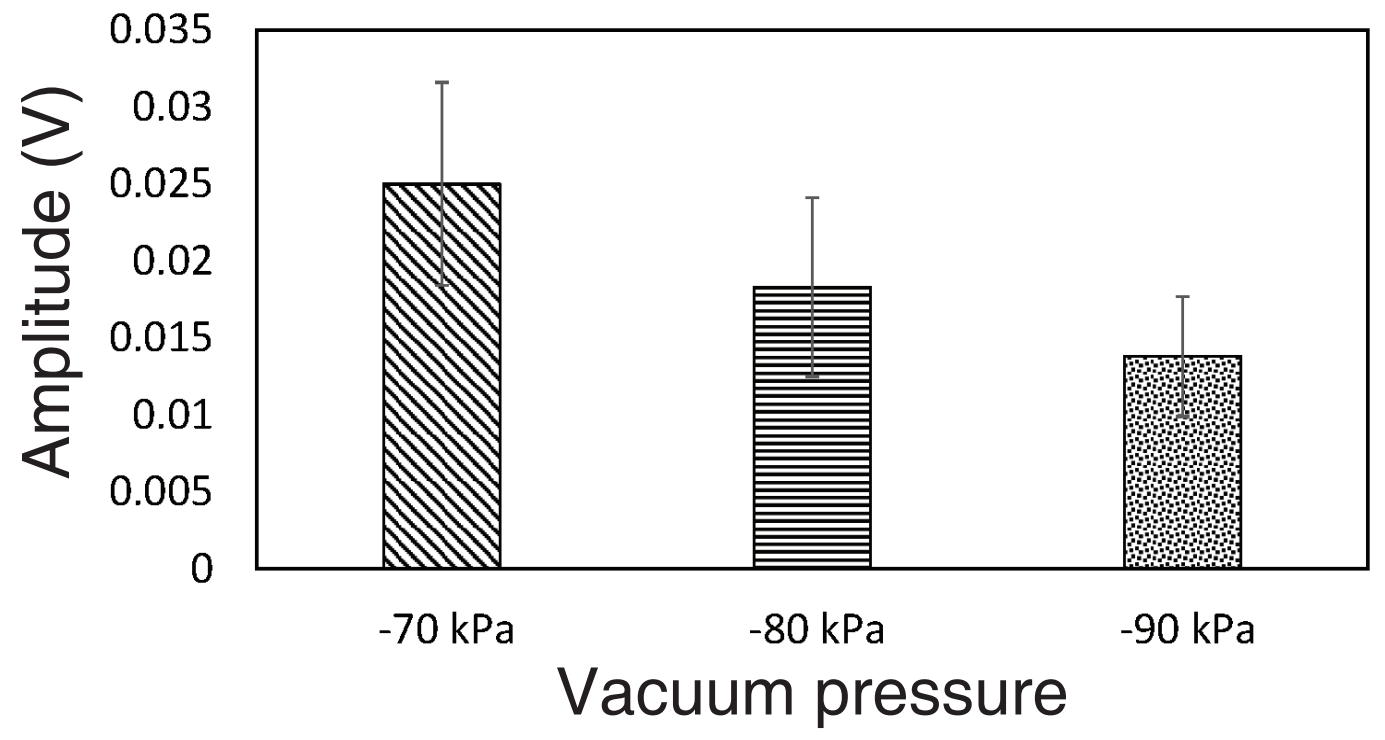

Figure D.3: The average amplitude of the ultrasound backscatter signals detected from the individual shrunken micro/nanobubbles at different vacuum pressures. Error bars represent one standard deviation from at least 20 microbubbles for each vacuum pressure.

As a proof of principle experiment to detect individual microbubbles using the flow system, the shrunken microbubbles are used. Fig. D.3 shows the average amplitude of the ultrasound backscatter signals detected from individual micro/nanobubbles. These bubbles were generated by the shrinking process using vacuum pressures, $V_{p}=-70 \mathrm{kPa},-80 \mathrm{kPa}$, and $-90 \mathrm{kPa}$. The graph shows that the average amplitude of the ultrasound backscatter decreases with an increase in vacuum pressure. The amplitude of the backscatter signal is strongly dependent on the size of the bubble, so the results are consistent with the results shown in Fig. 4.2 in chapter 4 (the higher the vacuum pressure, the smaller the bubbles). Error bars in Fig. D.3 represent one standard deviation of the amplitude detected from at least 20 bubbles at each vacuum pressure. Even though there is no algorithm to size the bubbles using the detected signals, this result is promising as such an algorithm can be developed.

The results shown in Fig. D.3 are not conclusive because there is no system to verify the size of the detected bubbles. To support the claim that the signals are from micro/nanobubbles, the diluted 
solutions of commercial Definity ${ }^{\circledR}$ microbubbles were used, whose size is known to be distributed in the range of $1-10 \mu \mathrm{m} .(137)$ the signals detected from the Definity ${ }^{\circledR}$ microbubbles were similar in amplitude and frequency of the signals detected from the shrunken bubbles. Further experiments are needed to obtain more definitive conclusions.

In-house generated nanobubble solutions were used to detect signals from individual nanobubbles. The in-house nanobubbles, made of lipid shells and an octafluropropane core, are generated using a protocol published by the group of our collaborator, Dr. Exner's group, at the Case Western Reserve University, Cleveland, OH, United States.(160) A mixture of lipids, 1,2-dipalmitoyl-sn-glycero3-phosphate (DPPA), 1,2-dipalmitoyl-sn-glycero-3-phosphoethanolamine (DPPE), 1,2-dipalmitoyl-snglycero-3- phosphocholine (DPPC) (Corden Pharma Switzerland, Liestal, Switzerland) and 1,2-distearoylsn-glycero-3-phosphoethanolamine-N-[methoxy(polyethylene glycol)-2000] (mPEG-DSPE) (Laysan Bio Inc., Arab, AL, USA) dissolved in propylene glycol is filtered using a syringe filter (Corning Inc. Corning, New York, United States) with a pore size of $0.45 \mu \mathrm{m}$ in order to remove the undissolved lipid particles. The filtered solution is shaken with octafluropropane (C3F8) for 45 seconds in a mechanical shaker (VialMix, Lantheus medical imaging, N. Billerica, MA) to produce solutions of a mixture of micro and nanobubbles that are partitioned using a centrifuge. The partitioned nanobubbles are carefully removed and diluted at 1:10,000 v/v in Phosphate Buffered Saline (PBS).

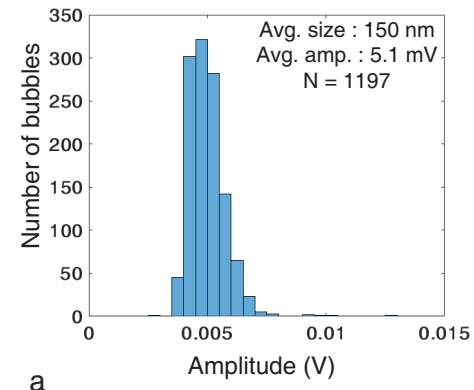

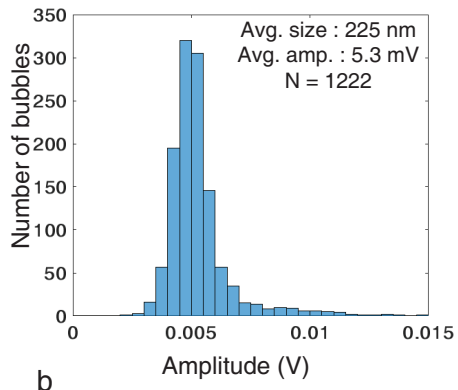

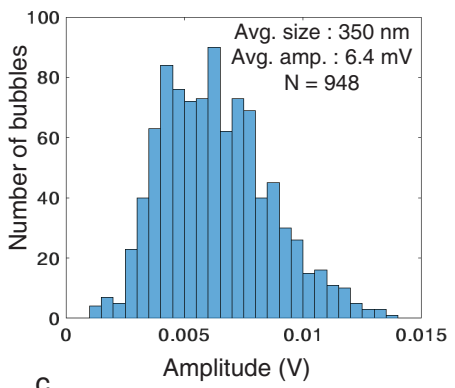

c

Figure D.4: Histograms of the amplitudes of the ultrasound backscatter detected by the acoustic flow cytometer from individual scatterers in the nanobubble solution. These signals were detected from the nanobubbles whose average size, as measured by A are $150 \mathrm{~nm}$ (a), $225 \mathrm{~nm}$ (b), and $350 \mathrm{~nm}$ (c) when measured by the Archimedes ${ }^{\circledR}$ sizer.

\begin{tabular}{l||l|l|l}
\hline \hline Size $(\mathrm{nm})$ & 150 & 225 & 350 \\
\hline Avg. Amplitude $(\mathrm{mV})$ & $5.1 \pm 2.5$ & $5.3 \pm 2.3$ & $6.4 \pm 1.8$ \\
\hline \hline
\end{tabular}

Table D.1: Average amplitude of the signals detected by the flow cytometer for different average size of nanobubbles measured by the Archimedes ${ }^{\circledR}$ sizer.

The histogram of the amplitude of the ultrasound backscatter signals detected from individual 
nanobubbles from three different sample solutions are shown in Fig. D.4. The data presented in the Fig. D.4 is summarized in table D.1. The size of the nanobubbles is measured using the Archimedes ${ }^{\circledR}$. This is one of the first demonstrations of ultrasound signals generated by single nanobubbles.
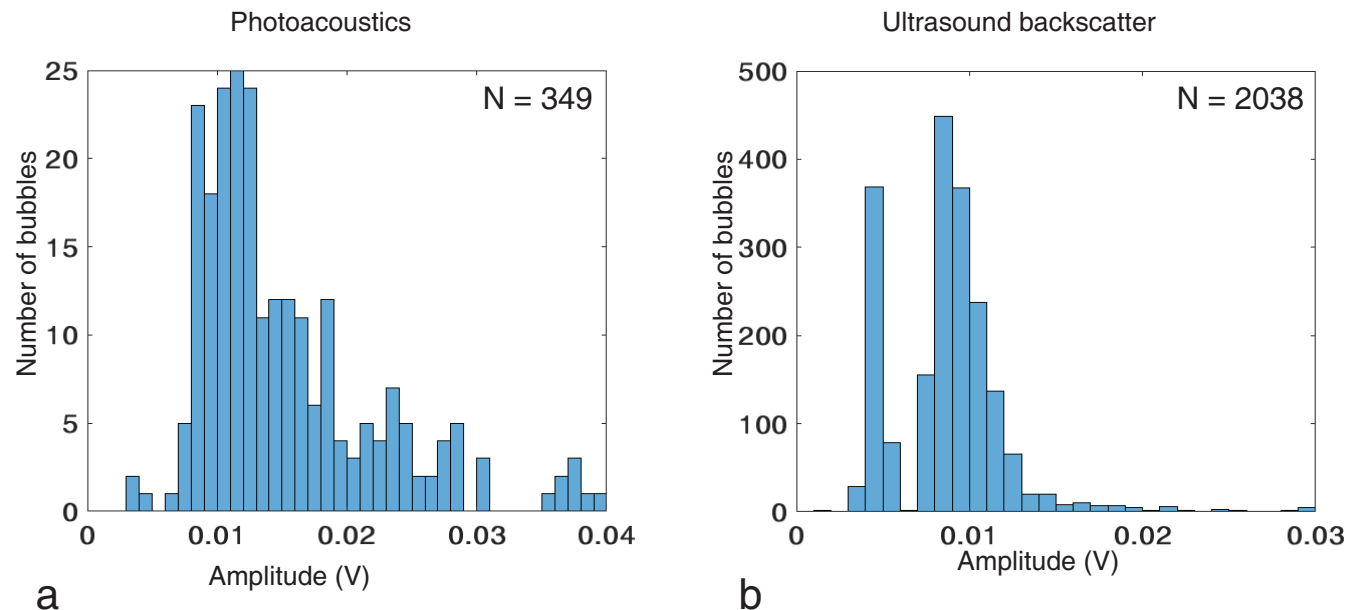

Figure D.5: Histograms of the amplitudes of the ultrasound backscatter and photoacoustic signals simultaneously detected from individual black lipid nanobubbles (dyed using Sudan Black) using the acoustic flow cytometer. These signals were detected from the nanobubbles whose average size is $238 \mathrm{~nm}$ when measured by the Archimedes ${ }^{\mathrm{B}}$ sizer.

Data presented in Fig. D.4 shows only ultrasound backscatter detected from individual nanobubbles. These nanobubbles do not produce photoacoustic signals because they do not absorb sufficient optical energy due to their low optical absorption. Lipid molecules on the shell are only the possible optical absorbers on the nanobubbles and produce photoacoustic signals, which can be used to determine the shell properties. To detect the photoacoustic signals, nanobubbles tagged with Sudan Back B dye (Sigma-Aldrich, St. Louis, MO, USA) were used (which are referred to as black nanobubbles, hereafter). The nanobubble generation protocol is modified to generate black nanobubbles. Similar to the previous experiments, the black nanobubbles were diluted to 1:10,000 v/v in PBS. In this case, the individual black nanobubbles generated both ultrasound backscatter and photoacoustic signals. Fig. D.5 shows the histograms of the amplitude of both ultrasound backscatter (Fig. D.5a) and photoacoustic (Fig. D.5p) signals from each individual black nanobubble. The average diameter of these black nanobubbles is 238 $\mathrm{nm}$, as measured by the Archimedes ${ }^{\circledR}$ instrument.

These experiments demonstrated that the acoustic flow cytometer can be used to detect the signals from individual micro/nanobubbles. Although the system is able to detect individual bubbles, there is no method developed to analyze the detected signals to determine size of the nanobubbles. Developing an algorithm to analyze the signals from individual bubbles can have an impact on research related to micro/nanobubble sizing and characterization. In the future, I will develop algorithms that can be used 
to estimate the size of an individual micro/nanobubble and the shell viscoelastic properties using the ultrasound backscatter and photoacoustic signals. I believe, the acoustic flow cytometer presented in this thesis has potential to overcome the above-mentioned problems related to micro and nanobubble analyses and provide a better platform to further understand the physical properties of micro and nanobubbles. 


\section{Appendix E}

\section{List of publications}

\section{Publications in referred journals}

1. V. Gnyawali, E. M. Strohm, Jun-Zhi Wang, S. S. H. Tsai, and M. C. Kolios, "Microfluidic acoustic and photoacoustic flow cytometry: a label-free approach," Scientific Reports, 2018 (submitted)

2. E. M. Strohm, V. Gnyawali, J. A. Sebastian, R. Ngunjiri, M. J. Moore, S. S. H. Tsai, and M. C. Kolios, Sizing biological cells using a microfluidic acoustic flow cytometer," Lab on a Chip, 2018 (submitted)

3. A. Salari, V. Gnyawali, I. M. Griffiths, R. Karshafian, M. C. Kolios, and S. S. H. Tsai, "Shrinking microbubbles with microfluidics: mathematical modelling to control microbubble sizes," Soft Matter, vol. 13, no. 46, pp. 87968806, 2017.

4. V. Gnyawali, B.-U. Moon, J. Kieda, R. Karshafian, M. C. Kolios, and S. S. H. Tsai, "Honey, I shrunk the bubbles: microfluidic vacuum shrinkage of lipid-stabilized microbubbles," Soft Matter, vol. 13, no. 22, pp. 40114016, 2017.

5. V. Gnyawali, M. Saremi, M. C. Kolios, and S. S. H. Tsai, "Stable microfluidic flow focusing using hydrostatics," Biomicrofluidics, vol. 11, no. 3, p. 34104, 2017.

6. M. Navi, N. Abbasi, M. Jeyhani, V. Gnyawali, S. S. H. Tsai, "Microfluidic diamagnetic waterin-water droplets: a biocompatible cell encapsulation and manipulation platform," Lab on a Chip (submitted)

\section{Patents}

1. S. S. H. Tsai, R. Karshafian, M. C. Kolios, B-U. Moon, and V. Gnyawali, "Apparatus, systems and methods for microfluidic vacuum shrinkage of microbubbles", U. S. Patent Application No. 15/944,453, Filed: Apr. 3, 2018 ; Canadian Patent Application no. 3,000,258, Filed Apr. 4, 2018. 


\section{Publications in conference proceedings}

1. V. Gnyawali, E. M. Strohm, S. S. H. Tsai, and M. C. Kolios, "Simultaneous ultrasound and photoacoustics based flow cytometry," in Proceedings of SPIE, Photons Plus Ultrasound: Imaging and Sensing, 2018, vol. 10494, p. 104940H.

2. V. Gnyawali, J. Kieda, and S. S. H. Tsai, "Microfluidic shrinking of microbubble contrast agents," in Ultrasonics Symposium (IUS), 2017 IEEE International, 2017, pp. 14.

3. E. M. Strohm, V. Gnyawali, M. Van De Vondervoort, Y. Daghighi, S. S. H. Tsai, and M. C. Kolios, "Classification of biological cells using a sound wave based flow cytometer," in Proceedings of SPIE, 2016, vol. 9708, p. 97081A.

4. Y. Daghighi, V. Gnyawali, E. M. Strohm, S. S. H. Tsai, and M. C. Kolios, "One-layer microfluidic device for hydrodynamic 3D self-flow-focusing operating in low flow speed," in Proceedings of SPIE, 2016, vol. 9705 , p. 970505.

\section{Conference abstract and presentations}

1. V. Gnyawali, Eric M. Strohm, Jun-Zhi Wang, S. S. H. Tsai, and M. C. Kolios, "Simultaneous ultrasound backscatter and photoacoustic based flow cytometry," Ontario on a chip 2018, Toronto, Canada (Poster)

2. Morteza Jeyhani, Vaskar Gnyawali, Niki Abbasi, Dae Kun Hwang, and Scott Tsai, "Highthroughput generation of water-in-water droplets in microfluidics platform," Ontario on a chip 2018, Toronto, Canada (Oral)

3. Maryam Navi, Niki Abbasi, Morteza Jeyhani, Vaskar Gnyawali, Scott Tsai, "Diamagnetic separation of cell-encapsulating water-in-water droplets," Ontario on a chip 2018, Toronto, Canada (Poster)

4. V. Gnyawali, B. U. Moon, J. Kieda, R. Karshafian, S. S. H. Tsai, and M. C. Kolios, "Microfluidic shrinking of microbubble contrast agents," IEEE Transactions on Ultrasonics, Ferroelectrics, and Frequency Control, Washington DC, USA (Poster presentation, September 8, 2017)

5. V. Gnyawali, B. U. Moon, J. Kieda, R. Karshafian, S. S. H. Tsai, and M. C. Kolios, "Microfluidic shrinking of microbubble contrast agents," Ontario-on-a-Chip10 Match 2017, Toronto, Canada, May 25, 2017 (Poster)

6. V. Gnyawali, E.M. Strohm, Mia Van de Vondervoort, S.S.H. Tsai, M.C. Kolios, "Microfluidic Three-Dimensional (3D) Flow Focusing for Acoustics Based Cellular Cytometry", MRS Spring Meeting, Phoenix, Arizona, USA, May 27, 2016 (Poster)

7. Y. Daghighi, E. M. Strohm,V. Gnyawali, M. Moore, S. S. H. Tsai, and M. C. Kolios. (2016). "A hydrodynamically tunable microfluidic 3D flow-focusing device" Ontario-on-a-Chip10 Match 2016, Toronto, Canada, May 27, 2016 (Poster) 
8. E. M. Strohm, V. Gnyawali, P. Saggu, Y. Daghighi, S. S. H. Tsai, and M. C. Kolios, "Cell sizing using acoustic flow cytometry," Ontario-on-a-Chip10 Match 2015, Toronto, Canada, May 27, 2016 (First prize winner, Oral competition)

9. V. Gnyawali, E.M. Strohm, Mia Van De Vondervoort, Y. Daghighi, M.C. Kolios, S.S.H. Tsai, "Ultrasound and photoacoustic based flow cytometer", RTC poster competition, St. Michaels Hospital, Canada, April, 2016 (Poster)

10. E. M. Strohm, V. Gnyawali, M. Van De Vondervoort, Y. Daghighi, S. S. H. Tsai, and M. C. Kolios, "Classification of biological cells using a sound wave based flow cytometer," SPIE Proceedings 2016, San Francisco, California, USA, February 2016 (Oral)

11. Y. Daghighi, V. Gnyawali, E. M. Strohm, S. S. H. Tsai, and M. C. Kolios, "One-layer microfluidic device for hydrodynamic 3D self-flow-focusing operating in low flow speed," SPIE Proceedings 2016, San Francisco, California, USA, February 2016 (Oral)

12. V. Gnyawali, E.M. Strohm, Y. Daghighi, Mia Van de Vondervoort, S.S.H. Tsai, M.C. Kolios, "3D flow focusing for microfluidic flow cytometry with ultrasonics", 68th Annual Meeting of the APS Division of Fluid Dynamics, Boston, Massachusetts, USA, November 2015 (Oral)

13. E.M. Strohm, V. Gnyawali, Y. Daghighi, S.S.H. Tsai, M.C. Kolios, "Sound wave based flow cytometry for tumor cell detection", 2015 Canadian Cancer Research Conference, Montreal, Canada,Nov 8-10, 2015 (Poster)

14. E.M. Strohm, V. Gnyawali, P. Saggu, Y. Daghighi, S.S.H. Tsai, M.C. Kolios, "Development of a sound wave based flow cytometer", Ontario-on-a-Chip10 Match 2015, Toronto, Canada, May 28, 2015 (Poster) 



\section{Bibliography}

[1] B. G. Chung, J. W. Park, J. S. Hu, C. Huang, E. S. Monuki, and N. L. Jeon. A hybrid microfluidicvacuum device for direct interfacing with conventional cell culture methods. BMC Biotechnology, 7:60, 2007.

[2] H. M. Davey and D. B. Kell. Flow cytometry and cell sorting of heterogeneous microbial populations: the importance of single-cell analyses. Microbiological reviews, 60(4):641-96, 1996.

[3] M. I. Lapsley, L. Wang, and T. J. Huang. On-chip flow cytometry: where is it now and where is it going? Biomarkers in Medicine, 7(1):75-78, 2013.

[4] K. Cheung, S. Gawad, and P. Renaud. Impedance spectroscopy flow cytometry: On-chip label-free cell differentiation. Cytometry Part A, 65(2):124-132, 2005.

[5] J. A. Simpson. Counting and Sizing of Submicron Particles by the Resistive Pulse Technique. Review of Scientific Instruments, 41(7):909-916, 1970.

[6] S. Gawad, L. Schild, and P. Renaud. Micromachined impedance spectroscopy flow cytometer for cell analysis and particle sizing. Lab on a Chip, 1(1):76-82, 2001.

[7] C. Giesecke, K. Feher, K. von Volkmann, J. Kirsch, A. Radbruch, and T. Kaiser. Determination of background, signal-to-noise, and dynamic range of a flow cytometer: A novel practical method for instrument characterization and standardization. Cytometry Part A, 91A:1104-1114, 2017.

[8] H-W Wang, N Bao, T. L. Le, C. Lu, and J-X Cheng. Microfluidic CARS cytometry. Optics Express, 16(8):5782, 2008.

[9] Y. Zheng, J. Nguyen, Y. Wei, and Y. Sun. Recent advances in microfluidic techniques for single-cell biophysical characterization. Lab on a Chip, 13(13):2464, 2013.

[10] M. Xu and L. V. Wang. Photoacoustic imaging in biomedicine. Review of Scientific Instruments, 77(4):041101, 2006.

[11] M. J . K. Blomley, J. C. Cooke, E. C. Unger, M. J. Monaghan, and D. O. Cosgrove. Microbubble contrast agents : a new era in ultrasound Clinical review Microbubble contrast agents : a new era in ultrasound. British Medical Journal, 322(7296):1222-1225, 2001.

[12] M. Postema and G. Schmitz. Ultrasonic bubbles in medicine: Influence of the shell. Ultrasonics Sonochemistry, 14(4):438-444, 2007. 
[13] S. Qin, C. F. Caskey, and K. W. Ferrara. Ultrasound contrast microbubbles in imaging and therapy: physical principles and engineering. Physics in Medicine and Biology, 54(6):1-42, 2009.

[14] S. M. van der Meer, B. Dollet, M. M. Voormolen, C. T. Chin, A. Bouakaz, N. de Jong, M. Versluis, and D. Lohse. Microbubble spectroscopy of ultrasound contrast agents. The Journal of the Acoustical Society of America, 121(1):648-656, 2007.

[15] H. Lin, J. Chen, and C. Chen. A novel technology: microfluidic devices for microbubble ultrasound contrast agent generation. Medical and Biological Engineering and Computing, 54(9):1$14,2016$.

[16] K. Hettiarachchi, E. Talu, M. L Longo, P. A. Dayton, and A. P Lee. On-chip generation of microbubbles as a practical technology for manufacturing contrast agents for ultrasonic imaging. Lab on a Chip, 7(4):463-468, 2007.

[17] M. Seo, R. Williams, and N. Matsuura. Size reduction of cosolvent-infused microbubbles to form acoustically responsive monodisperse perfluorocarbon nanodroplets. Lab on a Chip, 15(17):3581-3590, 2015.

[18] J. Sijl, E. Gaud, P. J. A. Frinking, M. Arditi, N. de Jong, D. Lohse, and M. Versluis. Acoustic characterization of single ultrasound contrast agent microbubbles. The Journal of the Acoustical Society of America, 124(6):4091-4097, 2008.

[19] D. Maresca, M. Emmer, P. L. M. J. van Neer, H. J. Vos, M. Versluis, M. Muller, N. de Jong, and A. F. W. van der Steen. Acoustic Sizing of an Ultrasound Contrast Agent. Ultrasound in Medicine and Biology, 36(10):1713-1721, 2010.

[20] Lenitza M Nieves, Christopher Hernandez, Judy Hadley, and Robert Coyne. Effect of the Surfactant Pluronic on the Stability of. In Ultrasonics Symposium (IUS), IEEE, pages 8-11, 2017.

[21] M. Mancini, A. Greco, A. Speranza, and M. Salvatore. Ultrasound molecular imaging with targeted microbubble contrast agents. J. Nucl. Cardiol., 14:876-884, 2007.

[22] J. Ducree and R. Zengerle. Microfluidics. Springer, 2004.

[23] H. Bruus. Acoustofluidics 1: Governing equations in microfluidics. Lab on a Chip, 11(22):3742, 2011.

[24] S. Tripathi, A. Kumar, Y. V. Bala Varun Kumar, and A. Agrawal. Three-dimensional hydrodynamic flow focusing of dye, particles and cells in a microfluidic device by employing two bends of opposite curvature. Microfluidics and Nanofluidics, 20(2):1-14, 2016.

[25] J. Knight, A. Vishwanath, J. Brody, and R. Austin. Hydrodynamic Focusing on a Silicon Chip: Mixing Nanoliters in Microseconds. Physical Review Letters, 80(17):3863-3866, 1998.

[26] C. Simonnet and A. Groisman. High-throughput and high-resolution flow cytometry in molded microfluidic devices. Analytical Chemistry, 78(16):5653-63, aug 2006.

[27] J. P. Golden, G. A. Justin, M. Nasir, and F. S. Ligler. Hydrodynamic focusing a versatile tool. Analytical and Bioanalytical Chemistry, 402(1):325-335, 2012. 
[28] A. A. Nawaz, X. Zhang, X. Mao, J. Rufo, S. S. Lin, F. Guo, Y. Zhao, M. Lapsley, P. Li, J. P. McCoy, S. J. Levine, and T. J. Huang. Sub-micrometer-precision, three-dimensional (3D) hydrodynamic focusing via "microfluidic drifting". Lab on a Chip, 14(2):415-23, 2014.

[29] K. T. Dussik. Über die Möglichkeit, hochfrequente mechanische Schwingungen als diagnostisches Hilfsmittel zu verwerten. Zeitschrift für die gesamte Neurologie und Psychiatrie, 174, 1942.

[30] F. Tranquart, N. Grenier, V. Eder, and L. Pourcelot. Clinical Use of Ultrasound Tissue Harmonic Imaging. Ultrasound in medicine $\&$ biology, 25(6):889-894, 1999.

[31] F. L. Lizzi, M. Greenebaum, E. J. Feleppa, M. Elbaum, and D. J. Coleman. Theoretical framework for spectrum analysis in ultrasonic tissue characterization. The Journal of the Acoustical Society of America, 73(4):1366-1373, 1983.

[32] G. J. Czarnota, M. C. Kolios, H. Vaziri, F. P. Ottensmeyer, M. D. Sherar, and J. W. Hunt. Ultrasonic biomicroscopy of viable, dead and apoptotic cells. Ultrasound in Medicine $\mathscr{E}$ Biology, 23(6):961-965, 1997.

[33] F. S. Foster, J. Mehi, M. Lukacs, D. Hirson, C. White, C. Chaggares, and A. Needles. A New 15-50 $\mathrm{MHz}$ Array-Based Micro-Ultrasound Scanner for Preclinical Imaging. Ultrasound in Medicine and Biology, 35(10):1700-1708, 2009.

[34] R. G. Maev. Acoustic Microscopy: Fundamentals and Applications. John Wiley \& Sons, 2008.

[35] A. Briggs and O. Kolosov. Acoustic microscopy. Oxford University Press, 2009.

[36] M. C. Kolios. Biomedical ultrasound imaging from 1 to 1000 MHz. Canadian Acoustics, 37(3):3543, 2009.

[37] R. A. Lemons and C. F. Quate. Acoustic microscope - Scanning version. Applied Physics Letters, 24(4):163-165, 1974.

[38] J. Yao and L. V. Wang. Photoacoustic Microscopy. Laser Photon Review, 7(5):1-36, 2014.

[39] K. Wilson, K. Homan, and S. Emelianov. Biomedical photoacoustics beyond thermal expansion using triggered nanodroplet vaporization for contrast-enhanced imaging. Nature Communications, 3:618, 2012.

[40] G. J. Diebold, M. I. Khan, and S. M. Park. Photoacoustic "signatures" of particulate matter: optical production of acoustic monopole radiation. Science (New York, N.Y.), 250(4977):101104, 1990.

[41] G. J. Diebold. Photoacoustic monopole radiation: waves from objects with symmetry in one, two and three dimensions. Photoacoustic Imaging and Spectroscopy, pages 3-17, 2009.

[42] E. Hysi, E. M. Strohm, and M. C. Kolios. Probing Different Biological Length Scales Using Photoacoustics: From 1 To $1000 \mathrm{MHz}$. In Handbook of Photonics for Biomedical Engineering, pages 1-18. 2014.

[43] E. M. Strohm, E. Hysi, and M. C. Kolios. Photoacoustic measurements of single red blood cells. In 2012 IEEE International Ultrasonics Symposium, pages 1406-1409, 2012. 
[44] E. M. Strohm, E. S. L. Berndl, and M. C. Kolios. High frequency label-free photoacoustic microscopy of single cells. Photoacoustics, 1(3-4):49-53, dec 2013.

[45] L. V. Wang and H. Wu. Biomedical Optics: Principles and Imaging. John Wiley \& Sons, 2007.

[46] G. Paltauf and P. E. Dyer. Photomechanical processes and effects in ablation. Chemical Reviews, 103(2):487-518, 2003.

[47] I. G. Calasso, W. Craig, and G. J. Diebold. Photoacoustic point source. Physical Review Letters, 86(16):3550-3553, 2001.

[48] G. J. Diebold. The photoacoustic effect generated isotropic solid sphere. 33(4):265-269, 1995.

[49] L. V. Wang. Ultrasound-mediated biophotonic imaging: A review of acousto-optical tomography and photo-acoustic tomography. Disease Markers, 19(2-3):123-138, 2004.

[50] K. K. Shung. Diagnostic ultrasound: Past, present, and future. Journal of Medical and Biological Engineering, 31(6):371-374, 2011.

[51] V. Gnyawali, M. Saremi, M. C. Kolios, and S. S. H. Tsai. Stable microfluidic flow focusing using hydrostatics. Biomicrofluidics, 11(3):034104, 2017.

[52] T. Segers, L. De Rond, N. De Jong, M. Borden, and M. Versluis. Stability of Monodisperse Phospholipid-Coated Microbubbles Formed by Flow-Focusing at High Production Rates. Langmuir, 32(16):3937-3944, 2016.

[53] S. Unnikrishnan and A. L. Klibanov. Microbubbles as ultrasound contrast agents for molecular imaging: Preparation and application. American Journal of Roentgenology, 199(2):292-299, 2012.

[54] R. Karshafian, P. D. Bevan, R. Williams, S. Samac, and P. N. Burns. Sonoporation by UltrasoundActivated Microbubble Contrast Agents: Effect of Acoustic Exposure Parameters on Cell Membrane Permeability and Cell Viability. Ultrasound in Medicine and Biology, 35(5):847-860, 2009.

[55] P. Prentice, P. Cuschieri, K. Dholakia, M. Prausnitz, and P. Campbell. Membrane disruption by optically controlled microbubble cavitation. Nature Physics, 1(2):107-110, 2005.

[56] R. E. Apfel and C. K. Holland. Gauging the likelihood of cavitation from short-pulse, low-duty cycle diagnostic ultrasound. Ultrasound in Medicine and Biology, 17(2):179-185, 1991.

[57] E. C. Unger, T. O. Matsunaga, T. McCreery, P. Schumann, R. Sweitzer, and R. Quigley. Therapeutic applications of microbubbles. European Journal of Radiology, 42(2):160-168, 2002.

[58] R. Bekeredjian, P. A. Grayburn, and R. V. Shohet.

[59] R. J. Price, D. M. Skyba, S. Kaul, and T. C. Skalak. Delivery of colloidal particles and red blood cells to tissue through microvessel ruptures created by targeted microbubble destruction with ultrasound. Circulation, 98(13):1264-1267, 1998.

[60] P. A. Auroux, D. Iossifidis, D. R. Reyes, and A. Manz. Micro total analysis systems. 2. Analytical standard operations and applications. Analytical Chemistry, 74(12):2637-2652, 2002. 
[61] A. Manz, H. M. Widmers, and N. Graber. Miniaturized total chemical analysis systems: A novel concept for chemical sensing. Sensors and Actuators B: Chemical, 1(1-6):244-248, 1990.

[62] D. Mark, S. Haeberle, G. Roth, F. von Stetten, and R. Zengerle. Microfluidic lab-on-a-chip platforms: requirements, characteristics and applications. Chemical Society Reviews, 39(3):1153, 2010.

[63] L. J. Millet, J. D. Lucheon, R. F. Standaert, S. T. Retterer, and M. J. Doktycz. Modular microfluidics for point-of-care protein purifications. Lab on a Chip, 15(8):1799-1811, 2015.

[64] H. A. Stone, A. D. Stroock, and A. Ajdari. Engineering Flows in Small Devices: Microfluidics Toward a Lab-on-a-Chip. Annual Review of Fluid Mechanics, 36(1):381-411, 2004.

[65] S-I Pai. Viscous flow theory: Laminar flow. D. Van Nostrand, 1956.

[66] A. S. Yang and W. H. Hsieh. Hydrodynamic focusing investigation in a micro-flow cytometer. Biomedical Microdevices, 9(2):113-122, 2007.

[67] G-B Lee, C-I Hung, B-J Ke, G-R Huang, B-H Hwei, and H-F Lai. Hydrodynamic Focusing for a Micromachined Flow Cytometer. Journal of Fluids Engineering, 123(3):672, 2001.

[68] J. V. Watson. Introduction to flow cytometry. 2004.

[69] M. Brown and C. Wittwer. Flow Cytometry: Principles and Clinical Applications in Hematology. Clinical Chemistry, 46(8):1221-1229, 2000.

[70] T. Sun and H. Morgan. Single-cell microfluidic Impedance cytometry: A review. Microfluidics and Nanofluidics, 8(4):423-443, 2010.

[71] Q. Xu, M. Hashimoto, T. T. Dang, T. Hoare, D. S. Kohane, G. M. Whitesides, R. Langer, and D. G. Anderson. Preparation of Monodisperse Biodegradable Polymer Microparticles Using a Microfluidic Flow-Focusing Device for Controlled Drug Delivery. Small, 02115(13):1575-1581, 2009.

[72] P. S. Dittrich and A. Manz. Lab-on-a-chip: microfluidics in drug discovery. Nature Reviews Drug Discovery, 5(3):210-218, 2006.

[73] P. K. Wong, Y-K Lee, and C-M Ho. Deformation of DNA molecules by hydrodynamic focusing. Journal of Fluid Mechanics, 497:55-65, 2003.

[74] T. Ward, M. Faivre, M. Abkarian, and H. A. Stone. Microfluidic flow focusing: Drop size and scaling in pressure versus flow-rate-driven pumping. Electrophoresis, 26(19):3716-3724, 2005.

[75] Z. Li, S. Y. Mak, . Sauret, and H. C. Shum. Syringe-pump-induced fluctuation in all-aqueous microfluidic system implications for flow rate accuracy. Lab on a Chip, 14(4):744, 2014.

[76] W. Zeng, I. Jacobi, D. J. Beck, S. Li, and H. A. Stone. Characterization of syringe-pump-driven induced pressure fluctuations in elastic microchannels. Lab on a Chip, 15(February):1110-1115, 2015.

[77] J. Atencia and D. J. Beebe. Steady flow generation in microcirculatory systems. Lab on a Chip, 6(4):567-74, 2006. 
[78] X. Mao, S-C S. Lin, C. Dong, and T. J. Huang. Single-layer planar on-chip flow cytometer using microfluidic drifting based three-dimensional (3D) hydrodynamic focusing. Lab on a Chip, 9(11):1583-1589, 2009.

[79] E. M. Strohm, M. J. Moore, and M. C. Kolios. Single Cell Photoacoustic Microscopy: A Review. IEEE Journal on Selected Topics in Quantum Electronics, 22(3), 2016.

[80] Y. Xia and G. M. Whitesides. Soft Lithography. Annual Review of Materials Science, 28(1):153$184,1998$.

[81] N. Otsu. A threshold selection method from gray-level histograms. IEEE Transactions on Systems, Man, and Cybernetics, 9(1):62-66, 1979.

[82] T. Stiles, R. Fallon, T. Vestad, J. Oakey, D. W M Marr, J. Squier, and R. Jimenez. Hydrodynamic focusing for vacuum-pumped microfluidics. Microfluidics and Nanofluidics, 1(3):280-283, 2005.

[83] D. H. Tycko, M. H. Metz, E. A. Epstein, and A. Grinbaum. Flow-cytometric light scattering measurement of red blood cell volume and hemoglobin concentration. Applied Optics, 24(9):1355$1365,1985$.

[84] M. J. Skogen-hagenson, G. C. Salzman, P. F. Mullaney, and W. H. Brockman. A high efficiency Flow Cytometer. The Journal of Histochemistry and Cytochemistry, 25(7):784-789, 1977.

[85] M. D. Berardino. Electrical Detection in Microfluidic Flow Cytometers. In The Microflow Cytometer. Pan Stanford Publishing Pte. Ltd., 2010.

[86] R. R. Jahan-tigh, C. Ryan, G Obermoser, and K. Schwarzenberger. Flow Cytometry. Journal of Investigative Dermatology, 132(10):1-6, 2012.

[87] H. M. Shapiro. The evolution of cytometers. Cytometry Part A, 58A(1):13-20, 2004.

[88] A. Adan, G. Alizada, Y. Kiraz, Y. Baran, and A. Nalbant. Flow cytometry: basic principles and applications. Critical Reviews in Biotechnology, 37(2):163-176, 2017.

[89] O. A. Saleh and L. L. Sohn. Quantitative sensing of nanoscale colloids using a microchip Coulter counter. Review of Scientific Instruments, 72(12):4449-4451, 2001.

[90] M. E. Piyasena and S. W. Graves. The intersection of flow cytometry with microfluidics and microfabrication. Lab on a Chip, 14(6):1044-1059, 2014.

[91] H-S Liu, M-S Jan, C-K Chou, P-H Chen, and N-J Ke. Is Green Fluorescent Protein Toxic to the Living Cells? Biochemical and Biophysical Research Communications, 260(3):712-717, 1999.

[92] T. T. Yang, L. Cheng, and S. R. Kain. Optimized codon usage and chromophore mutations provide enhanced sensitivity with the green fluorescent protein. Nucleic Acids Research, 24(22):45924593, 1996.

[93] M-H Sung. A Checklist for Successful Quantitative Live Cell Imaging in Systems Biology. Cells, 2(2):284-293, 2013. 
[94] Y. Komatsu, R. Nagaoka, K. Funamoto, T. Hayase, N. Masauzi, H. Kanai, and Y. Saijo. Sonocytometry Novel Diagnostic Method of Ultrasonic Differentiation of Cells in Blood Flow. In Engineering in Medicine and Biology Society (EMBC), pages 2761-2764, 2014.

[95] E. M. Strohm, M. C. Kolios, D. K. Hwang, B-U Moon, and S. S. H. Tsai. Development of a microfluidic device with integrated high frequency ultrasound probe for particle characterization. In IEEE International Ultrasonics Symposium, IUS, pages 1960-1963, 2014.

[96] R. E. Baddour, M. D. Sherar, J. W. Hunt, G. J. Czarnota, and M. C. Kolios. High-frequency ultrasound scattering from microspheres and single cells. The Journal of the Acoustical Society of America, 117(2):934-943, 2005.

[97] O. Falou, M. Rui, A. El Kaffas, J. C. Kumaradas, and M. C. Kolios. The measurement of ultrasound scattering from individual micron-sized objects and its application in single cell scattering. The Journal of the Acoustical Society of America, 128(2):894-902, 2010.

[98] V. C. Anderson. Sound Scattering from a Fluid Sphere. The Journal of the Acoustical Society of America, 22(4), 1950.

[99] G. J. Diebold, T. Sun, and M. I. Khan. Photoacoustic Monopole Radiation in 1,2 and 3D, 1991.

[100] E. Strohm, G. J. Czarnota, and M. C. Kolios. Quantitative measurements of apoptotic cell properties using acoustic microscopy. IEEE Transactions on Ultrasonics, Ferroelectrics, and Frequency Control, 57(10):2293-2304, 2010.

[101] C. Zhang, K. Maslov, and L. V. Wang. Subwavelength-resolution label-free photoacoustic microscopy of optical absorption in vivo. Optics Letters, 35(19):3195-3197, 2010.

[102] E. M. Strohm and M. C. Kolios. Classification of blood cells and tumor cells using label-free ultrasound and photoacoustics. Cytometry Part A, 87(8):741-749, 2015.

[103] M. N. Fadhel, E. S.L. Berndl, E. M. Strohm, and M. C. Kolios. High-Frequency Acoustic Impedance Imaging of Cancer Cells. Ultrasound in Medicine and Biology, 41(10):2700-2713, 2015.

[104] M. M. Pasternak, E. M. Strohm, E. S. L. Berndl, and M.l C. Kolios. Properties of cells through life and death - an acoustic microscopy investigation. Cell Cycle, 14(18):2891-2898, 2015.

[105] C. P. Favazza, L. A. Cornelius, and L. V. Wang. In vivo functional photoacoustic microscopy of cutaneous microvasculature in human skin. Journal of Biomedical Optics, 16(2):026004, mar 2011.

[106] M. J. Moore, E. M. Strohm, and M. C. Kolios. Assessment of the Nucleus-to-Cytoplasmic Ratio in MCF-7 Cells Using Ultra-high Frequency Ultrasound and Photoacoustics. International Journal of Thermophysics, 37(12):1-10, 2016.

[107] Abcam. Flow Cytometry Whole Blood Samples : Red Blood Cell Lysis, 2005.

[108] A. L. Landay and K. A. Muirhead. Procedural guidelines for performing immunophenotyping by flow cytometry. Clinical Immunology and Immunopathology, 52(1):48-60, 1989. 
[109] P. Renzi and L. C. Ginns. Analysis of T cell subsets in normal adults. Journal of Immunological Methods, 98(1):53-56, 1987.

[110] J. Vuorte, S. E. Jansson, and H. Repo. Evaluation of Red Blood Cell Lysing Solutions in the Study of Neutrophil Oxidative Burst by the DCFH Assay. Cytometry, 43(4):290-296, 2001.

[111] P. Sethu, M. Anahtar, L. L. Moldawer, R. G. Tompkins, and M. Toner. Continuous flow microfluidic device for rapid erythrocyte lysis. Analytical Chemistry, 76(21):6247-6253, 2004.

[112] G. A. Webster and A. G. Pockley. Effect of red cell lysis protocols on the expression of rat peripheral blood lymphocyte subset and activation antigens. Journal of Immunological Methods, 163(1):115-21, 1993.

[113] L. W. M. M. Terstappen, H. Meiners, and M. R. Loken. A rapid sample preparation technique for flow cytometric analysis of immunofluorescence allowing absolute enumeration of cell subpopulations. Journal of Immunological Methods, 123(1):103-112, 1989.

[114] K. Galler, K. Bräutigam, C. Große, J. Popp, and U. Neugebauer. Making a big thing of a small cell recent advances in single cell analysis. The Analyst, 139(6):1237-1273, 2014.

[115] G. He, D. Xu, H. Qin, S. Yang, and D. Xing. In vivo cell characteristic extraction and identification by photoacoustic flow cytography. Biomedical Optics Express, 6(10):3748, 2015.

[116] S. Takeuchi, P. Garstecki, D. B. Weibel, and G. M. Whitesides. An axisymmetric flow-focusing microfluidic device. Advanced Materials, 17(8):1067-1072, 2005.

[117] P. A. Dayton, K. E. Morgan, A. L. Klibanov, G. H. Brandenburger, and K. W. Ferrara. Optical and acoustical observations of the effects of ultrasound on contrast agents. IEEE Transactions on Ultrasonics, Ferroelectrics, and Frequency Control, 46(1):220-232, 1999.

[118] J. Ma, C. Xu, F. Gao, M. Chen, F. Li, and L. Du. Diagnostic and therapeutic research on ultrasound microbubble/nanobubble contrast agents (Review). Molecular Medicine Reports, 12:4022-4028, 2015.

[119] J. E. Streeter, R. Gessner, I. Miles, and P. A. Dayton. Improving Sensitivity in Ultrasound Molecular Imaging by Tailoring Contrast Agent Size Distribution: In Vivo Studies Jason. Molecular Imaging, 9(2):87-95, 2010.

[120] S. Sirsi and M. Borden. Microbubble Compositions, Properties and Biomedical Applications. Bubble Science, Engineering \& Technology, 1:3-17, 2010.

[121] R. V. Shohet, S. Chen, Y. T. Zhou, Z. Wang, R. S. Meidell, R. H. Unger, and P. A. Grayburn. Echocardiographic destruction of albumin microbubbles directs gene delivery to the myocardium. Circulation, 101(22):2554-2556, 2000.

[122] E. Stride and M. Edirisinghe. Special issue on microbubbles: From contrast enhancement to cancer therapy. Medical and Biological Engineering and Computing, 47(8):809-811, 2009.

[123] R. Karshafian, S. Samac, P. D. Bevan, and P. N. Burns. Microbubble mediated sonoporation of cells in suspension: Clonogenic viability and influence of molecular size on uptake. Ultrasonics, 50(7):691-697, 2010. 
[124] P. Marmottant and S. Hilgenfeldt. Controlled vesicle deformation and lysis by single oscillating bubbles. Nature, 423(6936):153-156, 2003.

[125] F. Fekri, R. C. Delos Santos, R. Karshafian, and C. N. Antonescu. Ultrasound microbubble treatment enhances clathrin-mediated endocytosis and fluid-phase uptake through distinct mechanisms. PLoS ONE, 11(6):1-22, 2016.

[126] N. Rapoport, Z. Gao, and A. Kennedy. Multifunctional nanoparticles for combining ultrasonic tumor imaging and targeted chemotherapy. Journal of the National Cancer Institute, 99(14):1095$1106,2007$.

[127] Z. Gao, A. M. Kennedy, D. A. Christensen, and N. Y. Rapoport. Drug-loaded nano/microbubbles for combining ultrasonography and targeted chemotherapy. Ultrasonics, 48(4):260-270, 2008.

[128] J. R. Lindner. Microbubbles in medical imaging: current applications and future directions. Nature Reviews Drug Discovery, 3(6):527-533, 2004.

[129] E. Stride and M. Edirisinghe. Novel microbubble preparation technologies. Soft Matter, 4:2350$2359,2008$.

[130] K. Bjerknes, K. Dyrstad, G. Smistad, and I. Agerkvist. Preparation of polymeric microcapsules: formulation studies. Drug Development and Industrial Pharmacy, 26(8):847-56, 2000.

[131] M. Kukizaki and M. Goto. Spontaneous formation behavior of uniform-sized microbubbles from Shirasu porous glass (SPG) membranes in the absence of water-phase flow. Colloids and Surfaces A: Physicochemical and Engineering Aspects, 296(1-3):174-181, 2007.

[132] U. Farook, H. B. Zhang, M. J. Edirisinghe, E. Stride, and N. Saffari. Preparation of microbubble suspensions by co-axial electrohydrodynamic atomization. Medical Engineering and Physics, 29(7):749-754, 2007.

[133] A. H. Dhanaliwala, J. L. Chen, S. Wang, and J. A. Hossack. Liquid flooded flow-focusing microfluidic device for in situ generation of monodisperse microbubbles. Microfluidics and Nanofluidics, 14(3-4):457-467, 2013.

[134] H. Chen, J. Li, W. Zhou, E. G. Pelan, S. D. Stoyanov, L. N. Arnaudov, and H. A. Stone. Sonicationmicrofluidics for fabrication of nanoparticle-stabilized microbubbles. Langmuir, 30(15):42624266, 2014.

[135] S. A. Peyman, J. R. McLaughlan, R. H. Abou-Saleh, G. Marston, B. R. G. Johnson, S. Freear, P. L. Coletta, A. F. Markham, and S. D. Evans. On-chip preparation of nanoscale contrast agents towards high-resolution ultrasound imaging. Lab on a Chip, 16(4):679-687, 2016.

[136] C. Lochovsky, S. Yasotharan, and A. Günther. Bubbles no more: in-plane trapping and removal of bubbles in microfluidic devices. Lab on a Chip, 12(3):595, 2012.

[137] Definity. Lantheus Medical Imagin g. Definity (perflutren lipid microsphere) injectable suspension.

[138] E. Y. Arashiro and N. R. Demarquette. Use of the pendant drop method to measure interfacial tension between molten polymers. Materials Research, 2(1):23-32, 1999. 
[139] P. S. Sheeran, Y. Daghighi, K. Yoo, R. Williams, E. Cherin, F. S. Foster, and P. N. Burns. ImageGuided Ultrasound Characterization of Volatile Sub-Micron Phase-Shift Droplets in the 20-40 MHz Frequency Range. Ultrasound in Medicine and Biology, 42(3):795-807, 2016.

[140] B-U Moon, D. K. Hwang, and S. S. H. Tsai. Shrinking, growing, and bursting: microfluidic equilibrium control of water-in-water droplets. Lab on a Chip, 8(14):198-220, 2016.

[141] T. Cubaud, M. Sauzade, and R. Sun. CO2 dissolution in water using long serpentine microchannels. Biomicrofluidics, 6(2):1-9, 2012.

[142] S. Shim, J. Wan, S. Hilgenfeldt, P. D. Panchal, and H. A. Stone. Dissolution without disappearing: multicomponent gas exchange for $\mathrm{CO} 2$ bubbles in a microfluidic channel. Lab on a Chip, 14(14):2428-36, 2014.

[143] D. J. Cox and J. L. Thomas. Rapid Shrinkage of Lipid-Coated Bubbles in Pulsed Ultrasound. Ultrasound in Medicine and Biology, 39(3):466-474, 2013.

[144] T. C. Merkel, V. I. Bondar, K. Nagai, B. D. Freeman, and I. Pinnau. Gas sorption, diffusion, and permeation in poly(dimethylsiloxane). Journal of Polymer Science Part B: Polymer Physics, 38(3):415-434, 2000.

[145] L. Xu, H. Lee, D. Jetta, and K. W. Oh. Vacuum-driven power-free microfluidics utilizing the gas solubility or permeability of polydimethylsiloxane (PDMS). Lab on a Chip, 15(20):3962-3979, 2015.

[146] J. A. Swanson, M. Lee, and P. E. Knapp. Cellular dimensions affecting the nucleocytoplasmic volume ratio. Journal of Cell Biology, 115(4):941-948, 1991.

[147] H. Mulvana, R. J. Browning, Y. Luan, N. De Jong, M. X. Tang, R. J. Eckersley, and E. Stride. Characterization of contrast agent microbubbles for ultrasound imaging and therapy research. IEEE Transactions on Ultrasonics, Ferroelectrics, and Frequency Control, 64(1):232-251, 2017.

[148] Z. Nie, M. Seo, S. Xu, P. C. Lewis, M. Mok, E. Kumacheva, G. M. Whitesides, P. Garstecki, and H. A. Stone. Emulsification in a microfluidic flow-focusing device: Effect of the viscosities of the liquids. Microfluidics and Nanofluidics, 5(5):585-594, 2008.

[149] E. Cosatto, M. Miller, H. P. Graf, and J. S. Meyer. Grading nuclear pleomorphism on histological micrographs. 2008 19th International Conference on Pattern Recognition, pages 1-4, 2008.

[150] S. Petushi, F. U. Garcia, M. M. Haber, C. Katsinis, and A. Tozeren. Large-scale computations on histology images reveal grade-differentiating parameters for breast cancer. BMC Medical Imaging, 6:1-11, 2006.

[151] V. Nandakumar, L. Kelbauskas, K. F. Hernandez, K. M. Lintecum, P. Senechal, K. J. Bussey, P. C. W. Davies, R. H. Johnson, and D. R. Meldrum. Isotropic 3D nuclear morphometry of normal, fibrocystic and malignant breast epithelial cells reveals new structural alterations. PLoS ONE, 7(1), 2012. 
[152] C. A. Sennoga, V. Mahue, J. Loughran, J. Casey, J. M. Seddon, M. Tang, and R. J. Eckersley. On sizing and counting of microbubbles using optical microscopy. Ultrasound in Medicine and Biology, 36(12):2093-2096, 2010.

[153] S. J. Satinover, J. D. Dove, and M. A. Borden. Single-particle optical sizing of microbubbles. Ultrasound in Medicine and Biology, 40(1):138-147, 2014.

[154] C. A. Sennoga, J. S. M. Yeh, J. Alter, E. Stride, P. Nihoyannopoulos, J. M. Seddon, D. O. Haskard, J. V. Hajnal, M. X. Tang, and R. J. Eckersley. Evaluation of Methods for Sizing and Counting of Ultrasound Contrast Agents. Ultrasound in Medicine and Biology, 38(5):834-845, 2012.

[155] E. Y. Lukianova-Hleb, A. Belyanin, S. Kashinath, X. Wu, and D. O. Lapotko. Plasmonic nanobubble-enhanced endosomal escape processes for selective and guided intracellular delivery of chemotherapy to drug-resistant cancer cells. Biomaterials, 33(6):1821-1826, 2012.

[156] C. Bing, Y. Hong, C. Hernandez, M. Rich, B. Cheng, I. Munaweera, D. Szczepanski, Y. Xi, M. Bolding, A. A. Exner, and R. Chopra. Characterization of different bubble formulations for blood-brain barrier opening using a focused ultrasound system with acoustic feedback control. Scientific Reports, 8(1):1-12, 2018.

[157] Z. Xing, J. Wang, H. Ke, B. Zhao, X. Yue, Z. Dai, and J. Liu. The fabrication of novel nanobubble ultrasound contrast agent for potential tumor imaging. Nanotechnology, 21(14), 2010.

[158] S. K. Hobbs, W. L. Monsky, F. Yuan, W. G. Roberts, L. Griffith, V. P. Torchilin, and R. K. Jain. Regulation of transport pathways in tumor vessels: Role of tumor type and microenvironment. Proceedings of the National Academy of Sciences, 95(8):4607-4612, 1998.

[159] H. Wu, N. G. Rognin, T. M. Krupka, L. Solorio, H. Yoshiara, G. Guenette, C. Sanders, N. Kamiyama, and A. A. Exner. Acoustic characterization and pharmacokinetic analyses of new nanobubble ultrasound contrast agents. Ultrasound in Medicine and Biology, 39(11):2137-2146, 2013.

[160] R. H. Perera, H. Wu, P. Peiris, C. Hernandez, A. Burke, H. Zhang, and A. A. Exner. Improving performance of nanoscale ultrasound contrast agents using N,N-diethylacrylamide stabilization. Nanomedicine: Nanotechnology, Biology, and Medicine, 13(1):59-67, 2017. 

BIBLIOGRAPHY 
